\title{
BRIDGING BIOCHEMICAL ACTIVITIES WITH CONFORMATIONAL DYNAMICS OBSERVED IN ATOMIC FORCE MICROSCOPY
}

\author{
A Dissertation \\ presented to \\ the Faculty of the Graduate School \\ at the University of Missouri-Columbia
}

In Partial Fulfillment

of the Requirements for the Degree

Doctor of Philosophy

by

KANOKPORN CHATTRAKUN

Dr. Gavin M. King, Dissertation Supervisor

MAY 2020 
The undersigned, appointed by the dean of the Graduate School, have examined the dissertation entitled

\title{
BRIDGING BIOCHEMICAL ACTIVITIES WITH CONFORMATIONAL DYNAMICS OBSERVED IN ATOMIC FORCE MICROSCOPY
}

\author{
presented by Kanokporn Chattrakun, \\ a candidate for the degree of doctor of philosophy, \\ and hereby certify that, in their opinion, it is worthy of acceptance.
}

Dr. Gavin M. King

Dr. Linda L. Randall

Dr. Ioan Kosztin

Dr. Suchi Guha 


\section{ACKNOWLEDGEMENTS}

I would like to acknowledge my advisor, Dr. Gavin King, who had always provided a tremendous amount of support and guidance throughout my years as a graduate student at the University of Missouri. He is definitely the person that I would go to if I had any questions or problems. I am so thankful for his careful attention and his patience. I am grateful that he always provided his professional opinions while leaving space to cultivate imagination in my own research. Thank you for letting me be creative and allowimg me to do what I do.

I would also like to thank Dr. Linda Randall and her lab members who not only supplied me with samples for my research, but also valuable advice and training. I am especially thankful that I was given an opportunity to work with Lin. She is an exemplary role model for how important doing research really is. I would also like to acknowledge Dr. Chunfeng Mao for her patience in teaching me, a physicist, to do biochemical lab work. She also provided excellent opinion and discussions throughout my entire project. Without their guidance, I would have been lost and this project would never have come to this level of fruition.

I definitely would like to thank the members of the King lab: Dr. Krishna Sigdel, Dr. Anna Pittman, Dr. Nagaragu Chada, Dr. Raghavendar Reddy Sanganna Gari, Dr. Tina Matin, Katie Schaefer, Brendan Marsh, and Lucas Chandler. I cannot believe that I had the privilege to get to know so many awesome people. Thank you for your trainings, your discussions, your companionships, and your support.

Lastly, I would like to thank the Physics and Astronomy department who saw potential in me and took me in as a trainee for the program. I would also like to thank them for their effort of providing a resourceful environment for an international student like me to be able to flourish. 


\section{TABLE OF CONTENTS}

\section{Contents}

Acknowledgements $\quad$ ii

List of Illustrations vi vi

Abstract $\quad$ xi

1 Introduction 1

1.1 Atomic Force Microscopy . . . . . . . . . . . . . . . . . . . . . 1

1.2 General Secretory System $(\mathrm{Sec}) \ldots \ldots \ldots$

1.3 Activities of surface adsorbed proteins . . . . . . . . . . . . . 10

1.4 Neutron Reflectometry . . . . . . . . . . . . . . . . . . . . . 12

2 ATP hydrolysis activities $\quad 16$

2.1 Basal ATP hydrolysis . . . . . . . . . . . . . . . . . 17

2.2 Translocase Activated ATP hydrolysis . . . . . . . . . . . . . 20

2.3 Translocation Associated ATP hydrolysis . . . . . . . . . . . . . 23

3 Translocation activities $\quad 29$

3.1 Surface translocation activities assay . . . . . . . . . . . . . 29

3.2 Chemo-mechanical coupling . . . . . . . . . . . . . 37

4 Submembrane characterization via Neutron Reflectometry 41

5 Membrane surface characterization via Atomic Force Microscopy 49

5.1 Single molecule observation of nucleotide induced conformational changes in basal SecA-ATP hydrolysis . . . . . . . . . . . . . . . . . 49 
5.2 Direct visualization of the E. coli Sec translocase engaging precursor proteins in lipid bilayers . . . . . . . . . . . . . . . 54

6 Additional Projects $\quad 61$

6.1 Force spectroscopy of SecA2-11 with Focused Ion Beam (FIB) modified AFM cantilever ..................... 61

6.2 Investigation of pyrophosphatase enzymes using AFM . . . . . . . . 67

$\begin{array}{lll}7 & \text { Preliminary results and future directions } & 79\end{array}$

8 Conclusions $\quad 92$

$\begin{array}{lll}\text { Appendix A On-surface activity assays } & 94\end{array}$

A.1 On-surface basal ATP hydrolysis activity . . . . . . . . . . . . . 94

A.2 On-surface translocase activated ATP hydrolysis activity . . . . . . 96

A.3 On-surface translocation associated ATP hydrolysis activity . . . . . 97

A.4 On-surface translocation activity . . . . . . . . . . . . 98

A.5 Preparation of $\mathrm{KOH}$ treated glass cover slips . . . . . . . . . . . 100

$\begin{array}{lll}\text { Appendix B In-solution activity assays } & 101\end{array}$

B.1 In-Solution ATP hydrolysis . . . . . . . . . . . . . . . . . . . 101

B.2 In-Solution translocation activity . . . . . . . . . . . 103

$\begin{array}{lll}\text { Appendix C Neutron Reflectometry } & 103\end{array}$

$\begin{array}{ll}\text { Appendix D Biochemical Methods } & 105\end{array}$

D.1 Purification of Sec Components . . . . . . . . . . . . . 105

D.2 Preparation of radiolabeled precursors . . . . . . . . . . 106

D.3 Preparation of proteoliposomes . . . . . . . . . . . . 106 
D.4 Translocation assay in solution . . . . . . . . . . . . . . 107

D.5 Liposome preparation . . . . . . . . . . . . . . . . 109

D.6 Quantification of lipid in liposome . . . . . . . . . . . . 111

D.7 Taussky-Shorr assay . . . . . . . . . . . . . . . . . . . . 113

$\begin{array}{ll}\text { References } & 116\end{array}$

$\begin{array}{ll}\text { Vita } & 127\end{array}$ 


\section{List of Illustrations}

\section{List of Figures}

1 AFM schematic . . . . . . . . . . . . . . . 1

2 Tip-sample convolution . . . . . . . . . . . . . . . 5

3 General Secretory System of E. coli . . . . . . . . . . . . . . . . . 9

4 Diagram of the specular reflection of a plane wave . . . . . . . . 13

5 Reflectivity as a function of wavevector transfer . . . . . . . . . . . 14

$6 \quad$ Neutron reflectometer geometry ................ 15

$7 \quad$ ATP hydrolysis reaction . . . . . . . . . . . . . 16

8 Basal ATP hydrolysis assay . . . . . . . . . . . . . . . 17

9 Basal ATP hydrolysis results f. . . . . . . . . . . . . . . 18

10 AFM image of SecA on mica surface . . . . . . . . . . . . . . 19

11 Crystal structure of SecA . . . . . . . . . . . . . 20

12 Translocase Activated ATP hydrolysis assay . . . . . . . . . . . . 21

13 Translocase Activated ATP hydrolysis results . . . . . . . . . . . 22

14 AFM image of translocase complexes on mica surface . . . . . . . . 23

15 Translocation Associated ATP hydrolysis assay . . . . . . . . . . . . 24

16 Translocation Associated ATP hydrolysis results . . . . . . . . . . 25

17 Comparison of translocation associated ATP hydrolysis assay on glass and mica surfaces . . . . . . . . . . . . . . . . . . . . 27

18 Translocation associated ATP hydrolysis assay on glass surface with

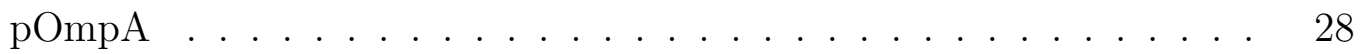

19 Surface translocation assay . . . . . . . . . . . . . . . . . . . 29

20 Surface translocation activity on glass surface . . . . . . . . . . . . 30 
21 Bar graphs indicate hallmark of translocation activity on glass surface

22 Surface translocation activity on mica surface . . . . . . . . . . . 31

23 Standard curve . . . . . . . . . . . . . . . . . . . . . 32

24 Translocation activity quantification . . . . . . . . . . . . 32

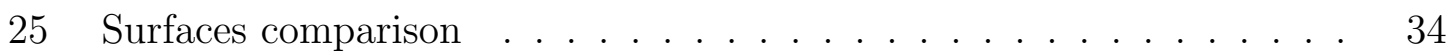

26 Control experiments . . . . . . . . . . . . . . . 35

27 ATP-related changes in lipid bilayer defects using AFM . . . . . . . . 36

28 In-solution translocation activity f . . . . . . . . . . . 37

29 Surface topography and roughness comparison between $\mathrm{SiO}_{x}$ and borosilicate glass surfaces . . . . . . . . . . . . . . . . . . 42

30 Neutron reflectivity of proteoliposomes Y·A on $\mathrm{SiO}_{x}$ surfaces . . . . . 44

31 Submembrane structure of proteoliposomes Y.A on $\mathrm{SiO}_{x}$ via NR . . . 47

32 Conformation of SecA observing using AFM . . . . . . . . . . 50

33 Height distribution of SecA-WT and SecA $\triangle \mathrm{PBD} \ldots \ldots . . . . . .51$

34 Reversible conformational dynamics of individual SecA during ATP hydrolysis. . . . . . . . . . . . . . . . . . . 53

35 Sec translocase topography varies as a function of precursor species and translocation stage. . . . . . . . . . . . . . . . 57

36 Height distribution of translocase complexes engaing in translocating precursor protein ...................... 58

37 Bayesian criteria used to determine number of model to fit the height distributions . . . . . . . . . . . . . . . . . . 59

38 SEM image of the cantilever before and after the FIB modification . . 62

39 The force power spectral density . . . . . . . . . . . . 63

40 Integrated force noise of the modified cantilever . . . . . . . . . . . 64

41 Rupture force distribution of SecA2-11 peptide and POPC lipid . . . 65 
42 Rupture force distribution obtained from the modified cantilever . . . 67

43 Emergence of multi-state of SecA2-11 peptide and POPC lipid . . . . 67

44 Standard curve of the activity of the sgPP enzyme . . . . . . . . . 69

45 Activity curves of pyrophosphatase enzymes on the surface . . . . . . 71

46 AFM simulation image of the pyrophosphatase enzyme . . . . . . . 72

47 AFM pyrophosphatase enzymes . . . . . . . . . . . . . . 73

48 Volume histograms of pyrophosphatase enzymes . . . . . . . . . . . . 74

49 Height and area distribution of pyrophosphatase enzymes . . . . . . . 75

50 Height and area distribution of tandem dimer mutated pyrophosphatase

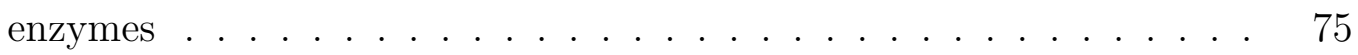

51 AFM image of pyrophosphatase enzymes with substrate activated in solution . . . . . . . . . . . . . . . . 76

52 Height and area distribution of the wild type enzyme with different pre-incubation times . . . . . . . . . . . . . . . . 77

53 Height, area, and volume distributions of the wild type enzyme with different pre-incubation times . . . . . . . . . . . . . . . 78

54 Translocase on glass surface in the presence of different nucleotide species 80

55 Height distribution of translocases in different nucleotides species . . 80

56 Area distribution of translocases in different nucleotides species . . . 81

57 Kymographs of translocase in the presence of ATP . . . . . . . . . . 82

58 Kymographs of $\mathrm{Y} \cdot \mathrm{A} \Delta \mathrm{PBD} \ldots \ldots \ldots \ldots$

59 Representative AFM image of translocase undergoing translocation on glass surface . . . . . . . . . . . . . . . . . . . 84

60 Representative kymographs of translocase undergoing translocation on glass surface . . . . . . . . . . . . . . . . . . 85

61 Kymograph analysis .................. 86 
62 Height histograms extracted from kymograph . . . . . . . . . . 86

63 Height distributions of active translocases translocating pOmpA . . . 88

64 Height distributions of translocases undergoing pOmpA translocation over time. . . . . . . . . . . . . . . . . . . . . . . . 89

65 Fraction of SecYEG and SecA over time. . . . . . . . . . . . . . 90

$66 \quad \mathrm{Y} \cdot \mathrm{A}$ translocation gel quantification . . . . . . . . . . . . . . 108

67 Gel quantification of protein in proteoliposomes . . . . . . . . . . 108

68 Liposome extrusion . . . . . . . . . . . . . . . . . 111

69 Stand and Sample concentration curve for lipid concentration quantifi-

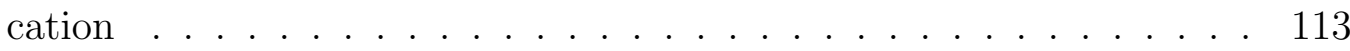




\section{List of Tables}

1 Comparing surface ATP hydrolysis assays to solution assays . . . . . 26

2 Comparing chemo-mechanical coupling of surface translocation assays and in solution assays . . . . . . . . . . . . . . . . . . 38

3 Neutron reflectometry fitting data . . . . . . . . . . . . . . . 48

4 Total phosphorous assay . . . . . . . . . . . . . . . . . 112

5 Taussky-Shorr assay . . . . . . . . . . . . . . . . . . 114 


\section{Abstract}

This dissertation was written with the intention to provide future investigators with general information sufficient to start their own investigations in biological atomic force microscopy. It is noted that background information from both physics and biology has been included for overall clarity. A main emphasis of my thesis work was on modifying traditional assays to measure biochemical activities of membrane proteins adsorbed on surfaces prepared in an identical manner for atomic force microscopy (AFM) measurements. Additional projects included probing conformational dynamics of enzymes and utilizing atomic force spectroscopy to probe peptide-lipid interactions at enhanced temporal resolution via focused ion beam (FIB) modified AFM cantilevers. The experimental procedures in the appendix were purposefully written in a step by step format, with detailed notes of important or tricky aspects and precautions. Thus, these sections could serve as practical templates to construct future protocols and experiments. A chapter on future directions serves as suggestions of possible avenues of research.

AFM measurements can shed light on membrane protein conformational dynamics and folding at a single molecule level. However, the unavoidable close proximity of the supporting surface to AFM specimens raises questions about the viability and preservation of biochemical activities. We quantified activities of the translocase from the general secretory (Sec) system of Escherichia coli, (E. coli), via two biochemical assays in surface supported bilayers: ATP hydrolysis and translocation. The ATP hydrolysis assays revealed distinct levels of activation ranging from low (basal), to medium (translocase-activated), to high (translocation-associated) corresponding to binding partners of SecA, the ATPase enzyme that hydrolyzes ATP. The measured onsurface ATP hydrolysis activity levels were similar to traditional solution experiments. Furthermore, the surface activity assays uncovered characteristics of conformational 
hysteresis of SecA. Translocation assays displayed turn over numbers that were comparable to solution but with a reduction in the apparent rate constant. Despite a 10-fold difference in kinetics, the chemomechanical coupling (ATP hydrolyzed per residue translocated) only varied twofold on glass compared to solution. The activity changed with the topography of the supporting surface underneath the lipid bilayer. Glass cover slips have higher surface roughness than that of mica; this roughness can provide extra submembrane space. In turn, this extra space could lower the frictional coupling between the translocating polypeptide and the supporting surface. For these reasons, glass surfaces were favored over mica. Neutron reflectometry corroborated the results and provided characterization of the integral and peripheral components, as well as the submembrane space between the surface and the lower bilayer leaflet. Overall, surface activity assays had sufficient sensitivity to distinguish different levels of ATP hydrolysis and translocation activities of surface adsorbed systems, albeit with a slower rate-limiting step than observed in solution assays.

Equipped with biochemical activity information for the surface-adsorbed proteins, we could then more strongly correlate conformational dynamics of the proteins observed in AFM measurements to their biochemical activities. We conducted AFM investigations on conformational dynamics of SecA on mica surfaces yielding fruitful information to specify the domain responsible for conformational dynamics during the ATP hydrolysis cycle. We also investigated the dynamics of translocase complexes engaging in translocation of precursor proteins across the membrane surface. These experiments brought to light previously underappreciated precursor species dependent conformational dynamics of the translocase. 


\section{Introduction}

\subsection{Atomic Force Microscopy}

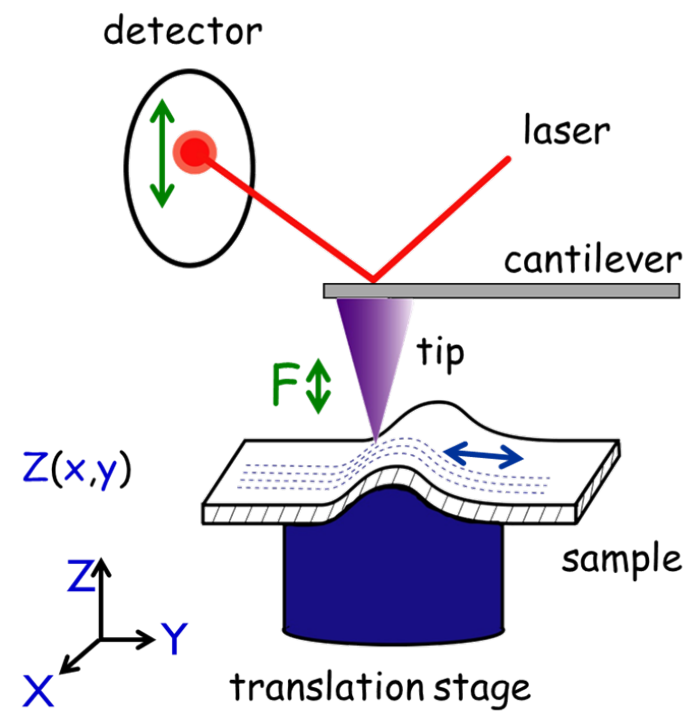

Figure 1: AFM schematic. The schematic shows components of an atomic force microscope

AFM is a member of a larger group of instruments categorized generally as Scanning Probe Microscopes (SPMs). Their function is based primarily on the strong distance dependent interaction between a sharp probe or tip and a sample. Since AFM can be operated in aqueous solution, it has opened an avenue for use in biology [1]. The data obtained by an AFM is essentially transduced through sensing with a probe. The crucial part of AFM is therefore the apparatus that performs this sensing which is the cantilever with a nanometer scale tip. A laser is then focused onto the back side of the cantilever to keep track of the movement of the cantilever, where a quadrant photodiode is used to detect the laser signal. A sample is placed on a translation stage which can move in the three dimensions: $\mathrm{x}, \mathrm{y}$, and $\mathrm{z}(\mathrm{x}, \mathrm{y})$. Figure 1 shows the schematic of a typical AFM. 
Piezoelectric devices are used to accurately control the position of both the tip and the sample. Because typical bonding force constants in a crystalline lattice are on the order of $\sim 1 \mathrm{~N} / \mathrm{m}$, the spring constant of a cantilever should be about the same value, which is equal to $\sim 1000 \mathrm{pN} / \mathrm{nm}$. To achieve this value, the cantilever must be of a micrometer scale because $k \sim \frac{E t^{3} w}{4 l^{3}} ; k$ is the spring constant, $E$ is Young's modulus of the cantilever, $t$ is the thickness of the cantilever, $w$ is the width of the cantilever, and $l$ is the length of the cantilever. Since the behavior of the cantilever can be explained using physical behavior of a spring, the limit in measuring distance using a cantilever operated as a spring is governed by the energy of oscillation and thermal energy. $\frac{1}{2} k\left\langle\Delta x^{2}\right\rangle=\frac{1}{2} k_{B} T$. Therefore, thermal fluctuations lead to a deviation in measuring distance of $\Delta x=\sqrt{\frac{k_{B} T}{k}}$, where $k_{B}$ is a Boltzmann constant and $T$ is a temperature in Kelvin. For a cantilever with $k \sim 0.1 \mathrm{~N} / \mathrm{m}$ or $100 \mathrm{pN} / \mathrm{nm}$, the thermal noise is $\sim 0.2 \mathrm{~nm}$ or $2 \AA$, which is approximately the atomic corrugation of a surface. Therefore, AFM can be used as a tool to study atomic scale structures. Although scale on the order of $\AA$ is fundamentally difficult to achieve by an AFM, atomic lattices and molecular resolution imaging is achievable and can provide useful information about the dynamics of a system [2-9]. The stiffness of the cantilever, $k$, is proportional to its resonance frequency which is very important when operating an AFM in imaging using tapping mode, because this is a parameter that determines the imaging speed. $f=\frac{1}{2 \pi} \sqrt{\frac{k}{m}}=\frac{1}{4 \pi} \frac{t}{l^{2}} \sqrt{\frac{E}{\rho}}, f$ is the resonance frequency of the cantilever, $m$ is the mass of the cantilever, and $\rho$ is the density of the cantilever material. In order to respond to surface corrugation that has characteristic wavelength on the order of $\mathrm{nm}$ (the dimension of an average protein) and maintain reasonable imaging speeds, the cantilever should have a resonance frequency $>\mathrm{kHz}$. Therefore, a soft (low $k$ ) cantilever operated at high resonance frequency is advantageous for fast imaging of soft materials. State of the art high speed AFM cantilevers approach $1 \mathrm{MHz}$ reso- 
nance frequencies in fluid by minimizing the mass, $m[10,11]$.

Imaging in solution has a different set of challenges compared to more common imaging mediums (air or vacuum). In solution, the charge of an object is typically screened by the ions in the solution. This has impact on the interactions experienced by the cantilever. Electrostatic interactions play a central role in tip-surface interactions, and hence, the underlying image resolution. Fine tuning the concentration of ions in the buffer is required for high resolution imaging in solution as well as a cantilever that is soft, yet with a high resonance frequency. Increasing the ionic strength of the medium suppresses long range electrostatic interactions between the tip and protein sample. The damping regime is another parameter to consider when analyzing data from force spectroscopy. To minimize the perturbation imparted upon the sample by the measurement, the cantilever used should be overdamped. The damping regime depends on both the geometry and the viscosity of the fluid environment. The motion of the cantilever in a viscous fluid environment is governed by

$$
m \frac{d^{2}}{d t^{2}} x(t)+\beta \frac{d}{d t} x(t)+k x(t)=F(t)
$$

$\beta$ is the hydrodynamic drag or the damping coefficient of the motion. When the damping is large $\beta^{2}>4 m k$, the motion is overdamped, which is the regime preferable for operating atomic force microscope in fluid environment. It is possible to solve equation 1 , assuming the form of force is known. One can also solve equation 1 using Laplace transform and obtain a solution that contains $x(t)=x_{\text {transient }}(t)+x_{\text {steadystate }}(t)$. The transient term is a function of initial conditions. Generally, initial conditions are $\mathrm{x}(0)$ $=0$ and $\frac{d x}{d t}=0$ at time $\mathrm{t}=0$. The steady state term depends on the force $[12,13]$. The applied force can be written in different forms depending on the situation relevant in the measurement, for example, sinusoidal driving functions are common. Alterna- 
tively, if the thermal agitation due to collisions with surrounding solvent molecules is considered, such "thermal force" is stochastic. Despite the random nature of the thermal force, a description of the statistical properties of the resulting motion can be described by the autocorrelation function, $R_{x}(\tau)$. The autocorrelation function is the correlation of the position of the cantilever at time $\mathrm{t}$ and a later time $\tau$. It is defined by

$$
\left.R_{x}(\tau)=\langle x(t) \cdot x(t-\tau)\rangle \equiv \lim _{T \rightarrow \infty}\left(\frac{1}{T} \int_{-T / 2}^{T / 2} x(t) x(t-\tau) \cdot d t\right)\right)
$$

One crucial property of the autocorrelation function is that it satisfies the equation of motion

$$
m \frac{d^{2}}{d t^{2}} R_{x}(\tau)+\beta \frac{d}{d t} R_{x}(\tau)+k R_{x}(\tau)=0, \quad \tau>0
$$

Moreover, the autocorrelation also has a maximum value when $\tau=0$, and is zero when $\tau=\infty$. This means that the autocorrelation function has the same form as the response of the cantilever to an impulsive external force. The solution to equation 3 is the sum of two exponential functions. One is fast with small amplitude, and time constant $\tau_{1}=\frac{m}{\beta}$. Another one is slow with large amplitude, and time constant $\tau_{2}=\frac{\beta}{k}$. Under a highly damped regime, $\tau_{2}>>\tau_{1}$ and $\tau_{1}$ can be neglected. Therefore, the time constant $\tau$ refers to $\frac{\beta}{k}$. Besides time constant $\tau$, quality factor $\mathrm{Q}$ is another parameter characterizing damping regime. Q factor is defined as the number of oscillations required for a freely oscillating system's energy to fall off by a factor of $535\left(\frac{1}{535}\right)$. The factor originates from $\mathrm{e}^{2 \pi}$, or about $0.2 \%$, of its original energy [14]. $Q=\frac{\omega_{0} \tau}{2}$, where $\omega_{0}$ is a natural frequency of oscillation measured prior to experiment in the absence of damping. Note that the frequency of oscillation changes in the presence of damping force. Operating in the overdamped regime is preferred since it helps increase the precision of the AFM measurement [15] by minimizing inadvertent driving forces at 
the cantilever resonance frequency. For the damping to be "overdamped", Q must be much less than $0.5[10,16]$. One more beauty of the autocorrelation function is that it appears in the power spectral density. Indeed, the Fourier transform of the autocorrelation function is called the power spectrum, $G_{x}(f)$. It is defined so that $G_{x}(f) \cdot \Delta f$ is the mean square value of the signal $R_{x}(\tau)$ after passing through a notch filter with a bandwidth of $\Delta f$. The power spectrum or power spectral density curves are used routinely to characterize AFM cantilevers by revealing the damping regime and resonance frequency of the cantilever.

Ultimately, image resolution is limited by the geometry of the tip. The finite dimension of the tip produces images of objects that appear wider than they really are by convolution. This is especially relevant for imaging a biological sample where dimensions are smaller than that of the AFM tip. Figure 2 depicts a cartoon explanation on the effect of AFM tip convolution to the dimension of the sample imaged.
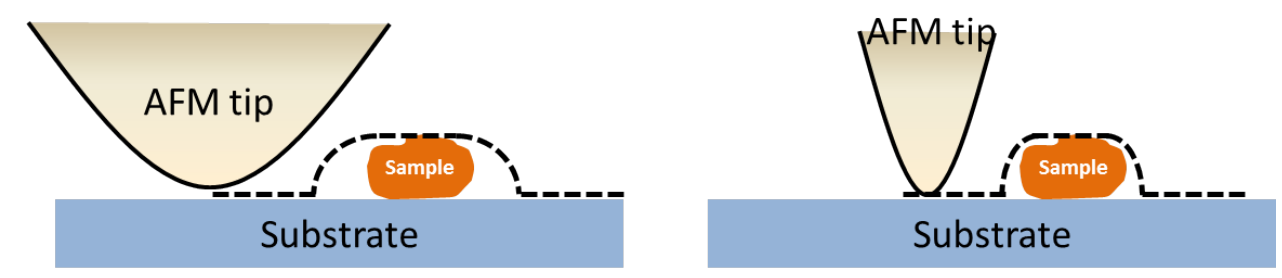

Figure 2: AFM tip-sample convolution effect in imaging

The shape of the tip can also introduce artifacts in acquired images. For instance, a common defect of tip fabrication results in two apexes which leads to images that appear to be replicated, separated by a distance equal to the distance between the two apexes. The feedback loop also plays a critical role in creating artifacts introduced in images, for example by ringing. Thus, additional care must be implemented when taking images in fluid.

Surface adsorbed biomolecules immersed in a buffer solution containing charged 
particles develop a surface charge creating an electrical double layer (EDL). When the surface and tip approach each other, the EDL's of each overlap. The force of the interaction between the sample and the AFM tip can be measured. Van der Waals and electrostatic forces are the two major forces that play important roles in the interaction between tip and sample. Electrostatic force arises from the acidic and basic functional groups of the macromolecules in aqueous environment. The Poisson-Boltzmann equation leads to the exponentially decay of the counterion concentration with the distance from a charged surface. The exponential decrease of the electric field within the solution is given by the Debye length, $\lambda_{D}$, which is also known as EDL [17]. Forces exerted by the cantilever can be recorded and used to interpret interactions between protein-protein, protein-lipid or protein folding and unfolding $[16,18,19]$. This mode of AFM operation is called force spectroscopy, as mentioned briefly above. In this mode of operation, instead of driving the cantilever in a raster fashion, the cantilever is driven toward and away from the surface at a rate that is much slower than the resonance frequency of the cantilever. The spring constant of an individual cantilever is thus important and must be determined beforehand with precision [20]. Single molecule force spectroscopy, SMFS, directly measures the behavior of one molecule at a time. It serves as a platform to understand the interaction of biomolecules by recognizing the heterogeneities inherent in each of the biomolecules studied, which traditional bulk measurements cannot easily achieve. Another advantage of SMFS is its ability to probe the biological process in real time within a near native environment. Since the tip and cantilever used strongly influence the outcome of the experiment, the choice of probe to be used must be carried out with thorough consideration.

Enzymes represent one very interesting and large class of biomolecules. Enzymes are macromolecules in biology that accelerate chemical reactions forming a product 
molecule from a substrate molecule. Note, the word substrate here refers to a small molecule that the enzyme converts into another small molecule (i.e. a product), and not a specimen supporting surface. The enzyme accelerates the reaction by reducing the activation energy or the energy barrier of the reaction [21,22]. Most chemical reactions that occur in living cells require enzymes to increase the rate of the reaction to achieve sufficient amount of product for normal cell function. Enzymes true function occurs when a substrate binds the enzyme at its active site. The binding is usually noncovalent in nature, such as hydrogen bonding, ionic or hydrophobic interactions, but it is usually specific, governed by the detailed local surface chemistry, topography, and electrostatics. Only substrates that are specific to the enzyme's binding site can bind with the enzyme and accelerate the conversion of the substrate to the product. There are many models proposed to explain the enzyme-substrate binding; lock and key model, and induced fit, for example [23]. The lock and key model suggests that the substrate fits precisely to the active site or binding site of the enzyme like a key for a specific lock. Substrates of different shapes and sizes do not fit within the enzyme's binding site, and therefore the interaction is specific to that particular substrate. Other models such as the induced fit model suggest that the substrate can induce distortion in the local enzyme structure resulting in conformational changes of the enzyme. The changes thus help the substrate to fit within the enzyme binding site, thus accelerating the interaction. The configuration changes induced by the presence of a substrate to both substrate and enzyme is compatible with the flexible nature of biological molecules such as the enzymes themselves. AFM is a well-suited tool to perform investigations of such phenomena $[9,24,25]$. 


\subsection{General Secretory System (Sec)}

Many proteins in living cells need to be transported across membranes to properly localize and function. In fact, more than $30 \%$ of newly synthesized proteins in Escherichia coli, ( E. coli) are exported across the cytoplasmic membrane to their final destinations, where they acquire natively folded and functional state [26, 27]. Different pathways have evolved to accomplish this complex task. Among protein export pathways, the general secretory system (Sec) is ubiquitous, having homologues across all domains of life. In E. coli, the general secretory system comprises a translocase consisting of SecA, a peripheral membrane adenosine triphosphatase (ATPase), in complex with a heterotrimeric, integral membrane translocon, SecYEG $[26,27]$ as shown in Figure 3. The translocon exhibits a protein conducting channel with dimensions capable of passing polypeptides through the cytoplasmic membrane. Proteins destined for the Sec pathway are produced at the ribosome as precursors with a signal sequence. The Sec system cannot translocate precursor proteins with a stable tertiary structure $[27,28]$. One role of the signal, an amino-terminal extension of the mature protein sequence, is often to allow the chaperone SecB to capture the precursor before it folds. In a highly dynamic ATP-dependent process that is only superficially under-

stood, the precursor is passed from SecB to SecA and then through the membrane via SecYEG. 


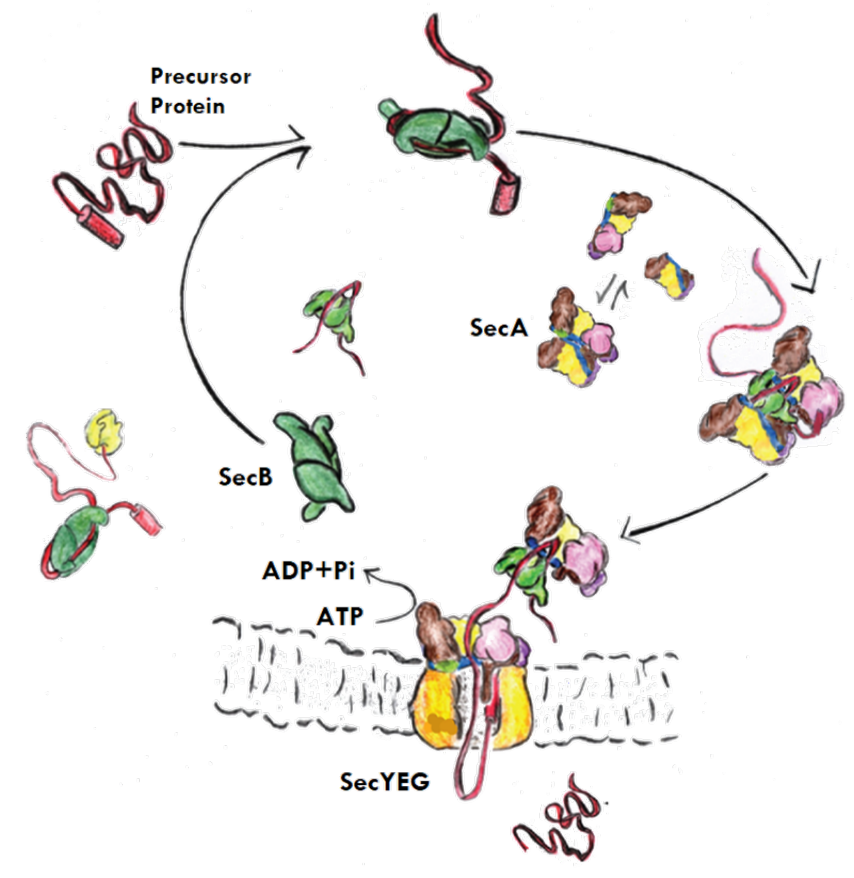

Figure 3: Key components of the Sec system in E. coli are shown. The translocon SecYEG, the ATPase SecA, and the chaperone SecB all work in concert to orchestrate the translocation of precursor proteins, the newborn protein with signal sequence still intact (as shown in the red cylinder at the amino terminal). Credit:

Illustration drawn by Dr. Linda L. Randall

Peripheral membrane protein SecA plays a central role in orchestrating translocation. Structures of SecA and other Sec system components have been obtained at high resolution and have significantly advanced the field. SecA is a large multidomain protein (901 aminoacyl residues) with numerous binding partners including the translocon SecYEG, chaperone SecB, precursor proteins, and lipids [7,29-31]. The multiple and complex functions that this macromolecule performs in a cell have likely led to the remarkable dynamics and flexibility that the domains of SecA exhibit [26, 32]. However, the inherent complexity and dynamic interplay among components of the system have left central questions unanswered, including the nature of the translocation step itself. For example, the precursor-binding domain (PBD) is thought to move approximately $80^{\circ}$ relative to other domains. Several models have been pro- 
posed to explain protein translocation with different roles for SecA, and interdomain allosteric regulation of ATPase activity is a common theme among them. SecA acts as a processive motor in a "piston" model where extended precursor protein segments move along its clamp [33,34]. The tip of the two-helix finger has been proposed to latch onto the preprotein chain and push it through the SecYEG pore. The precursor is then translocated through the channel via adenosine triphosphate (ATP) hydrolysis-driven. Evidence of power stroke, Brownian ratchet, and other mechanisms has been reported $[26,27,35,36]$. Direct observation of conformations and conformational dynamics of active translocases are made possible through single-molecule methodology, which relaxes the requirement of ensemble averaging. Though technically challenging, single-molecule investigations of the Sec system have begun in earnest and have provided insights inaccessible to traditional biochemical methods [6,24,37-44]. Aspects of the translocation reaction have been observed to vary with the precursor species undergoing transport $[7,45,46]$. However, precursor-dependent translocation mechanisms have been largely overlooked in the literature [47].

\subsection{Activities of surface adsorbed proteins}

The atomic force microscope (AFM) is a powerful single molecule tool that is capable of visualizing dynamic conformations of individual membrane proteins with $\sim 1 \AA$ vertical (normal to the membrane surface) and $\sim 1 \mathrm{~nm}$ lateral resolution [48]. AFM has been utilized to study Sec system components in isolation as well as in supported lipid bilayers [6-8,24,41,44]. The advantages which AFM provides include the ability to monitor conformational dynamics in solution without the need for additional labeling. However, the technique requires that the specimens be adsorbed to a supporting surface, though bilayer stacking, tethering, or other cushioning methods that have been developed to modulate the submembrane space [49-52]. Regardless 
of the bilayer preparation method employed, there is a need for quantitative activity benchmarks. Questions about the preservation of biochemical activity in surfacesupported systems, and hence biological relevance, often arise. For example, to make progress in understanding the Sec system via AFM or other precision surface-coupled single molecule assays [53], it is important to determine if the biochemical activity of the system is altered by proximity to the underlying solid-state surface. Additionally, once established, translocation activity can be used as a metric to quantitatively compare and to optimize supported lipid bilayer systems. Through the years there have been attempts to verify that surface-adsorbed proteins retain biochemical activity $[9,54-62]$. Early in the field of biological AFM investigation, transient deflections of cantilevers positioned above surface adsorbed lysozymes were reported to result from enzymatic turn over, though following this strategy is challenging due to low frequency instrumental noise (i.e., tip-sample drift) [9]. More recently, evidence of activity has come from direct imaging of motor proteins "walking" along cytoskeletal tracks $[59,60]$ though this approach can only be applied to a limited set of motile proteins. Hence, several methods have been used to probe the activity of surfaceadsorbed proteins, but have exhibited varying degrees of success and generality.

In my thesis work, protein translocation activity was demonstrated in surfacesupported lipid bilayers and was used in concert with ATPase assays to deduce chemomechanical coupling (ATP hydrolyzed per residue translocated) in a surfacedependent manner. Hence, two biochemical assays (ATP hydrolysis and translocation) were used to verify activity of the surface-adsorbed Sec system prepared for precision surface-coupled measurements such as AFM [24,41]. The methods were adopted from highly sensitive techniques that monitor separate aspects of the chemomechanical reaction underlying Sec system function $[27,63]$.

To probe the ATP hydrolysis activity, we tracked dephosphorylation of radioactive 
$\left[\gamma_{-}{ }^{32} \mathrm{P}\right]$ ATP. Different levels of the hydrolysis activity were found to be distinguishable with the method. Interestingly, SecA that was released from membrane-bound translocases remained in a highly activated state, rapidly hydrolyzing ATP for extended time periods, suggestive of conformational hysteresis.

To probe translocation activity, we followed the protection of radiolabeled precursor proteins from degradation by an added protease. Two surfaces were utilized: freshly cleaved mica and cleaned borosilicate glass coverslips. Glass-supported Sec translocases exhibited a substantial enhancement in translocation activity compared to mica. This may be attributable to the topographic asperities of glass providing more space for the precursor to occupy. Two translocating polypeptide species were employed, the precursor of outer membrane protein $\mathrm{A}$ (pOmpA) and the precursor of galactose-binding protein (pGBP). The data revealed that the hallmark of the Sec system activity is preserved in mica or glass supported translocases: ATP dependent precursor protection. The results of all surface-adsorbed assays were compared to assays carried out in solution wherein the translocons can be taken to be in a native environment.

\section{$1.4 \quad$ Neutron Reflectometry}

Neutron reflectometry (NR) is a powerful method to determine compositions of biological systems such as lipid bilayers and biomolecules on supporting surfaces. Of particular relevance to this work is NR's ability to access the submembrane space of a supported lipid bilayer. This capability of NR is unique as this region of space is inaccessible to many other tools such as AFM, which only probes the surfacedistal membrane region. Because the lipid bilayer is largely transparent to neutrons and neutrons are sensitive to hydrogen and deuterium, NR can complement AFM investigations and vice versa. NR profiles can be used to distinguish the structure of 
adsorbed membrane and membrane proteins $[64,65]$. More specifically, the scattering length density (SLD) depth profile can be deduced from reflectivity measurements. $\mathrm{D}_{2} \mathrm{O}$, a form of water with a deuterium isotope, is used in NR measurement to enhance contrast since it attenuates neutrons differently from normal water, $\mathrm{H}_{2} \mathrm{O}$.

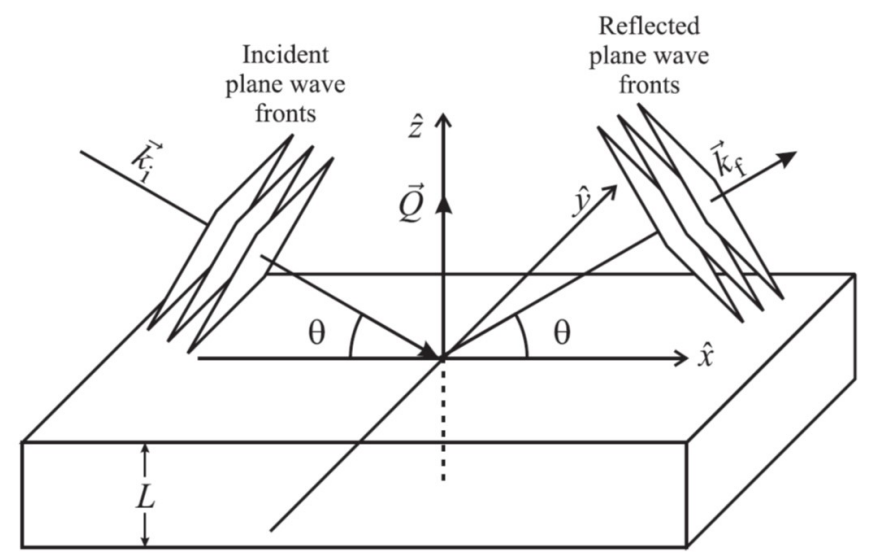

Figure 4: Diagram of the specular reflection of a plane wave from a homogeneous flat slab of thickness L (and of infinite lateral extent in the $x-y$ plane). [66]

Figure 4 portrays a diagram of the specular reflection $\left(\theta_{\text {incident }}=\theta_{\text {reflected }}\right)$ of a plane wave from a homogeneous flat slab of thickness L (and of infinite lateral extent in the $x$-y plane). The wavevector transfer, $\vec{Q}$, is defined as $\overrightarrow{k_{f}}-\overrightarrow{k_{i}}$, where $\overrightarrow{k_{f}}$ and $\overrightarrow{k_{i}}$ are wavevectors of reflected and initial wavefronts in the medium, respectively. Q is related to the angle, $\theta$, and the wavelength, $\lambda$, by $Q=\frac{4 \pi \sin \theta}{\lambda}$. SLD can be calculated from the wavevector and the refractive index of the material as shown in equation 4 .

$$
n=\sqrt{\frac{k_{0}^{2}-4 \pi \rho}{k_{0}^{2}}}
$$

$k_{0}$ is the neutron wavevector in vacuum, $n$ is the index of refraction of the medium, and $\rho$ is the scattering length density. The reflectivity is defined as reflected intensity over incident intensity denoted as $|r|^{2}$ and is measured as a function of wavevector transfer, $Q$. 


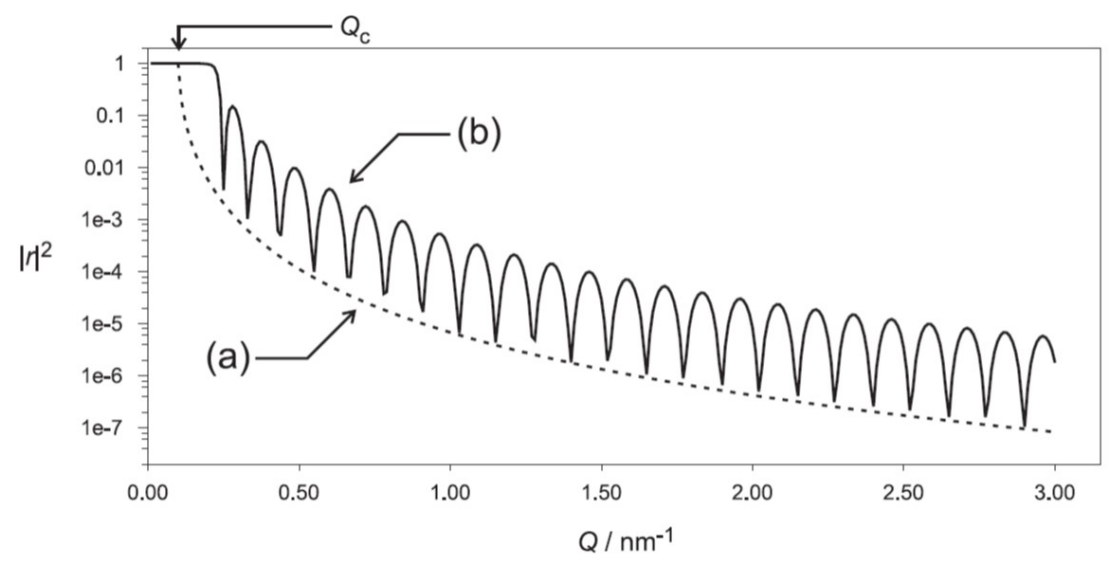

Figure 5: Reflectivity vs. Q for: a) a semi-infinite substrate of single crystalline Si; and b) a uniform $500 \AA$ thick (nonmagnetic) film of $\mathrm{Ni}$ on a semi-infinte $\mathrm{Si}$ substrate. Note the flat region of total or mirror reflection below a critical value of Q. The oscillations of the reflectivity in b) are called Kiessig fringes and result from the self-interference of the neutron waves it is simultaneously reflected from the front and back of the Ni film. [66]

Figure 5 shows the reflectivity $|r|^{2}$ versus the wavevector transfer, Q, for the two SLD profiles: a) is a profile from a perfectly smooth surface boundary, and b) is a profile from a semi-infinite substrate with a homogeneous layer deposited on its surface. The oscillations of the reflectivity curve corresponding to the layer of finite thickness shows signature fringes called Kiessig fringes, resulting from interference of reflected waves from the film interfaces (front and back). Kiessig fringes depend on the species, the thickness, the roughness of the film, etc. Therefore, such information can be deduced from the reflectivity profiles.

Figure 6 illustrates the geometry of the reflectometer used in our experiments. Incoming neutron particles from the neutron source pass through pre-sample collimators, incident on the sample's surface. The surface is mounted in a flow cell so fluid can be exchanged. The reflected neutrons pass through post-sample slits and then the detector. Care and caution must be taken to estimate and eliminate background so that the sample's profile is deduced correctly [67]. 


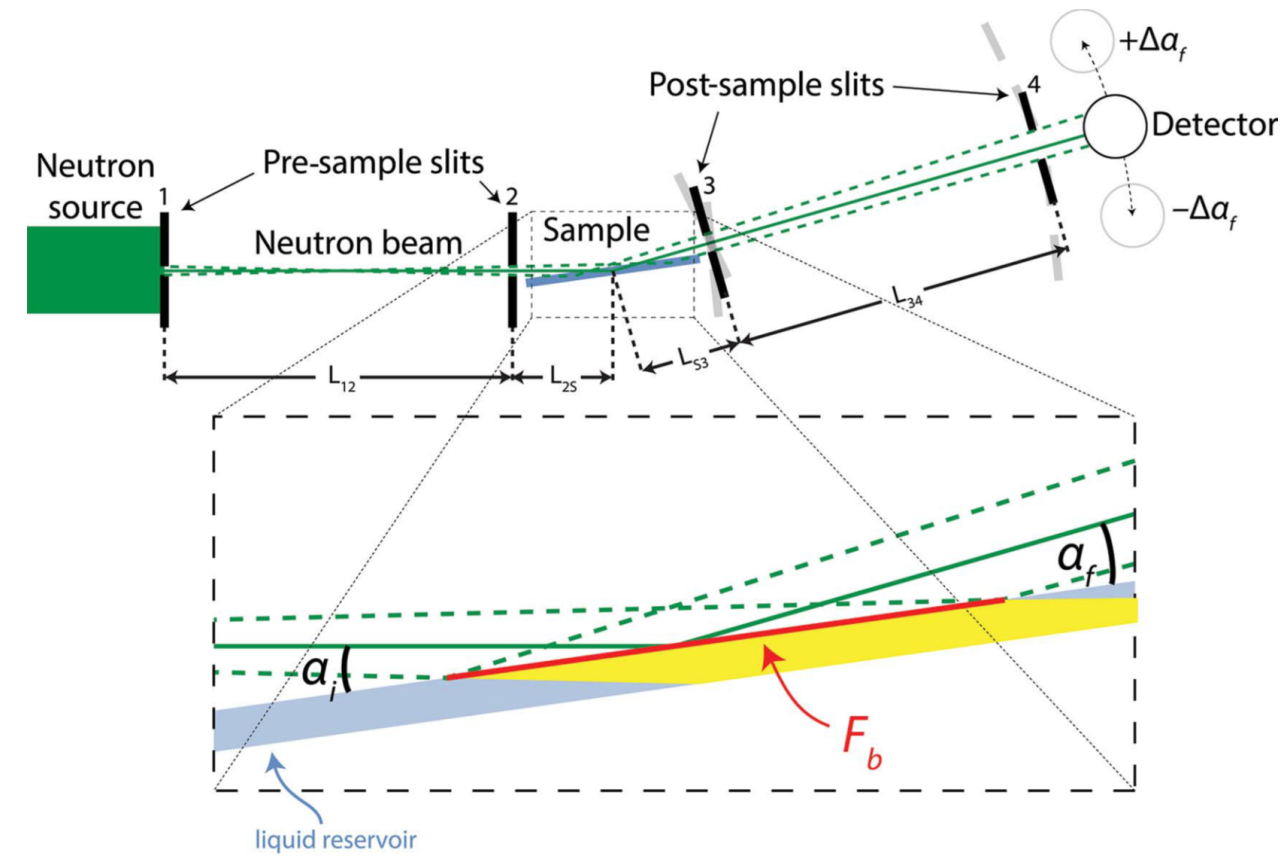

Figure 6: Collimating slits are labeled 1 through 4 in order of their position along the beam direction. Dashed lines show the maximum beam divergence. Pre-sample slit openings are chosen as a function of incident angle $\alpha_{i}$ to maintain a constant footprint $\mathrm{F}_{b}$ on the sample (expanded inset), while post-sample slit openings are chosen so as not to block any neutrons reflected from the sample when the reflection angle $\alpha_{f}$ is equal to $\alpha_{i}$, i.e. the specular reflection condition. A common method of estimating the contribution of background scattering to the measured specular reflection is linear interpolation of the background field measured at larger $(+)$ and smaller (-) detector rotations $\Delta \alpha_{f}$. [67] 


\section{ATP hydrolysis activities}

ATP hydrolysis activities assisted by SecA show distinct levels depending on the binding partner(s) present. The lowest level is called the basal level where SecA is the only component in the solution. A medium level involves SecA and SecYEG, called the translocase complex. This level is called "translocase activated" ATP hydrolysis. The highest level of ATP hydrolysis occurs when all the central components of the general secretory system: SecA, SecYEG, SecB, and precursor protein, are present. This highest level is called "translocation associated" ATP hydrolysis. There are several methods to quantify ATP hydrolysis activities. Due to the low number of surface adsorbed proteins, the detection method must have sufficient sensitivity. The most sensitive method available to us is to utilize radioactive phosphorous to trace dephosphorylation. Figure 7 depicts substitution of radioactive phosphorous into the gamma phosphate group (indicated as yellow circles in Figure 7) which is cleaved during dephosphorylation and released as an inorganic phosphate product. This inorganic phosphate product is radioactive. Thus, one can quantify ATP hydrolysis activities using standard methods.

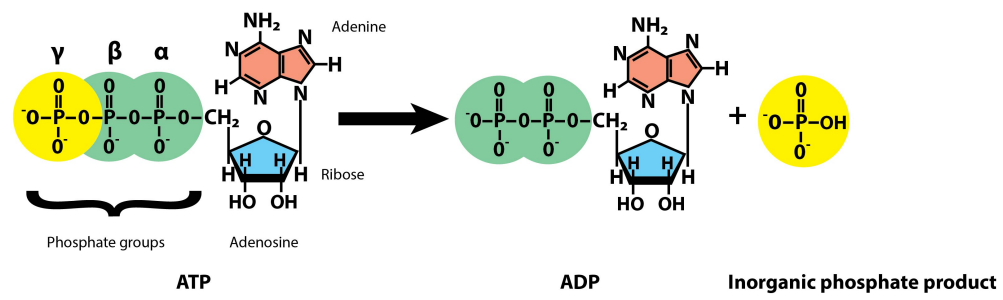

Figure 7: ${ }^{32} \mathrm{P}$ substitutes a phosphorous in the $\gamma$ phosphate group of the ATP molecule which is cleaved off during the dephosphorylation reaction. The inorganic phosphate product is radioactive, allowing the activity to be traced. 


\subsection{Basal ATP hydrolysis}

Basal ATP hydrolysis activity is the lowest ATP hydrolysis activity for the Sec system. Only SecA is a catalyst in the reaction. The experimental set up is shown in Figure 8. SecA was adsorbed on freshly cleaved mica. The surface was rinsed heavily to remove any loosely bound particles. The radiolabeled ATP was added to the surface. At determined times, the solution above the surface was sampled and mixed with reaction stop mixture containing EDTA. Thin layer chromatography was performed in phosphate based solution. The percent of ATP hydrolyzed was quantified. Please note that the experiment requires the use of radioactive material and all experiments must be handled with care. Everything that comes into contact with the radioactive material must be disposed of properly (see Appendix A.1 for a detailed experimental protocol).

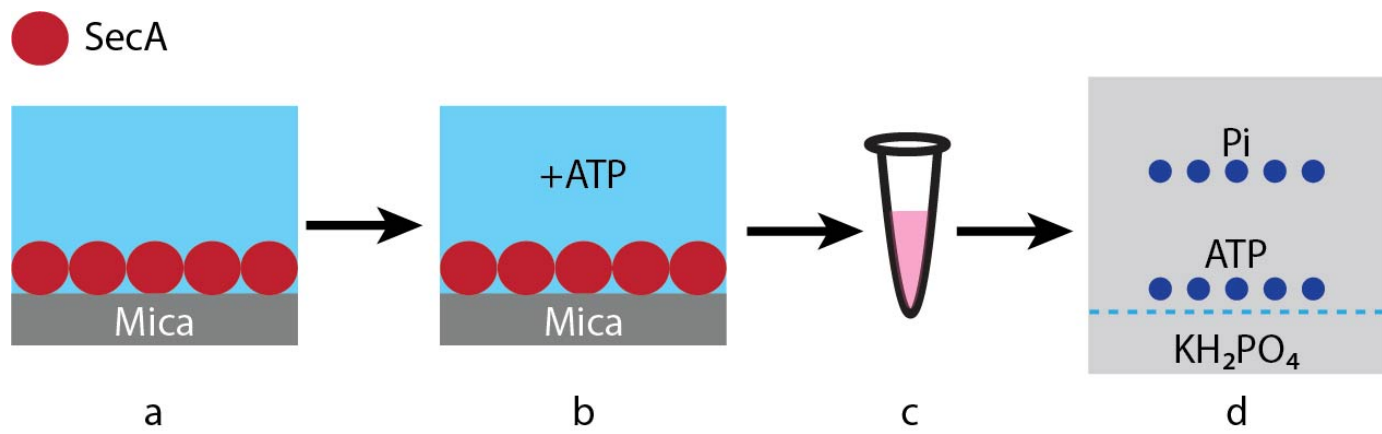

Figure 8: SecA was adsorbed on freshly cleaved mica (a). The surface was rinsed. Radiolabeled ATP was added to the surface (b). At determined times, the solution above the surface was pipetted and mixed with EDTA to terminate the hydrolysis reaction (c). Thin layer chromatography was performed in phosphate based solution (d). This plate was quantified in a phosphorimager to obtain \% ATP hydrolyzed. 


\section{Results}
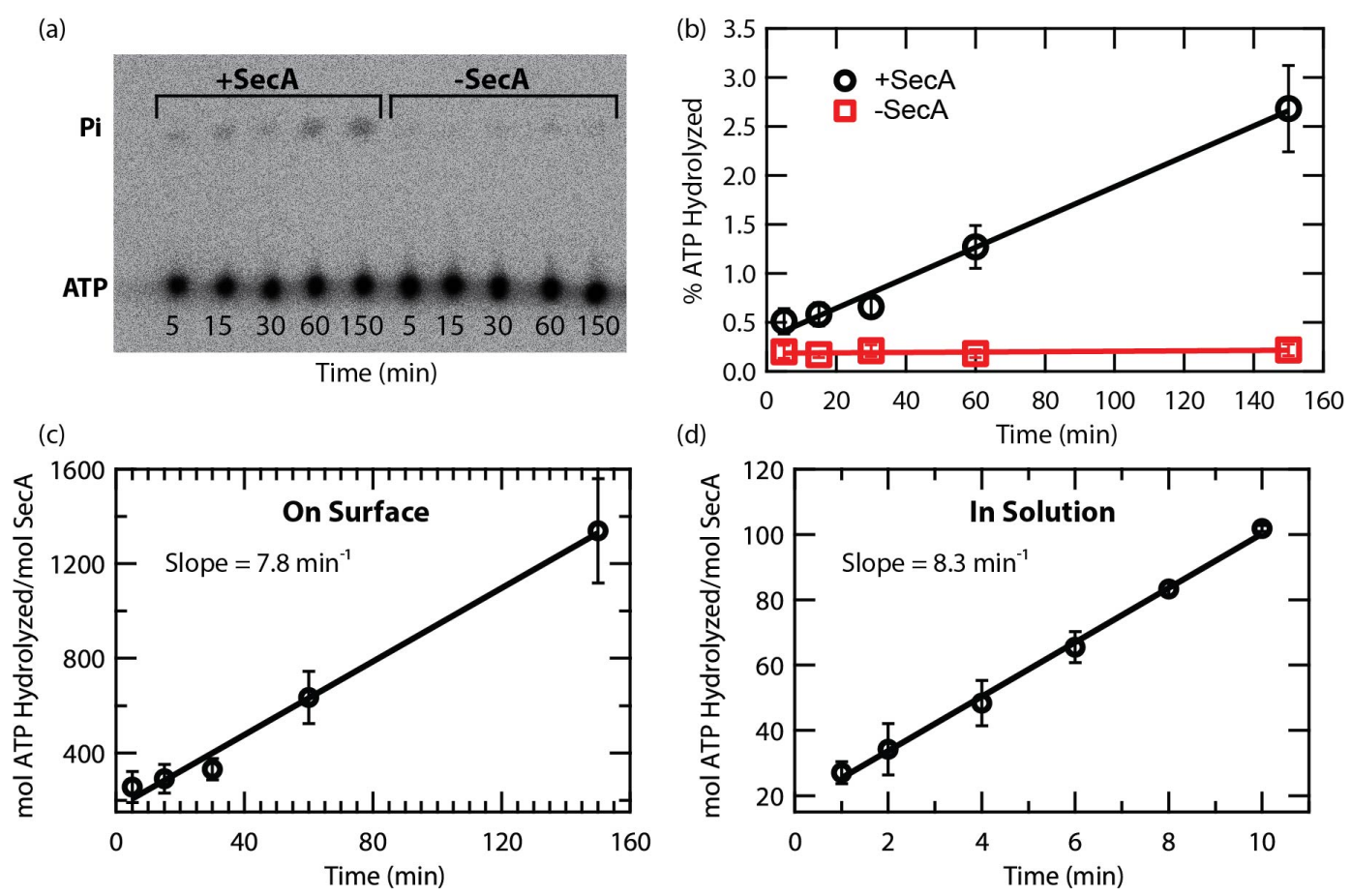

Figure 9: Surface adsorbed basal ATP hydrolysis activity and comparison with

traditional solution assay. (a) A radiograph developed from a thin layer chromatography plate showing the separation of inorganic phosphate products obtained from systems with and without SecA. (b) The \%ATP hydrolyzed as a function of time was plotted. The systems with SecA hydrolyzed ATP at a higher rate than those without, indicated by the steeper slope. (c) Mol ATP hydrolyzed was normalized with mol SecA available on the surface and plotted as a function of time. (d) Mol ATP hydrolyzed was normalized with mol SecA available in the solution and plotted as a function of time.

Figure 9a shows a radiograph developed from thin layer chromatography (TLC). The bottom row represents the original locations where the surface solutions were spotted. The top row represents the inorganic phosphate products which were smaller thus could travel further up the plate during the thin layer chromatography. After quantification, the percent ATP hydrolyzed was determined and plotted as a function of time as shown in Figure 9b. The percent ATP hydrolyzed as a function time was compared between the system with (black circles) and without (red squares) 
the presence of SecA. To make a meaningful comparison with the traditional solution assay, percent ATP hydrolyzed was converted to mol ATP hydrolyzed and normalized with mol SecA available on the surface under the assumption that the surface was completely covered with SecA. Figure 9c and 9d show mol ATP hydrolyzed/mol SecA available on the surface and in solution, respectively. Slopes of the two curves represent the rates of ATP hydrolysis. The data and analysis (discussed below) indicate that SecA adsorbed on the surface hydrolyzed ATP at a comparable rate to those in solution.

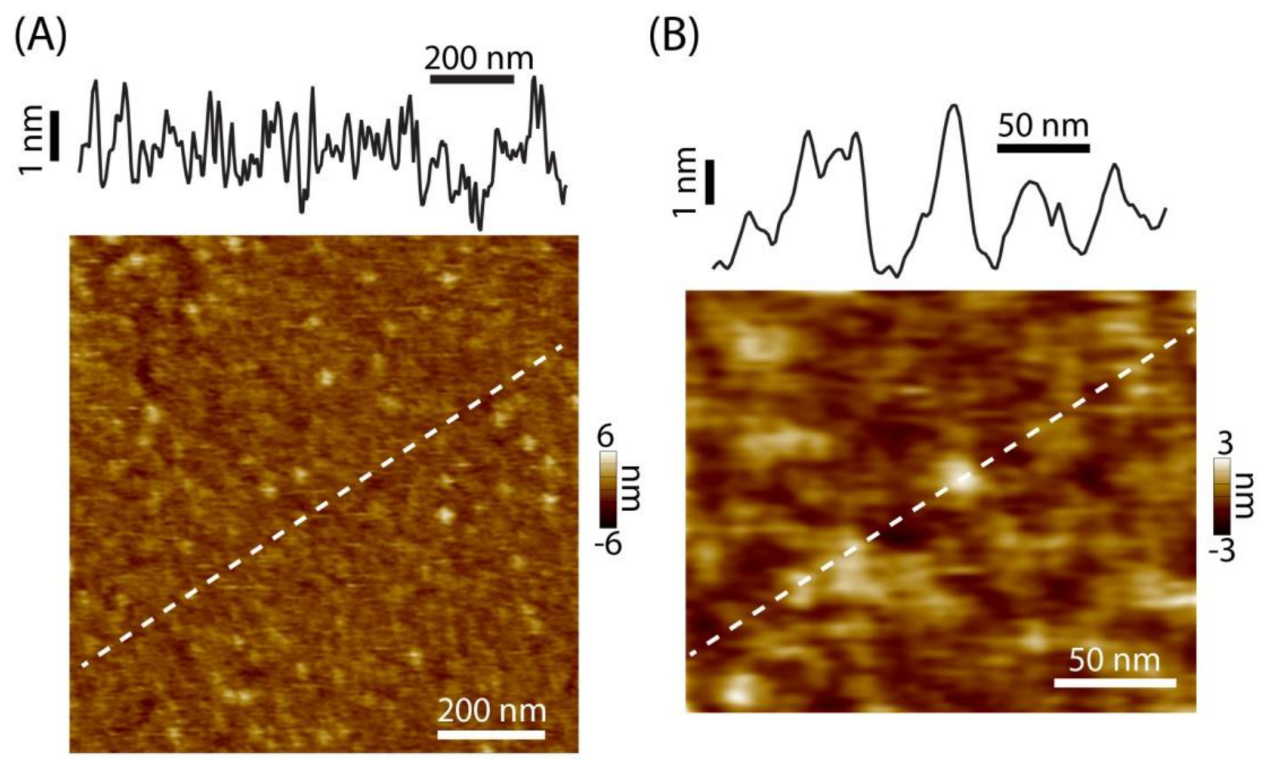

Figure 10: Evaluation of molecular density of SecA prepared for surface adsorbed ATP hydrolysis activity assays. (A) AFM image of SecA prepared for surface based ATP hydrolysis measurements. The image illustrated that the surface was completely covered. (B) showed a detailed view of SecA on mica surface. 


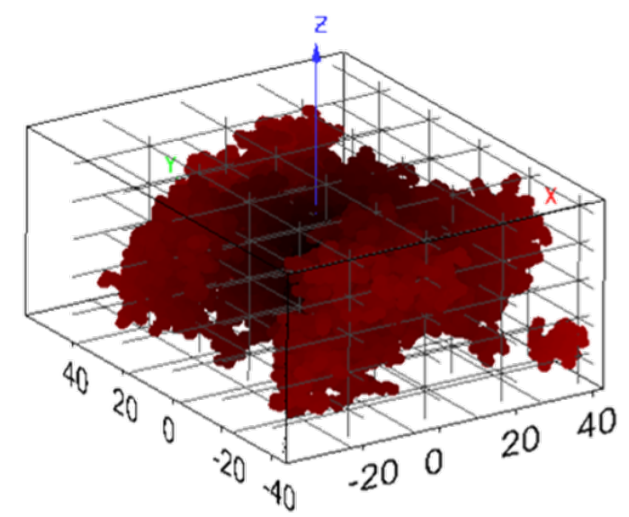

Figure 11: SecA (PDB code 1M6N) was enclosed in the rectangular grid to highlight the dimension of the protein on the surface. The approximate areal footprint dimension was $8 \mathrm{~nm} \times 10 \mathrm{~nm}$. [24]

Figure 10A illustrates that the mica surface was completely covered with SecA using AFM. A magnified view of the same surface is shown in 10B. Figure 11 shows the crystal structure of SecA using crystallographic data obtained from a protein data bank with PBD code 1M6N. It indicates that SecA's areal footprint dimension was $8 \mathrm{~nm} \times 10 \mathrm{~nm}$. The ratio of the surface area and SecA's dimension were used to calculate moles of SecA available on the surface. The estimated number of moles of SecA on the surface was 3 pmol.

\subsection{Translocase Activated ATP hydrolysis}

The next level of ATP hydrolysis is translocase activated ATP hydrolysis. To achieve this level of ATP hydrolysis, SecA must form a complex with SecYEG in E. coli membrane called the translocase. The binding of SecA to the translocon SecYEG is thought to create a conformation that allosterically accelerates the ATP hydrolysis rate [27]. To show this, we employed the use of reconstituted proteoliposomes of SecYEG co-assembled with SecA (Y·A). This reconstitution system showed a high level of activity similar to that of inner membrane vesicles [7,41,46]. Figure 12 shows the 
experimental set up for the translocase activated ATP hydrolysis. Proteoliposomes Y.A were adsorbed on freshly cleaved mica. Experimental conditions were identical to those discussed in the basal ATP hydrolysis. Appendix A.2 lists detailed procedure of the on-surface translocase activated ATP hydrolysis experiments.
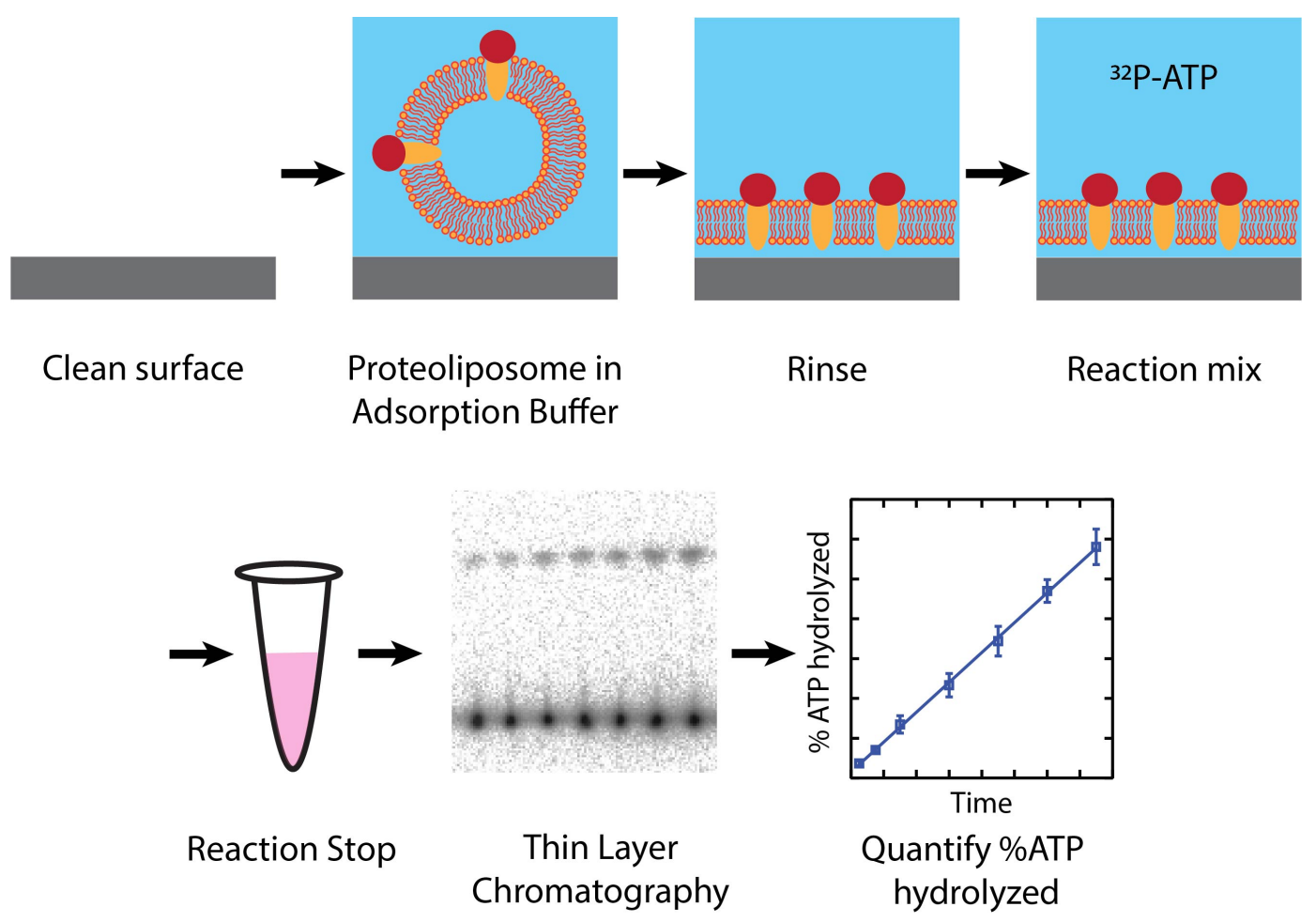

Figure 12: Proteoliposomes Y.A were adsorbed on freshly cleaved mica. The surface was rinsed. The reaction mix containing radioactive ${ }^{32} \mathrm{P}$ was added to the surface. At determined times, the surface solution was sampled and mixed with reaction stop. Thin layer chromatography was performed and the \%ATP hydrolyzed was quantified. Normalization was also carried out for meaningful comparison [68] 


\section{Results}
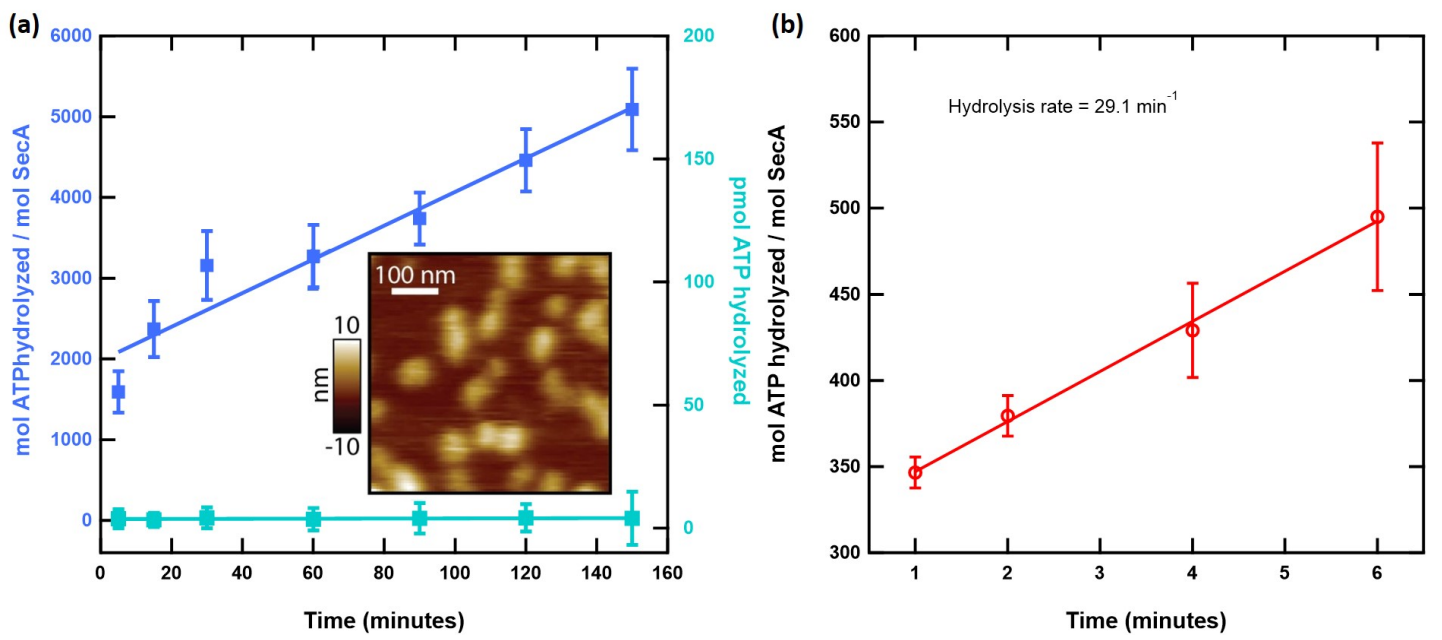

Figure 13: (a) Normalized ATP hydrolysis activity of the translocase complexes adsorbed on mica surface. The inset is the AFM image of the area prepared with identical conditions to the activity measurements. (b) Normalized ATP hydrolysis activity of the system prepared in solution. Slopes of both curves represent rates of hydrolysis activity, which were comparable.

Figure 13 compares surface translocase activated ATP hydrolysis assays to the standard solution assay. Samples in the two assays were prepared under the identical buffer conditions. The activities were normalized in order to make a direct comparison between the two assays. The translocases on the surface hydrolyzed ATP at a significantly higher rate than the control, which lacks the translocases, as shown in the solid and dashed lines in Figure 13a, respectively. The complexes on the surface hydrolyzed ATP at a comparable rate with those in solution shown in 13b [69]. 


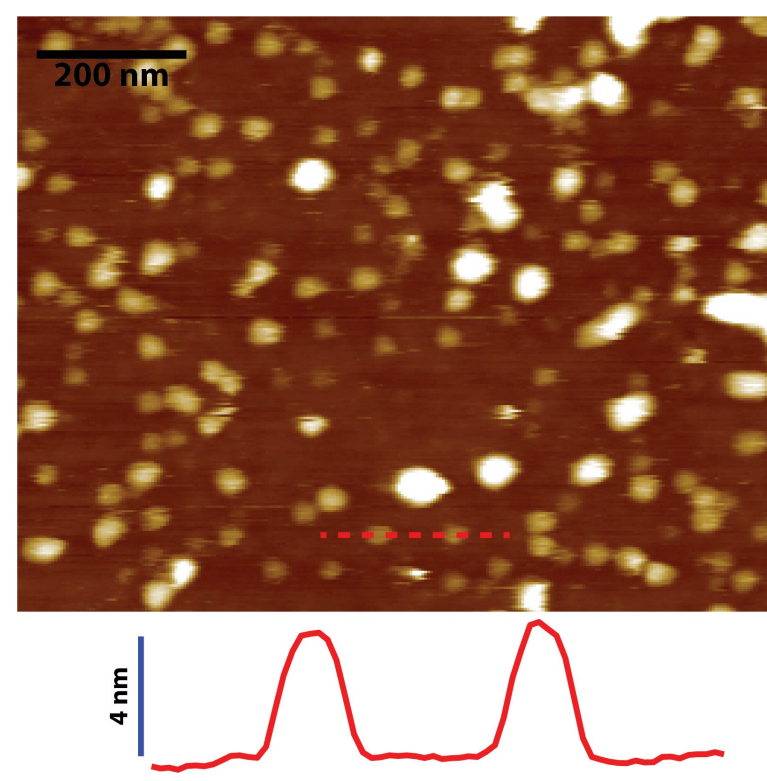

Figure 14: The red dashed line is the line profile shown at the bottom of the representative AFM image of surface-adsorbed translocase complexes.

Figure 14 displays representative AFM image of the translocase complexes on the surface. The image shows more or less complete coverage of the proteoliposomes on the surface. Only those protrusions above the background whose heights fitted within the translocase complex range (greater than or equal to $4 \mathrm{~nm}$ ) were counted toward quantification of available translocase complexes on the surface. We estimated the amount of translocase complexes on the surface to be about 0.03 pmol.

\subsection{Translocation Associated ATP hydrolysis}

The highest level of ATP hydrolysis activity is expected when precursor proteins and SecB are added to proteoliposomes Y.A $[63,70]$. This forms a full system with activity levels comparable to SecYEG in a native environment, inner membrane vesicles, after accounting for the fraction of accessible translocons, the orientations of which are randomized during reconstitution $[7,46,71]$. To demonstrate enhanced activity during translocation, we included precursor proteins and chaperone SecB 
(which minimizes premature precursor folding) to the surface ATP hydrolysis assay (Figure 15). Two precursor species were employed: precursor of outer membrane protein A, pOmpA and precursor of galactose binding protein, pGBP. TLC analysis was carried out as described in the previous sections. Detailed translocation associated ATP hydrolysis experimental procedure is listed in Appendix A.3

Two types of surfaces were used to compare the effect of the surface on the translocation associated ATP hydrolysis activities. In particular, we tested mica surfaces as well as treated glass surfaces. The glass surface treatment is listed in Appendix A.5.

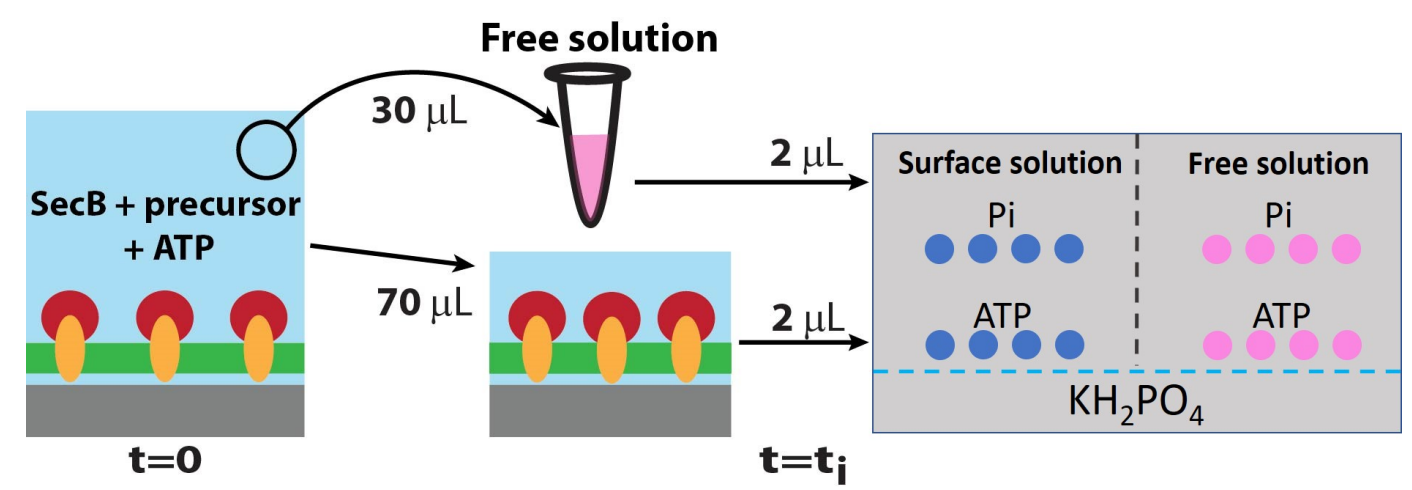

Figure 15: Proteoliposomes Y.A were adsorbed on mica and rinsed. A solution containing SecB, precursor protein (pOmpA or pGBP), and radiolabeled ATP was added. Immediately thereafter, $30 \mu \mathrm{L}$ of solution was transferred to a separate tube (free solution). At subsequent times, solutions were sampled, mixed with EDTA to stop the reaction, and analyzed via TLC. 


\section{Results}
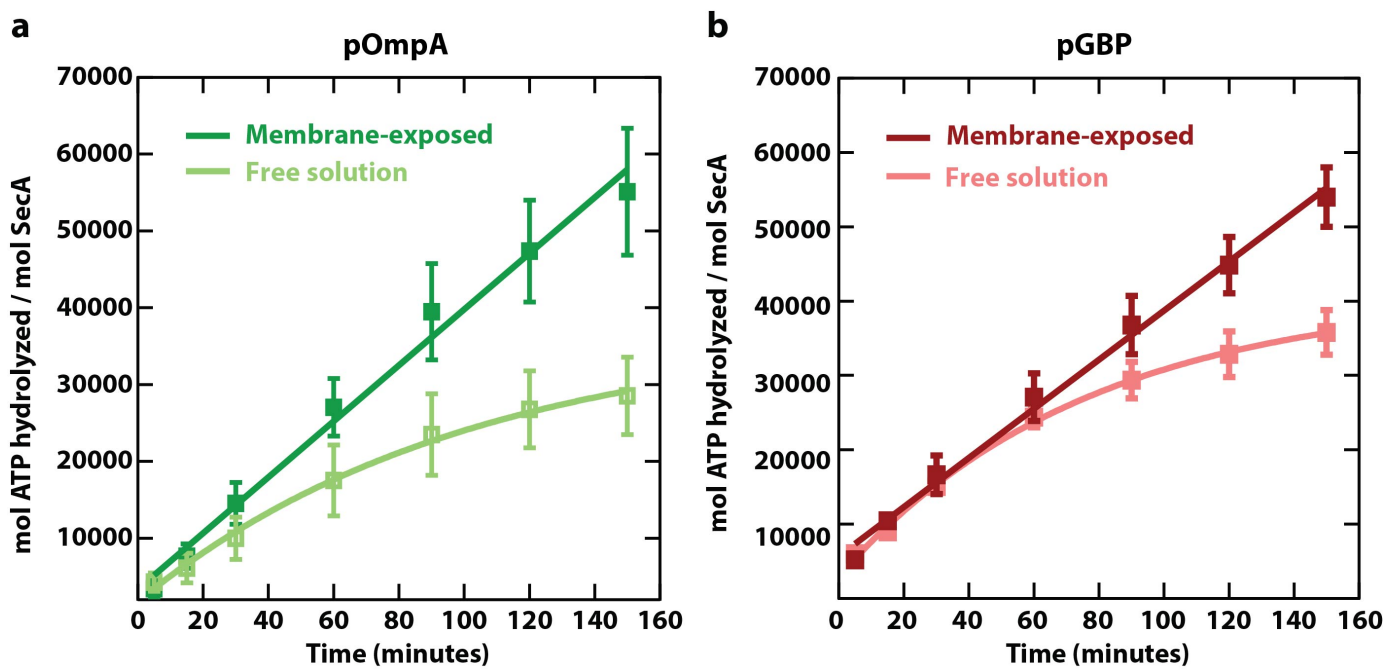

Figure 16: (a) Plot of ATP hydrolyzed as a function of time with pOmpA from the membrane-exposed solution (dark green) and free solution (light green). (b) Analogous plot with pGBP for the membrane-exposed solution (dark red) and free solution (light red). Data normalized to mol SecA available, error bars were SD, N

$=3$. The hydrolysis rates in the membrane-exposed solutions were 364 and 330 $\mathrm{min}^{-1}$ for $\mathrm{pOmpA}$ and $\mathrm{pGBP}$, respectively.

Translocation-associated ATP hydrolysis data for translocation of pOmpA and pGBP in mica-supported lipid bilayers are shown (Figure 16a, b, respectively). The slopes of these curves represent the rates at which inorganic phosphate (Pi) was released from ATP, normalized to the estimated amount of SecA available to participate in the reaction. As expected, the difference between ATPase rates with each precursor was minimal [46]. The translocation-associated ATP hydrolysis rate with pOmpA was $(364 \pm 17) \mathrm{min}^{-1}$ while the rate with pGBP was $(330 \pm 11) \mathrm{min}^{-1}$. For both precursors, the translocation associated rate was more than an order of magnitude higher than the translocase-activated rate and $>40$-fold higher than the basal ATP hydrolysis rate that was previously measured for SecA [24].

As a peripheral ATPase, SecA is known to dissociate from the membrane during translocation $[26,41,72]$ and this population of SecA should continue to hydrolyze 
ATP, but at what level? To evaluate this, immediately after adding radiolabeled ATP, a $30 \mu \mathrm{L}$ volume of solution above the mica surface (Figure 15, free solution) was moved to a microcentrifuge tube for quantification in parallel with the membraneexposed volume. Surprisingly, the free solution data for both precursor species exhibited highly activated hydrolysis levels for extended time periods [Figure 16a, b, light green (pOmpA) and light red (pGBP)]. This population of SecA had been exposed to the full Sec system for $\sim 1$ min before being separated from the membrane-bound SecYEG; yet, the hydrolysis rates essentially matched the translocation-associated rates for tens of minutes before decreasing. It is noted that surface-adsorbed samples were rinsed extensively prior to beginning the assay; thus, it was not likely that SecYEG detached from the surface during the assay. Further, exposure of SecA to precursor protein, which was likely in the free solution in the absence of SecYEG, did not activate SecA ATP hydrolysis [71].

Table 1: Table comparing ATP hydrolysis activities on mica vs in solution, data from refs $[24]^{*},[69]^{* *},[70,71]^{* * *}$, and $[46,63,71]^{* * * *}$.

\begin{tabular}{|c|c|c|}
\hline & $\begin{array}{l}\text { Surface ATP hydrolysis rate } \\
\text { mol ATP hydrolyzed } / \mathrm{molSecA} / \mathrm{min}\end{array}$ & $\begin{array}{l}\text { Solution ATP hydrolysis rate } \\
\text { mol ATP hydrolyzed } / \mathrm{molSecA} / \mathrm{min}\end{array}$ \\
\hline Basal & $7.8^{*}$ & $8.3^{*}$ \\
\hline Translocase & $21^{* *}$ & $\sim 24^{* * *}$ \\
\hline Translocation & $330-360^{* *}$ & $\sim 460^{* * * *}$ \\
\hline
\end{tabular}

It is informative to compare ATPase rates to those measured in traditional solution based assays $[46,63,70,71]$. As shown in Table 1 , the hydrolysis rates under all levels of activation were comparable to those observed in traditional assays. In particular, the translocase-activated and translocation-associated rates were $>70 \%$ of the solution rates. The overall agreement between these assays implies that the majority of accessible YEG·A complexes remained competent for ATP hydrolysis and released 
Pi at a rate similar to that achieved in solution. Additionally, despite the small number of translocase units participating, the surface-based assay had sufficient sensitivity to distinguish different levels of ATP hydrolysis. Samples supported by glass coverslips showed similar hydrolysis activities to mica supported samples in both the free solution as well as in the membrane-exposed volumes shown in Figures 17a and b. The choice of surfaces species did not appear to affect ATP hydrolysis activities significantly. The percent ATP hydrolyzed on mica and glass surfaces are shown in Figure 17a. The normalized ATP hydrolysis activities are shown in Figure 17b. The free solution samples were also quantified on glass with $\mathrm{pOmpA}$ precursor protein in an identical manner as those done on mica (Figure 18). The data recapitulate the same hysteretic behavior as the system on mica.

a

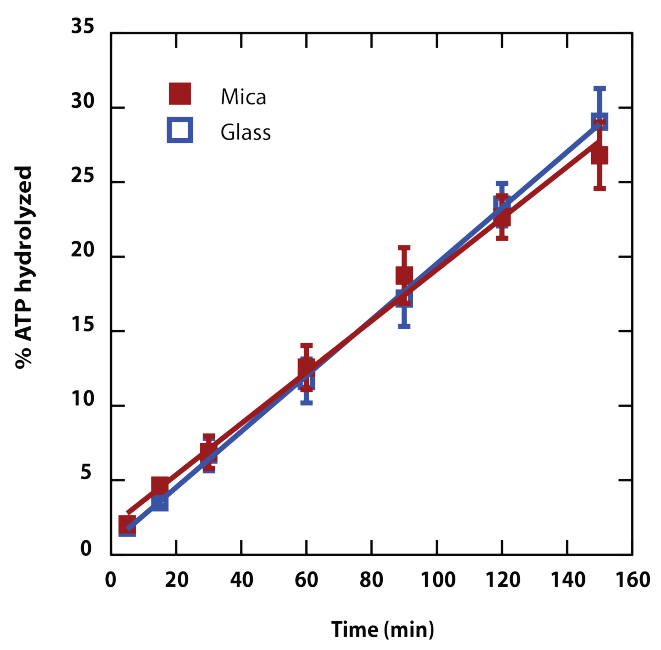

b

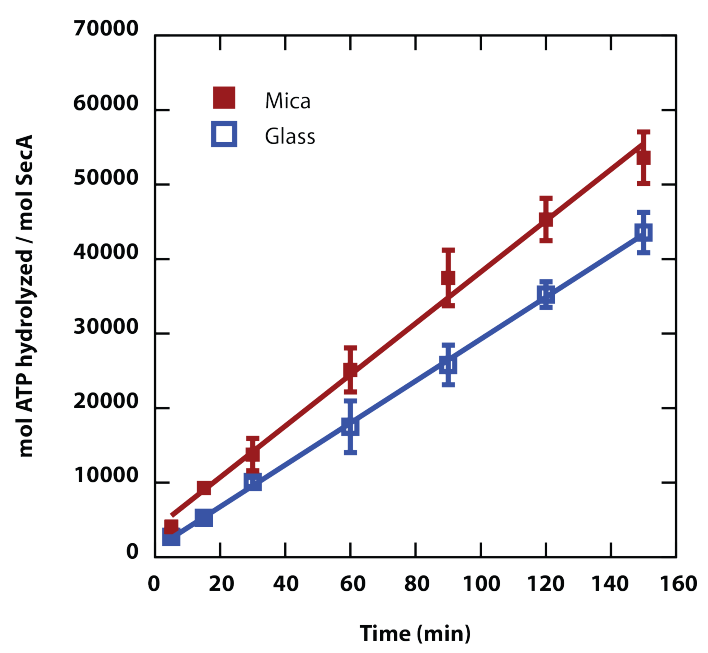

Figure 17: Proteoliposomes Y·A were deposited on either freshly cleaned borosilicate glass or freshly cleaved mica. ATP hydrolysis activity was assessed in the presence of pGBP and SecB 


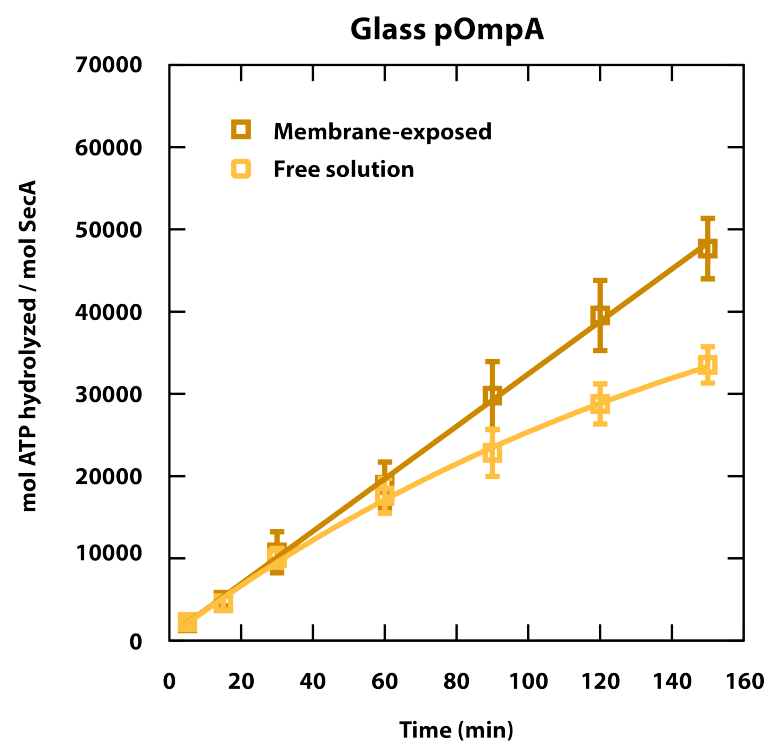

Figure 18: Proteoliposomes Y·A were deposited on either freshly cleaned borosilicate glass. ATP hydrolysis activity was assessed in the presence of pOmpA and SecB for membrane-exposed volumes (dark brown squares) and free solution (light brown squares). Error bars are $\mathrm{SD}, \mathrm{N}=3$.

This SecA activation was independent of the substrate surface species. As demonstrated here, surface-based activity assays allow straightforward separation between membrane-exposed fractions and free solution fractions. This can be viewed as a general advantage of such assays.

In order to properly discuss our on-surface ATP hydrolysis activity assays, we compared the activity levels with the traditional in-solution assay. The in-solution ATP hydrolysis assays were done in a systematically identical manner to our onsurface ATP hydrolysis assays: basal, translocase activated, and translocation associated ATP hydrolysis activities. Detailed experimental protocols for in-solution ATP hydrolysis assays are provided in Appendix B.1. 


\section{Translocation activities}

The coupling between chemical energy consumed (ATP hydrolysis) and work done moving polypeptides across the membrane is not tight in the Sec system and has been shown to vary with precursor species [46]. Hence, to complement ATP hydrolysis measurements, we developed a surface-based translocation activity assay that tracked the protection of precursor proteins in an ATP-dependent manner [41, 73]. Translocation is demonstrated on two distinct supporting surfaces. We compare results using mica, which is atomically flat over large areas, to cleaned borosilicate glass coverslips, which exhibit topographic asperities that may provide additional submembrane space for the precursor to occupy [8].

\subsection{Surface translocation activities assay}

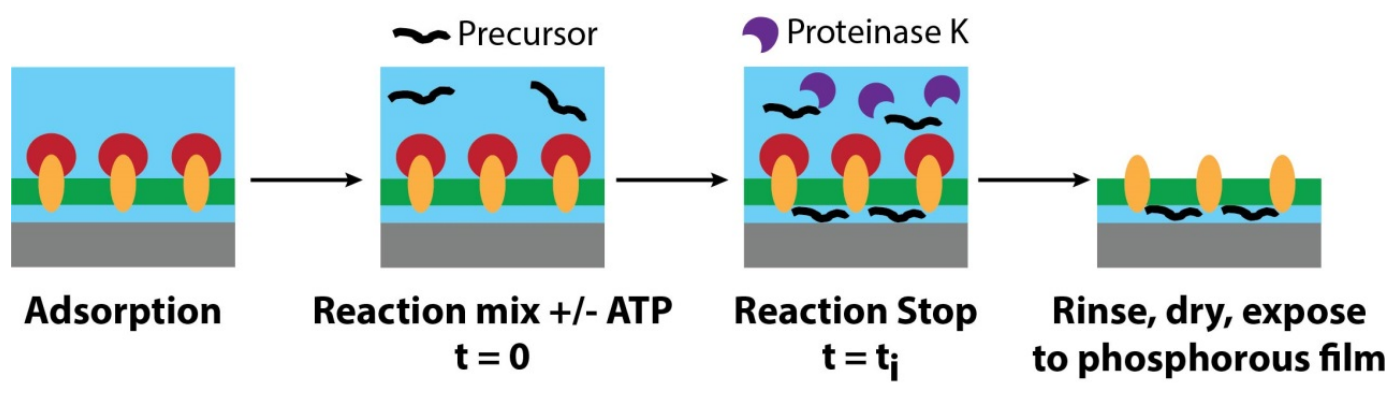

Figure 19: Schematic of surface translocation activity assay used to quantify amount of protected radiolabeled precursor protein (black) in the presence and absence of ATP $( \pm \mathrm{ATP})$.

Because proteinase $\mathrm{K}$ does not cross the lipid bilayer, translocation can be inferred via protection of radiolabeled precursors from proteinase $\mathrm{K}$ addition above the membrane surface (Figure 19 [73]). A brief overview of the assay follows. Proteoliposomes Y·A were adsorbed onto either freshly cleaned glass coverslips or freshly cleaved mica surfaces, creating supported lipid bilayers over large areas [8]. After 
rinsing to remove the loosely adsorbed material, a reaction mixture containing SecA, $\mathrm{SecB}$, and radiolabeled precursor (pOmpA or pGBP) was added in the presence and absence of ATP. At predetermined times, surfaces were treated with urea (a denaturing agent) to destabilize the proteins and render them sensitive to proteolysis by added proteinase K. Finally, surfaces were rinsed, dried, and exposed to a phosphor imaging plate for analysis. Step by step details of the experimental procedure are provided in Appendix A.4

\section{Results}

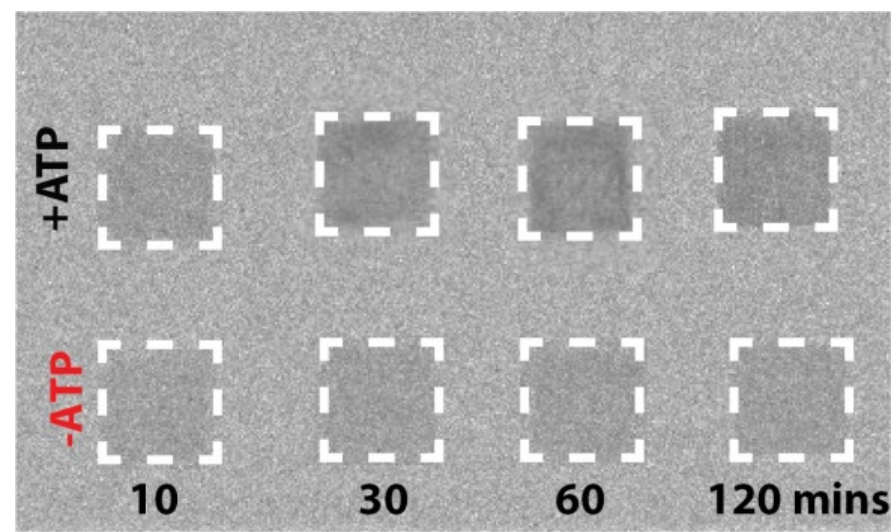

Figure 20: Phosphor image of glass surfaces at indicated times. The location of the glass is highlighted (dashed squares). The systems were compared in the presence $(+)$ and absence (-) of ATP 


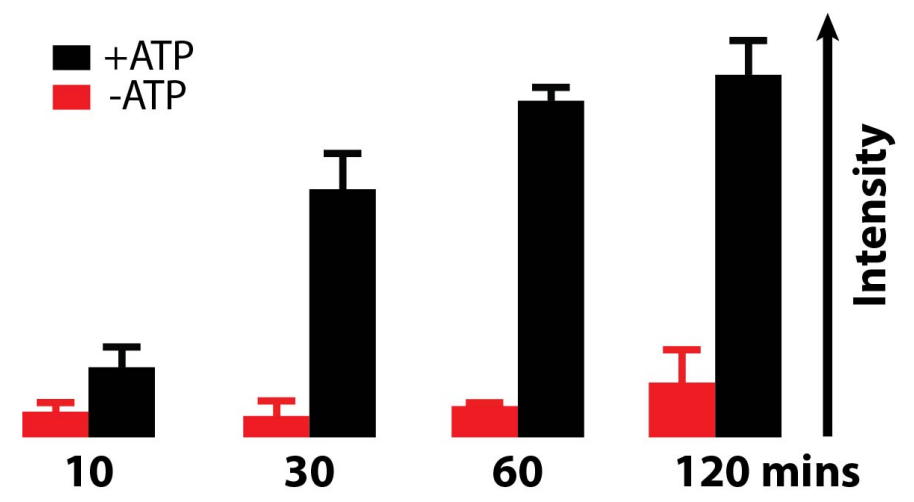

Figure 21: Bar graphs indicate intensities of protected pOmpA in the presence and absence of ATP as a function of time on glass surfaces. The observed increase in intensity as a function of time is a hallmark of active translocation and indicates that the system remains active when adsorbed to a supporting surface.

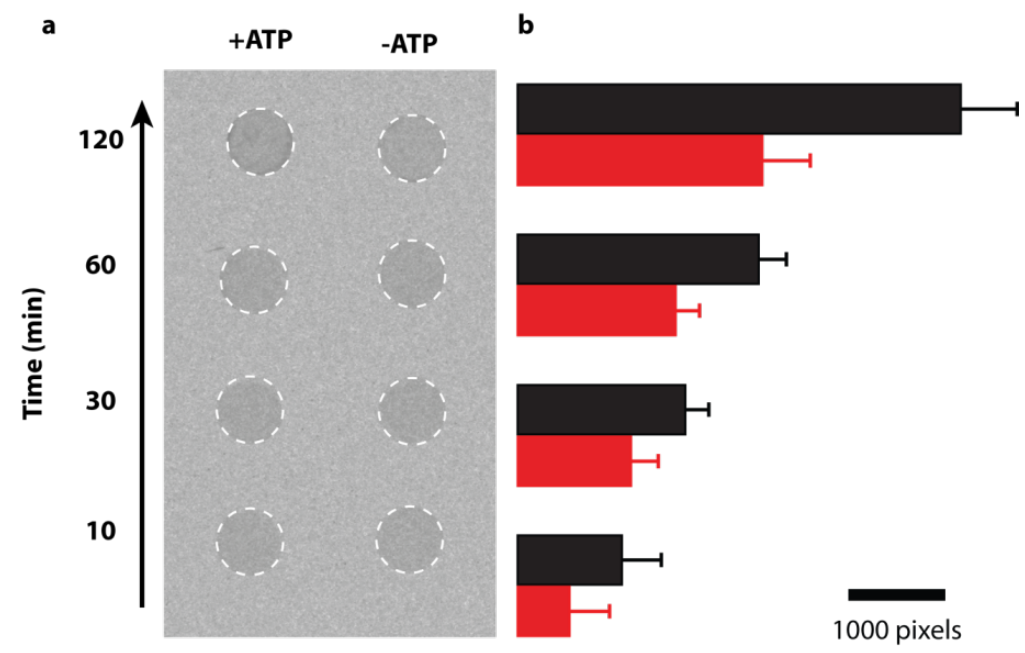

Figure 22: Translocation activity assays were performed on mica in an identical manner to those on glass. (a) Phosphor image data for pOmpA on mica. Dashed circles highlight the area of each mica disk. (b) Bar graphs represent the integrated intensity of pixels inside the dashed circles at corresponding time points (black $=$ + ATP, red $=-$ ATP). 

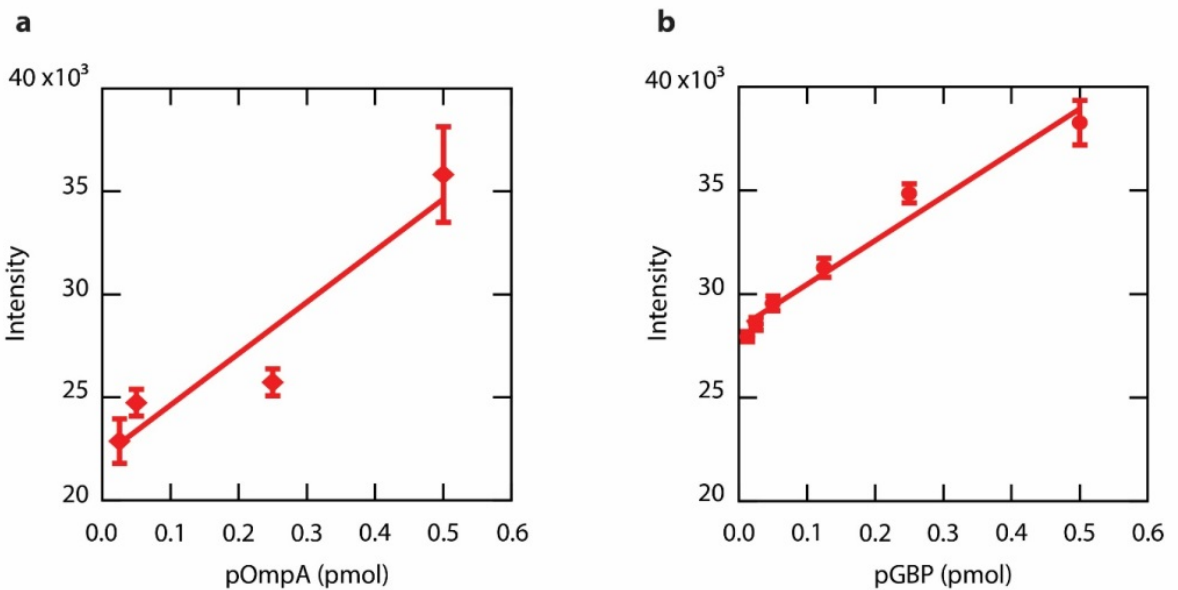

Figure 23: Calibration curves were used to convert intensity of the phosphor image to pmol precursor protected. (a) shows a standard curve for pOmpA, (b) for pGBP. Intensity was fitted with a linear function of concentration. Surfaces prepared for standard curves were placed in the same cassette as those obtained from the translocation activity experiments. Therefore, all surfaces were subjected to the same exposure time and environment (identical background).
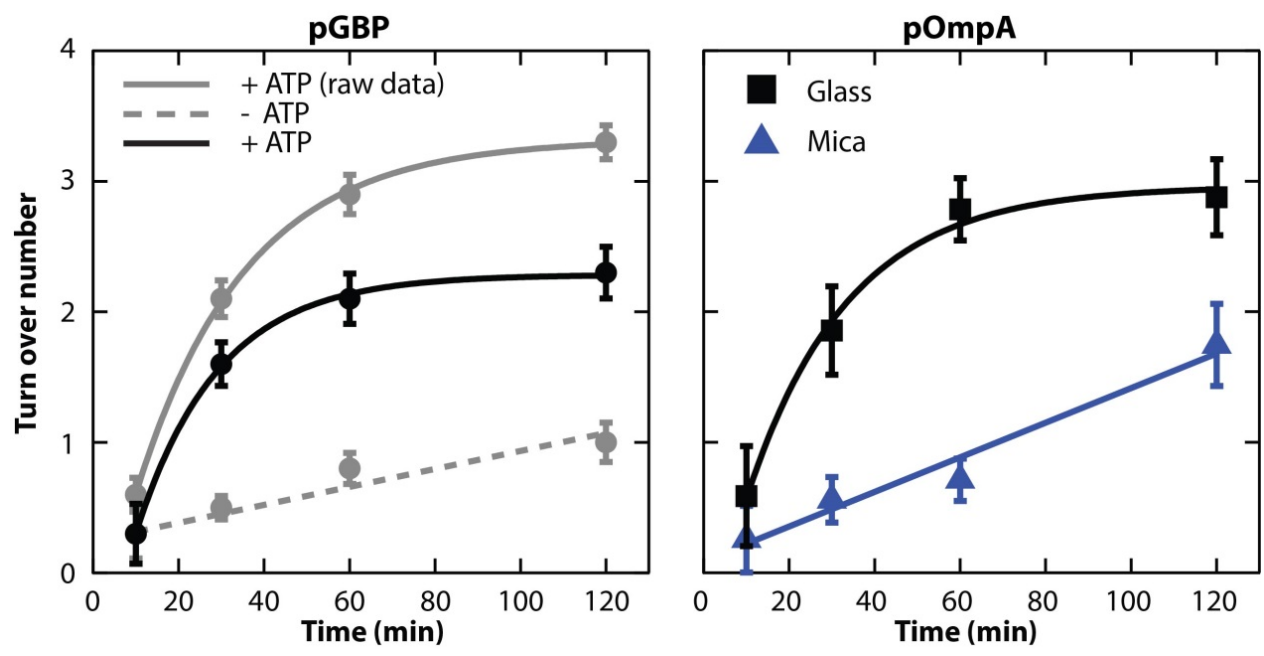

Figure 24: Turn over number (mol precursor protected per mol translocase accessible) as a function of time for pGBP on glass coverslips. Raw data (gray solid line) as well as data from which the - ATP background was subtracted (black solid

line) are shown (left). Background subtracted plots for pOmpA on glass (black squares) or mica (blue triangles) (right). Error bars are $\mathrm{SD}(\mathrm{N}=3)$.

The amount of radioactivity exhibited by the protease treated membrane was quantified. Figure 20 shows data obtained from the system with pOmpA on borosil- 
icate glass. The dashed squares highlight areas where the coverslips were placed on the imaging plate. The integrated intensities are shown in bar graphs in Figure 21. The data (Figure 20 and 21) show ATP-dependent precursor protection. This is the hallmark of active translocation. Analogous data for pOmpA on mica are shown in Figure 22. We created standard curves of pixel intensity as a function of mol radioactive precursor by spotting known quantities of precursor on surfaces (Figure 23. The calibration surfaces were dried and exposed to the imaging plate under conditions identical to those used to quantify translocation activity. The turn over number versus time is plotted for pGBP and pOmpA on glass (Figure 24). The vertical axis represents mol precursor protected per mol translocase accessible to precursors. Amount of accessible translocase was estimated from AFM images. The amount of translocase accessible was estimated to be $0.04 \mathrm{pmol}$ which is slightly larger than the value for mica due to surface area differences. For both precursors, the translocation activity as a function of time exhibited similar exponential rise to maximum behavior, reaching a plateau between 2 and 3 translocation cycles with apparent rate constants of $(0.052 \pm 0.004)$ and $(0.042 \pm 0.009) \mathrm{min}^{-1}$ for pGBP and pOmpA, respectively. Values were calculated after subtracting the -ATP background, and errors are $68 \%$ CI estimated from non-linear least squares fits, $\mathrm{N}=3$. Note that $\mathrm{CI}$ is confidence interval. The $68 \%$ represents the errors obtained from one standard deviation of the measurement. All error bars shown in every figure are standard deviation. Apparent rate constants represent the rate constant of the slowest step in the cycle; more generally, they express the probability of a translocation event per unit time.

The translocation activity results varied significantly with the supporting surface (Figure 24). With mica, the extent of translocation did not reach a plateau but was linear over the time tested. At 120 min, it was only 60\% of the glass result. The slope of the pOmpA turn over number versus time plot on mica was $(0.013 \pm 0.002)$ 
$\min ^{-1}, \mathrm{~N}=3$, representing the mol pOmpA protected per minute normalized to the estimated mol translocase accessible on mica. This slope was fivefold lower than the initial slope on glass, which stayed approximately linear for 30 min before beginning to plateau (Figure 24, compare black squares to dark blue triangles).

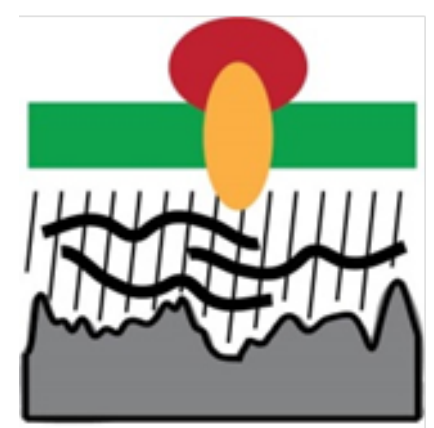

Glass

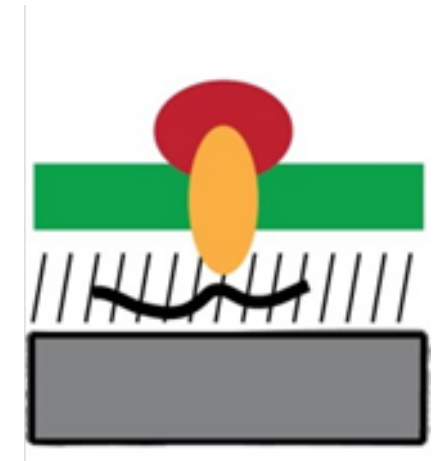

Mica

Figure 25: Cartoons (not to scale) showing how the rougher underling topography of glass could create space that enhances translocation activity in comparison to mica.

These surface dependent results may be attributable to distinctions in underling topography. The cleaned borosilicate glass coverslips we utilized are approximately fivefold rougher than mica [8]. Additional roughness likely led to valleys in the submembrane space that precursors could move into, promoting more turn over events per unit time (Figure 25). Additionally, though both surfaces are highly hydrophilic, differences in surface chemistry and charge could also play a role. We posit that the lower effective rate constant observed on mica compared to glass is likely a result of a high level of confinement between the mica and the surface-proximal lower bilayer leaflet. Increased confinement produces a larger effective drag force on the translocating precursor, resisting motion. 


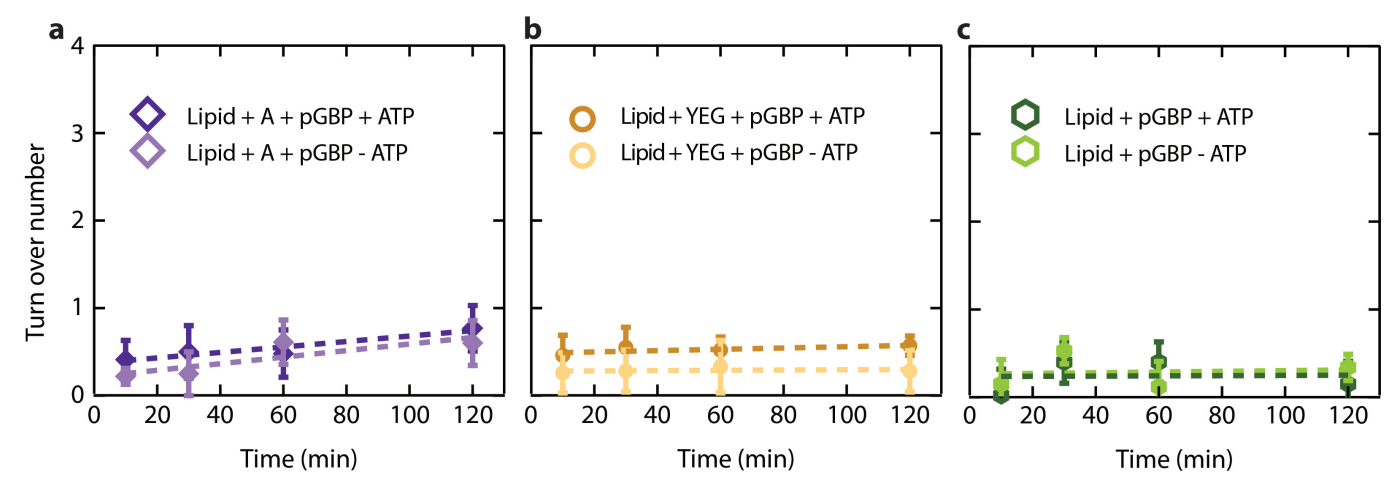

Figure 26: Both SecYEG and SecA were required for translocation activity. Assays carried out on glass surfaces with all of the required components of the general Sec system excepting SecYEG (a), SecA (b), or (c) both SecYEG and SecA. All experiments showed minimal translocation as evidenced by the lack of ATP-dependent protection of precursor pGBP. Best fit lines (dashed) were shown, error bars were SD $(\mathrm{N}=3)$.

Experiments were conducted to verify that the results observed reflect translocation activity through SecYEG. Both the translocon SecYEG and ATPase SecA were required to achieve active translocation in surface-supported assays. To show this, we probed the translocation activity of the full Sec system on glass, omitting either SecYEG (Figure 26a), SecA (Figure 26b), or both SecYEG and SecA (Figure 26c). As expected, in all three cases, no significant ATP-dependent protection of precursors was observed. As an additional control, we used AFM to image defects (noncomplete coverage) in the supported lipid bilayers, as changes in defect number or defect area with the addition of ATP could give rise to erroneous deductions of translocation. To generate defects for this experiment, surfaces were incubated for short periods (20 min). The data show no gross differences in bilayer defects \pm ATP (Figure 27); however, technical challenges associated with finding the same region of the surface before and after ATP addition make this experiment challenging to perform. 

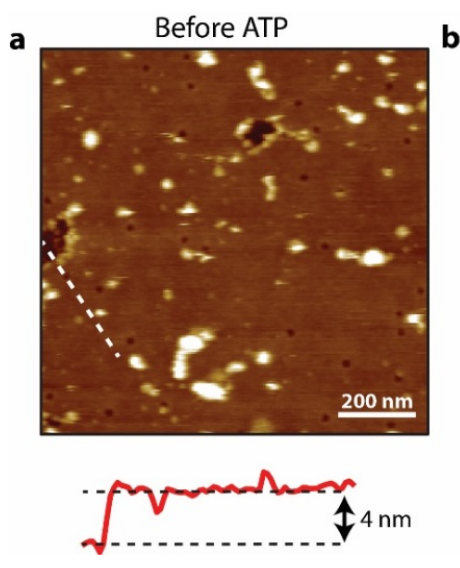
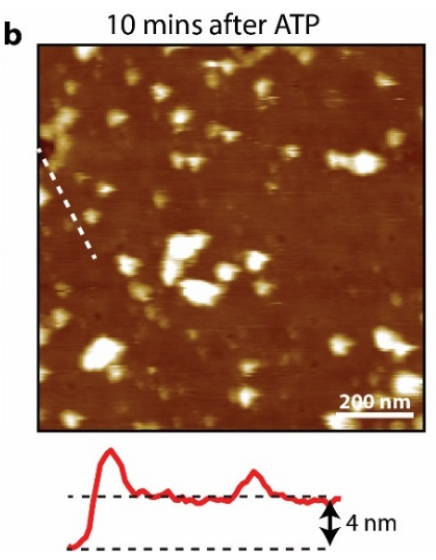
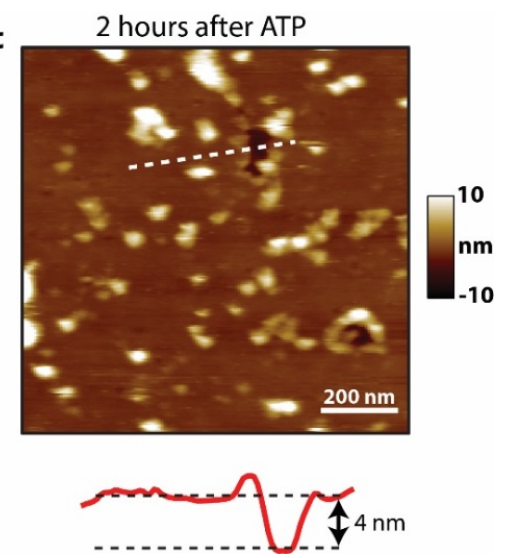

Figure 27: Representative AFM images of proteoliposomes Y·A on glass surfaces (a) before, (b )10 minutes after, and (c) two hours after adding $3 \mathrm{mM}$ ATP into the imaging buffer. The same location of the lipid bilayer surface (with an offset) was shown in (a) and (b). (c) showed a different area of the bilayer. The line scans (red, location in image: white dashed) below each image indicate the height of the lipid bilayer $(4 \mathrm{~nm})$ remained unchanged. Overall, the data suggested that the addition of ATP did not grossly alter the bilayer defects. [41]

Comparisons of the extent of translocation (the maximal amplitude of the reaction) measured on glass coverslips to solution-based translocation assays using the same Sec system components were in close agreement (within a factor of two, determined by fitting the data to a single exponential rise to maximum [46]). However, the apparent rate constants were reduced approximately 10- fold [46]. This rate constant reduction is likely due to an effective frictional drag force on the translocating precursor from the nearby solid-state surface. Data with borosilicate glass were fit to a single exponential rise to maximum with the equation $y(t)=y_{0}+A\left(1-e^{-k\left(t-t_{0}\right)}\right)$, where A was the maximal amplitude of the reaction, $k$ is the apparent rate constant, and $t_{0}$ corrects for the initial time lag $(\sim 15 \mathrm{~s})$. Data with mica were fit to a line. All experiments were performed at least three times, and standard deviations are shown as error bars. The on-surface translocation activity was compared with the traditional translocation activity. Figure 28 shows an example of the in-solution translocation activity curve. Appendix B.2 provides a detailed in-solution translocation experimental 
procedure.

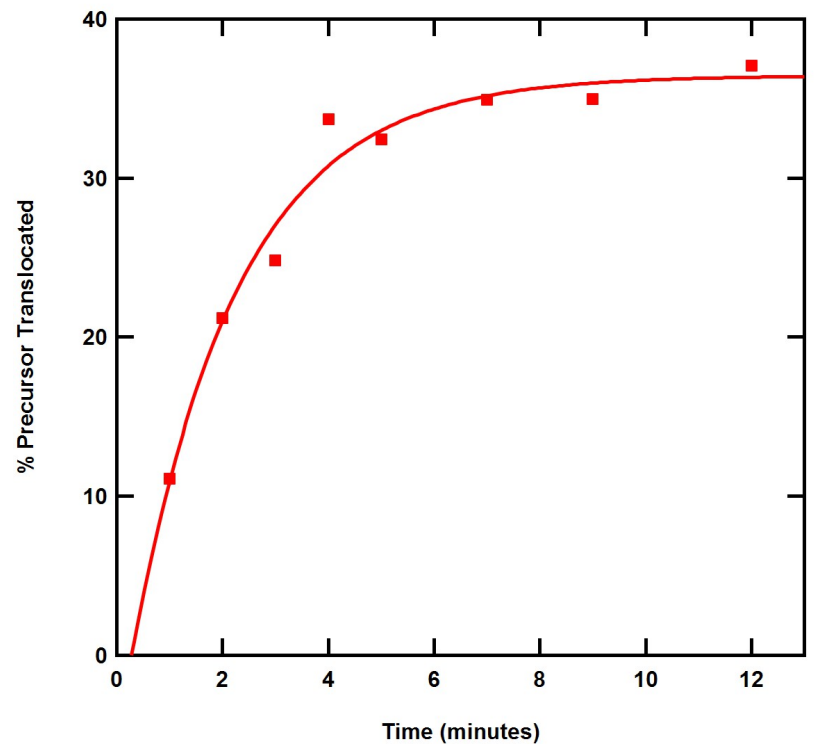

Figure 28: Data showing the translocation activity as a function of time for pOmpA in solution. The graph was fitted with exponential rise to maximum $y(t)=y_{0}+A\left(1-e^{-k\left(t-t_{0}\right)}\right)$. $\mathrm{k}$ represented apparent rate constant of the translocation activity. $\mathrm{k}$ was determined to be $0.5 \mathrm{~min}^{-1}$.

To summarize, glass-supported translocases maintained translocation activity, but with a slower rate-limiting step than solution-based assays. The same statement is true for translocase on mica surfaces, except that the rate-limiting step was even slower, presumably due to tighter submembrane confinement. It is not clear if the rate-limiting step observed on a surface is the same as that observed in solution. It is possible that a different step could become rate-limiting in surface coupled assays compared to assays carried out in solution. The translocation activity persisted on both mica and glass surfaces for extended time periods $(\sim 1 \mathrm{~h})$.

\subsection{Chemo-mechanical coupling}

The energy provided by the hydrolysis of ATP is required for translocation of precursor proteins across the bilayer. Following the convention in the field that pre- 
cursor protection comes about from transportation across the lipid bilayer [73], the translocation-associated ATP hydrolysis and translocation activity assays were used to deduce chemomechanical coupling. On glass surfaces, we obtained chemomechanical coupling of approximately 13 and 15 ATP molecules hydrolyzed per amino acyl residue translocated for pGBP (332 residues) and pOmpA (346 residues), respectively. In solution assays with $\mathrm{Y} \cdot \mathrm{A}$ as well as with inner membrane vesicles, which are taken to represent a native environment for SecYEG, these values are approximately 6 and 8 for pGBP and pOmpA, respectively [46]. Hence, the effect of adsorption onto glass caused an approximate 50\% change in chemomechanical coupling compared to solution assays. However, when proteoliposomes Y.A was adsorbed onto mica, a chemomechanical coupling value of approximately 80 was obtained for translocation of pOmpA. Therefore, fivefold more ATP was consumed per amino acyl residue translocated on mica compared to glass which might be attributed to tighter submembrane confinement on mica. In the standard $\mathrm{Y}+\mathrm{A}$ reconstitution system used by many research groups, proteoliposomes are constructed from purified SecYEG, and then, SecA is added extraneously. $\mathrm{Y}+\mathrm{A}$ samples assayed in solution hydrolyze more ATP per residue translocated than do coassembled proteoliposomes Y.A adsorbed to glass surfaces. In particular, the values for $\mathrm{Y}+\mathrm{A}$ in solution were 22 and 35 (ATP molecules hydrolyzed per amino acyl residue translocated) for pGBP and pOmpA, respectively [46].

Table 2: Chemo-mechanical coupling of translocation activity comparing between on surface (mica and glass) and in solution assay (traditional assemble and coassemble reconstitution).

\begin{tabular}{|c|c|c|c|}
\hline \multicolumn{2}{|c|}{ mol ATP hydrolyzed per mol amino acid translocated } \\
\hline \multicolumn{2}{|c|}{ On surface } & \multicolumn{2}{c|}{ In solution } \\
\hline mica & glass & Y·A & Y+A \\
\hline 80 & 14 & 7 & 29 \\
\hline
\end{tabular}


Sensitive activity assays quantified the biochemical integrity of model membrane protein complexes in surface-supported lipid bilayers. Both ATP hydrolysis assays as well as translocation activity assays confirmed the activity of the reconstituted Sec system in lipid bilayers supported by either mica or glass. Furthermore, surface-adsorbed translocases remained active for extended time periods $(\sim 1 \mathrm{~h})$. This amount of time is commensurate with the typical data acquisition period of AFM experiments as well as with other precision single-molecule techniques such as optical trapping microscopy.

ATP hydrolysis assays have revealed distinct levels of activity corresponding to previously defined levels in solution assays: basal (low), translocase-activated (medium), and translocation-associated (high). All of these levels were found to be similar to traditional solution assays (within $>70 \%$ ). Interestingly, experiments identified a population of SecA that maintained a high level of ATP hydrolysis for tens of minutes despite being released from the membrane surface. This observation is suggestive of activation of SecA by a conformational change that slowly converts back to an unstimulated basal state.

Translocation assays on glass revealed the same exponential rise to maximum behavior observed in solution assays. Quantitatively, the extent of translocation on glass was similar to solution; however, the kinetics was slower. An order of magnitude reduction in the apparent rate constant of translocation was observed on glass compared to solution. This indicates that when the apparatus functions in close proximity to glass, a change occurs in either the rate-limiting step or the features of the step. Future work will be required to deconvolve these possibilities.

The chemomechanical coupling between energy consumed (ATP hydrolyzed) and work done (precursor translocated) only differed twofold on glass when compared to solution. By this metric, coassembled proteoliposomes $\mathrm{Y} \cdot \mathrm{A}$ in glass supported lipid 
bilayers performed more efficiently (consumed less ATP per residue translocated) than traditional solution assays with the standard reconstituted $\mathrm{Y}+\mathrm{A}$ samples that have long been used in the field. The percentage of active translocons is significantly lower in $\mathrm{Y}+\mathrm{A}$ samples $(10 \%)$ compared to $\mathrm{Y} \cdot \mathrm{A}(55 \%)$, and SecA can continue to hydrolyze ATP nonproductively when engaged with translocation defective SecYEG [46]. Hence, one may expect that the chemomechanical coupling of the $\mathrm{Y}+\mathrm{A}$ system would be loose.

Glass was shown to be a more suitable surface than mica for translocation. On average, each accessible translocon on glass underwent just under 3 cycles of pOmpA translocation while those on mica underwent $<2$ cycles. This difference is likely due to the inherent surface roughness of glass. Topographic asperities may lower the effective drag force experienced by the translocating precursor by providing more submembrane space for the polypeptide to occupy. In summary, precision surfacecoupled assays are poised to uncover mechanisms of biochemically active Sec translocases. More generally, translocation activity assays yield valuable quantification of surface-supported lipid bilayer environments. 


\section{Submembrane characterization via Neutron Re- flectometry}

To complement single molecule atomic force microscopy investigations which provide structural information on surface-distal regions of molecules, we performed neutron reflectometry (NR). In particular, NR provided insights into the submembrane structure of proteoliposomes of SecYEG·SecA adsorbed to $\mathrm{SiO}_{x}$, a material closely related to glass coverslips. Because of instrumentation limitations, we could not utilize borosilicate glass coverslips nor mica in NR measurements. Here, proteoli-

posomes were incubated on a nominally $100 \AA$ thick film of dry thermal silicon oxide $\left(\mathrm{SiO}_{x}\right)$ grown on a single crystal silicon wafer. $\mathrm{SiO}_{x}$ was chosen as a closely related surface proxy for borosilicate glass. However, these surfaces exhibited topographical and surface chemical differences that might alter behavior, for example, during proteoliposome rupturing and spreading (Figure 29). All NR work reported here was performed at the Center for Neutron Research at the National Institute of Standards and Technology (NIST). We are grateful to Dr. David Hoogerheide at NIST for his significant contributions to this work. 


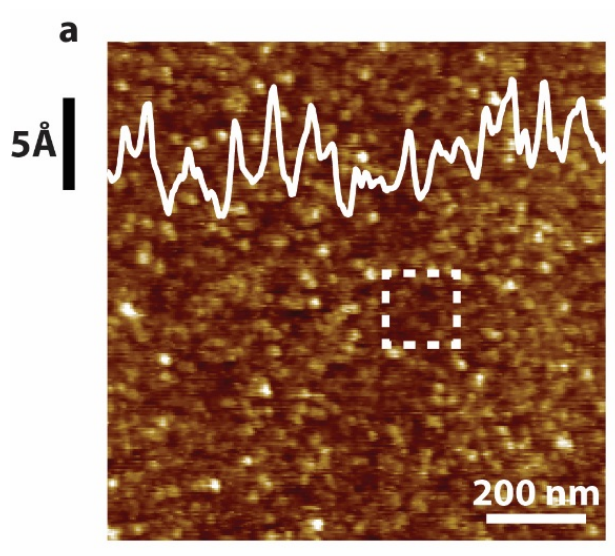

$\mathrm{SiO}_{\mathrm{x}}$

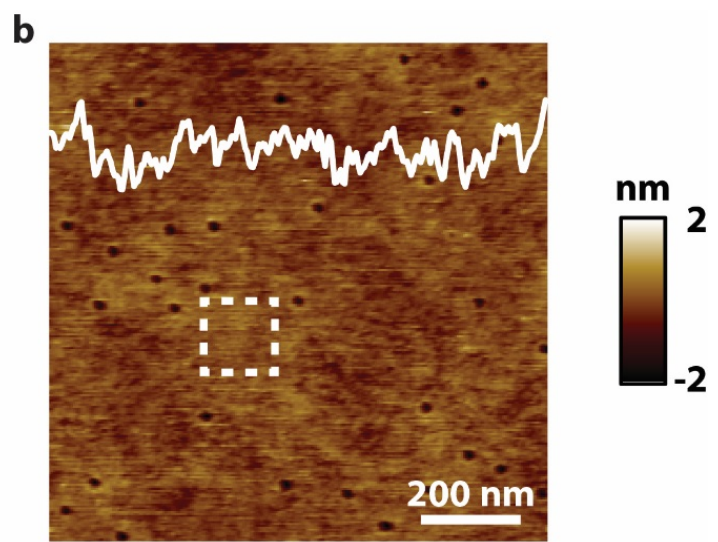

Borosilicate glass

Figure 29: Representative AFM images of $\mathrm{SiO}_{x}$ and borosilicate glass coverslips are shown in (a) and (b), respectively. Line scans (white, with $5 \AA$ vertical scale bar) are also included. Prior to imaging, both surfaces were sonicated in $\mathrm{KOH}$ saturated ethanol for 2 minutes. The borosilicate glass was further treated in oxygen plasma for 10 minutes [8]. Immediately after cleaning, imaging buffer was added and AFM images were taken. Areas of $150 \times 150 \mathrm{~nm}^{2}$ were used to characterized the roughness of the surfaces identified by dashed white squares in (a) and (b). The RMS roughness values were $2.5 \AA$ and $1.7 \AA$ for the $\mathrm{SiO}_{x}$ and borosilicate glass, respectively. The same tip was used to acquire all data. The $\mathrm{SiO}_{x}$ surface was imaged after the borosilicate glass surface. The same tip was then used to image borosilicate glass again. The resulting RMS values were similar, indicating that the overall dimensions of the tip (which were convolved into the RMS analysis) were static during the analysis.

\section{Results}

The $\mathrm{SiO}_{x}$ sample was prepared by chemical vapor deposition (see Appendix $\mathrm{C}$ for details). The surface was treated with saturated ethanolic solution of potassium hydroxide similar to the borosilicate glass surface. Proteoliposomes $\mathrm{Y} \cdot \mathrm{A}$ were adsorbed on the freshly cleaned wafer. The sample wafer was mounted on a flow cell to allow subsequent buffer exchange. NR reflectograms were collected in both $\mathrm{D}_{2} \mathrm{O}$ and $\mathrm{H}_{2} \mathrm{O}$-based buffers which mimicked the translocation activity buffers. Data from four conditions were used for analysis (Figure 30a). NR data of the surfaceadsorbed SecYEG-containing bilayer was analyzed using composition space model- 
ing [74]. The bilayer was assumed to consist of a single dioleoylphosphatidylcholinelike lipid species with an acyl chain volume of $1024 \AA^{3}$, a headgroup volume of $330 \AA^{3}$, an acyl chain scattering length of $-2.67 \times 10^{-4} \AA$, and a headgroup scattering length of $6.00 \times 10^{-4} \AA$. The bilayer was further assumed to contain SecYEG at the preparative molar fraction, which corresponds to about $7.6 \%$ in the hydrophobic region of the bilayer. The crystallographic structure of SecYEG (PDB ID:3DIN) [33] was used to generate the scattering length density (SLD) profile associated with the embedded protein. The orientation of the SecYEG in the membrane was taken to be equally distributed with the cytoplasmic protrusion exposed to the bulk solution (distal to the $\mathrm{SiO}_{x}$ surface) in $50 \%$ of total occurrences [7]. For small bilayer-substrate separations, the submembrane space was too small to accommodate the extension of the SecYEG protein; in this region, the crystallographic SecYEG structure was truncated at the headgroup surface and evenly reallocated in the submembrane space so as to preserve the total protein volume. Peripheral SecA was modeled as a constant area fraction of protein extending from the headgroup surface. 

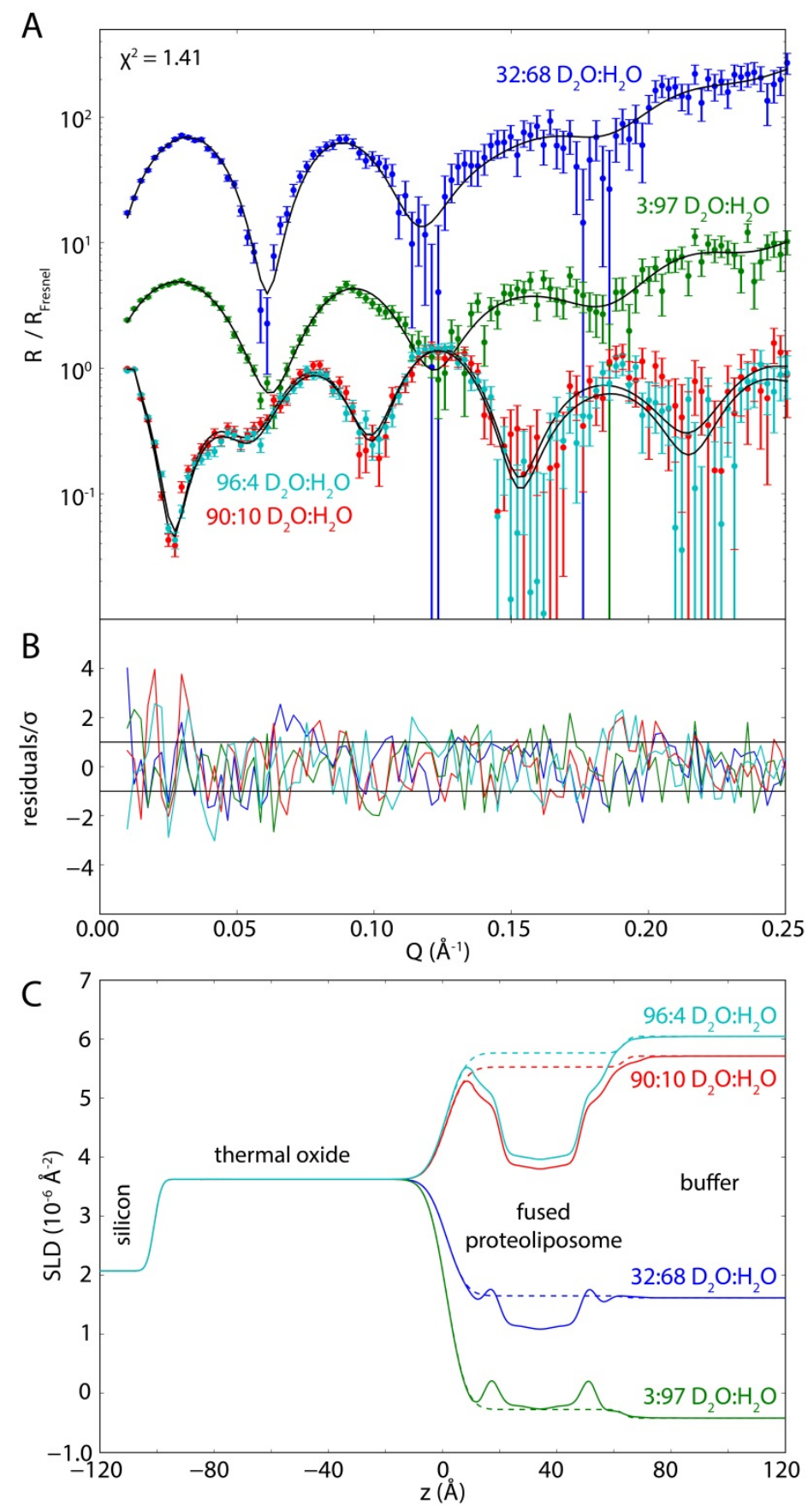

Figure 30: (a) Neutron reflectivity in buffers based on $\mathrm{D}_{2} \mathrm{O}, \mathrm{H}_{2} \mathrm{O}$. Multiple different mixtures of $\mathrm{D}_{2} \mathrm{O} / \mathrm{H}_{2} \mathrm{O}$ are shown. Reflectivity was normalized to the Fresnel reflectivity expected from the interface between silicon and each buffer. Error bars represented $68 \%$ confidence intervals arising from Poisson counting uncertainty. (b) Residuals of volume occupancy modeled optimization to reflectivity curves. (c) SLD profiles derived from the optimized volume occupancy model. Solid and dashed lines showed the SLD profiles of two incoherently added models corresponding to surface regions with and without adsorbed proteoliposomes, respectively. 
Because of incomplete coverage of the proteoliposomes on the $\mathrm{SiO}_{x}$ surface, the data were modeled as the incoherent sum of the bilayer model and a second model in which significant areas $(>10 \mu \mathrm{m})[75]$ of the substrate contain no liposomes. Nonspecifically adsorbed protein, which is likely to be SecA not bound to SecYEG, was modeled as a constant area fraction and density extending into the solution from the substrate surface. The fraction of the complete data set to be described by each model was allowed to vary in the optimizations. The results of the model optimization (Figure 30a, solid lines) achieved a reduced $\chi^{2}$ value of 1.41; the residuals are shown (Figure 30b). The SLD profiles corresponding to the two model regions (with and without the SecYEG-containing bilayer) are shown as solid and dashed (Figure 30c). Note that at distances from the substrate in which water is present, the SLD profile changes with the deuteration level of the buffer.

The individual molecular components are shown in Figure 31. The top $43 \%$ of the plot (Figure 31a, dashed lines) shows the structure of model region without proteoliposomes; the only molecular component is the nonspecifically adsorbed surface protein. The bottom $57 \%$ is the bilayer-containing region of the model (Figure 31b, solid lines), showing the two bilayer leaflets, SecYEG, and SecA. Complete list of fitting parameters and their optimized values is provided (Table 3). A detailed view of components at the bilayer/ surface interface is shown (Figure 31c). A principal quantity of relevance to this work is the average height of the submembrane space separating the $\mathrm{SiO}_{x}$ substrate from the headgroups of the substrate-proximal lipid leaflet, $9.1_{-0.4}^{+0.5} \AA$. More precisely this is defined as the distance from the center of the oxide/water interface to the center of the choline/water interface. Though relatively small, this amount of space is sufficient to accommodate precursor proteins. Unfolded protein segments exhibit very low bending stiffness. In particular, the submembrane space is more than double the $3.9 \AA$ persistence length of an unfolded polypeptide, 
which itself is about the same as the size of a single amino acid, $\sim 4 \AA$ [76]. Additionally, $9.1 \AA$ is equivalent to the radius of gyration of an approximately 10 amino acid long unfolded polypeptide (random coil) in free solution [77]. Other values of interest from the NR analysis include the total thickness of the bilayer (including headgroups), $48.5_{-0.9}^{+1.0} \AA$; the total separation of the top surface of the bilayer from the substrate, $57.5_{-0.7}^{+1.0} \AA$; and the total bilayer coverage, $15.9_{-0.6}^{+0.7} \%$. This last quantity is smaller than the bilayer coverage observed in AFM measurements on borosilicate glass. This could be due to topographical or surface chemical differences that alter proteoliposome spreading [78]. 

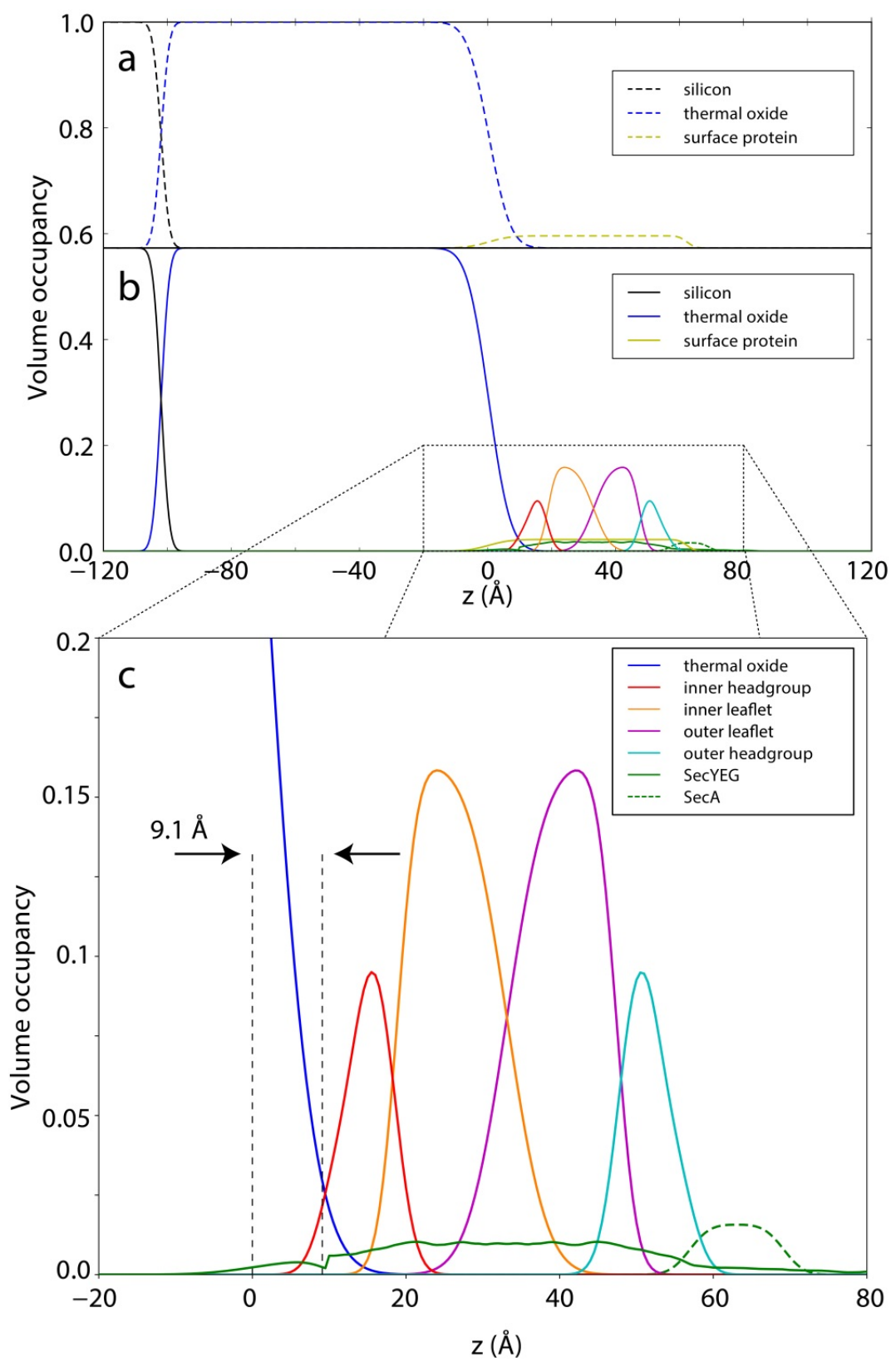

Figure 31: (a,b) Results of the optimized underlying volume-occupancy model. Solid and dashed lines showed the two model regions with and without adsorbed proteoliposomes, respectively. The solid line at 0.57 fractional occupancy reflected the relative weight of the two models determined in the optimizations. (c) Detailed view of the model in the vicinity of the $\mathrm{SiO}_{x}$ surface showing the $9.1 \AA$ submembrane space. 
Table 3: Neutron reflectometry fitting data. List of fit parameters with optimization limits and optimized values with confidence intervals (CI) [69]

\begin{tabular}{|c|c|c|c|c|c|}
\hline Parameter (units) & $\begin{array}{l}\text { Lower } \\
\text { bound }\end{array}$ & $\begin{array}{l}\text { Upper } \\
\text { bound }\end{array}$ & $\begin{array}{l}\text { Mean } \\
\text { value }\end{array}$ & $\begin{array}{l}\text { Lower } \\
68 \% \\
\text { CI }\end{array}$ & $\begin{array}{l}\text { Upper } \\
68 \% \\
\text { CI }\end{array}$ \\
\hline \multicolumn{6}{|l|}{ Beam parameters } \\
\hline Total reflectance & 0.9 & 1.05 & 0.983 & 0.977 & 0.988 \\
\hline Sample broadening factor & 0.1 & 4.0 & 0.16 & 0.12 & 0.25 \\
\hline Angle misalignment $\left(^{\circ}\right)$ & -0.005 & 0.005 & 0.0014 & -0.0014 & 0.0040 \\
\hline \multicolumn{6}{|l|}{ Bulk parameters } \\
\hline SLD of first data set $\left(10^{-6} \AA^{-2}\right)$ & 4.0 & 6.4 & 5.66 & 5.59 & 5.72 \\
\hline SLD of second data set $\left(10^{-6} \AA^{-2}\right)$ & -0.566 & 2.4 & 1.65 & 1.60 & 1.69 \\
\hline SLD of third data set $\left(10^{-6} \AA^{-2}\right)$ & 4.0 & 6.4 & 5.99 & 5.92 & 6.05 \\
\hline SLD of fourth data set $\left(10^{-6} \AA^{-2}\right)$ & -0.566 & 2.4 & -0.33 & -0.41 & -0.26 \\
\hline Background of first data set $\left(10^{-6}\right)$ & -10 & 100 & 0.30 & 0.23 & 0.37 \\
\hline Background of second data set $\left(10^{-6}\right)$ & -10 & 100 & 1.98 & 1.89 & 2.08 \\
\hline Background of third data set $\left(10^{-6}\right)$ & -10 & 100 & 0.18 & 0.11 & 0.24 \\
\hline Background of fourth data set $\left(10^{-6}\right)$ & -10 & 100 & 2.34 & 2.18 & 2.50 \\
\hline \multicolumn{6}{|l|}{ Substrate parameters } \\
\hline $\begin{array}{l}\mathrm{Si} / \mathrm{SiO}_{x} \text { rms interfacial roughness } \\
(\AA)\end{array}$ & 2 & 15 & 2.19 & 2.05 & 2.45 \\
\hline $\mathrm{SiO}_{x}$ thickness $(\AA)$ & 70 & 110 & 102.62 & 102.38 & 102.85 \\
\hline $\mathrm{SiO}_{x} \operatorname{SLD}\left(10^{-6} \AA\right)$ & 2.4 & 4.4 & 3.168 & 3.598 & 3.639 \\
\hline $\mathrm{SiO}_{x}$ rms surface roughness $(\AA)$ & 2 & 25 & 4.53 & 3.99 & 5.00 \\
\hline \multicolumn{6}{|l|}{ Bilayer parameters } \\
\hline Substrate-headgroup separation $(\AA)$ & -5 & 50 & 9.10 & 8.66 & 9.58 \\
\hline Acyl chain length per leaflet $(\AA)$ & 10 & 25 & 13.25 & 12.85 & 13.72 \\
\hline Volume fraction of bilayer & 0 & 1 & 0.278 & 0.269 & 0.287 \\
\hline Volume fraction of peripheral protein & 0 & 0.4 & 0.06 & 0.01 & 0.22 \\
\hline Length of peripheral protein $(\AA)$ & 10 & 80 & 15.5 & 10.6 & 72.2 \\
\hline $\begin{array}{l}\text { Volume fraction of peripheral protein } \\
\text { after rinsing }\end{array}$ & 0 & 0.4 & 0.03 & 0.01 & 0.08 \\
\hline \multicolumn{6}{|l|}{ Nonspecific adsorption parameters } \\
\hline $\begin{array}{l}\text { Volume fraction of nonspecifically } \\
\text { adsorbed protein }\end{array}$ & 0 & 0.4 & 0.056 & 0.045 & 0.067 \\
\hline $\begin{array}{l}\text { Length of nonspecifcally adsorbed } \\
\text { protein }\end{array}$ & 10 & 80 & 57.5 & 53.4 & 60.3 \\
\hline $\begin{array}{l}\text { Volume fraction of nonspecifically } \\
\text { adsorbed protein after rinsing }\end{array}$ & 0 & 0.4 & 0.065 & 0.055 & 0.074 \\
\hline $\begin{array}{l}\text { Fraction of surface described only by } \\
\text { nonspecific adsorption }\end{array}$ & 0 & 1 & 0.43 & 0.40 & 0.45 \\
\hline
\end{tabular}




\section{Membrane surface characterization via Atomic Force Microscopy}

\subsection{Single molecule observation of nucleotide induced con- formational changes in basal SecA-ATP hydrolysis}

We shed light on the conformational dynamics of SecA during ATP hydrolysis by imaging single molecules in near-native conditions [24]. Following previous work [6-8], proteins were adsorbed to freshly cleaved mica and imaged via tapping mode AFM in aqueous solution. Salient topographic information, including the height and area of individual macromolecules, was extracted algorithmically and collected for statistical analyses [79]. We studied first wild-type SecA (SecA-WT). SecA-WT exists in equilibrium between monomer and dimer in E. coli. We focused on conformational dynamics of SecA monomer by choosing solution conditions that bias the system away from dimer [80]. We determined the relationship between specific topographical populations and the functionally important protein binding domain, PBD, of SecA. The distribution of feature height for individual SecA-WT molecules in the apo state (i.e., not exposed to any nucleotide after purification) is presented (Figure 32a, black). These data show that most of the conformations exhibit a maximum height of around $4 \mathrm{~nm}$. A closer inspection reveals a minimum separating two subpopulations that are spaced $\sim 0.5 \mathrm{~nm}$ apart. Although small, this separation is above the $\sim 0.1 \mathrm{~nm}$ vertical resolution of the technique [17]. We observed an additional subpopulation in the height distribution as a distinct shoulder at about $5.1 \mathrm{~nm}$ (Figure 32b, light blue shade). This shoulder population was substantial, comprising $29 \%$ of the total. When a variant of SecA with the PBD deleted (SecA $\triangle \mathrm{PBD}$ ) was analyzed, the population at $5.1 \mathrm{~nm}$ was diminished significantly (Figure 32a, light blue shade). At the same 
time, the population at $4.3 \mathrm{~nm}$ was enhanced by roughly the same amount (compare Figure $32 \mathrm{a}$ and b, green shades), and the other three populations were essentially unchanged compared to the WT enzyme (Figure 32a and b; dark blue, orange, and pink shades). We emphasize that the peak positions of the two major populations match exactly; hence, SecA-WT and the mutant lacking the PBD are likely to be in the same overall orientation on the mica. Hence, the conformational state at 5.1 nm appears to be directly related to the PBD. We note that cells require the PBD of SecA; SecA $\triangle \mathrm{PBD}$ was overproduced, and the purified sample contained $<10 \%$ residual SecA-WT.

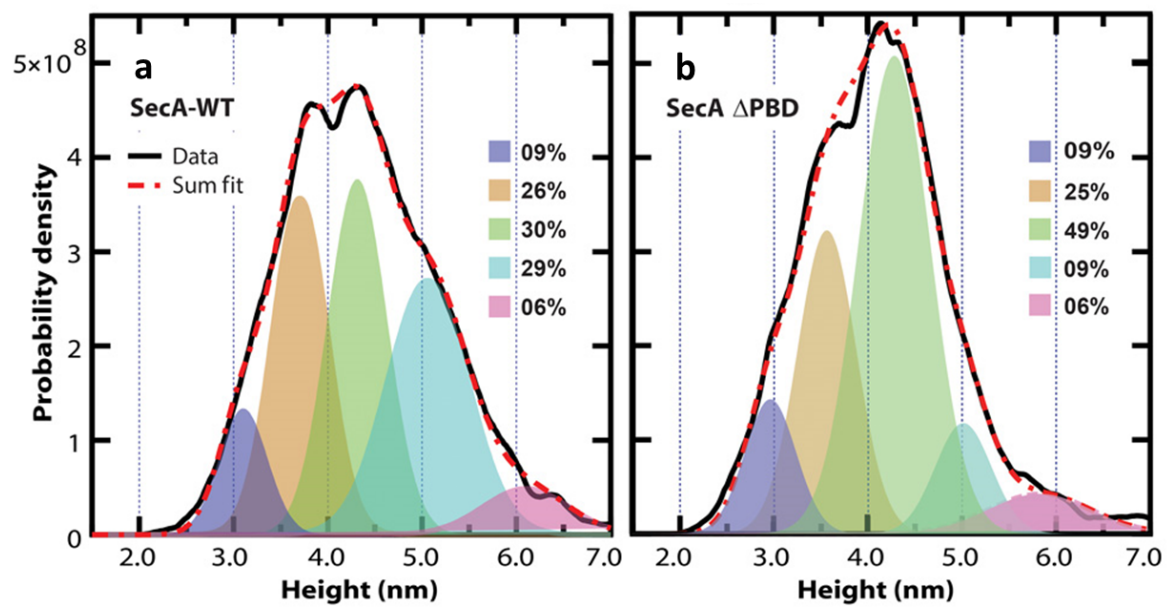

Figure 32: (a) Height distribution of SecA-WT (black solid line; number of different complexes that were measured $\mathrm{N}=3726$ ) showing multiple subpopulations. Five Gaussian fits with relative weights and the sum thereof (red dashed) are also shown. (b) Analogous data and fitting for the mutant SecA $\triangle$ PBD (experiment: black, $\mathrm{N}=$ 4009; sum fit: red dashed. Note the population reduction of the population at 5.1 $\mathrm{nm}$ in the case of the mutant. We chose to fit to five Gaussians because this number coincides with the number of prominent convex regions (populations) within the WT distribution; this number led to coefficient of determination $\left(\mathrm{R}^{2}\right)$ values of $>0.997$ for both SecA-WT and SecA $\triangle \mathrm{PBD}$.

Before characterizing enzyme topography during ATP hydrolysis, we sought to verify the ATP hydrolysis activity of SecA samples as discussed in chapter 2.1. After confirming that ATPase activity was maintained, we studied the dynamic topography 
of SecA during ATP hydrolysis using our AFM. The SecA-WT and the SecA $\Delta$ PBD mutant were subjected to ATP on the surface. The concentration of ATP chosen for this study was significantly higher than the reported affinity for ATP $\left(\mathrm{K}_{d} \sim 300\right.$ nM) [81]. The height distributions indicated that SecA-WT was conformationally more dynamic as compared to the SecA $\triangle \mathrm{PBD}$ mutant. Figure 33 (a and b) shows height distributions of SecA-WT and SecA $\triangle \mathrm{PBD}$, respectively. The full width at half maximum (FWHM) of SecA-WT heights was larger than that of the mutant. The FWHM in the presence of ATP was $2.3 \mathrm{~nm}$ for SecA-WT compared to $1.5 \mathrm{~nm}$ for the SecA $\triangle \mathrm{PBD}$ mutant. This difference can be attributed to the PBD and motions thereof.
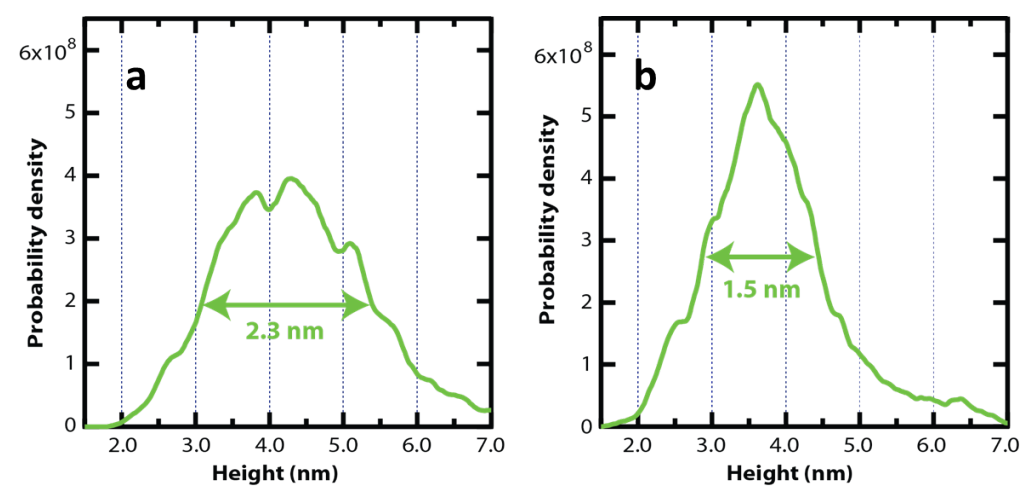

Figure 33: (a) Height distribution of SecA-WT (b) Height distribution of SecA $\triangle$ PBD. FWHM of both distributions are shown on the curves. SecA-WT examines a wider FWHM than that of the mutant indicating higher conformational dynamics due to the presence of the protein binding domain.

We followed individual SecA-WT molecules in real time and directly visualized conformational dynamics during ATP hydrolysis. To better observe conformational dynamics, we enhanced the temporal resolution to $\sim 100 \mathrm{~ms}$ by repeatedly scanning in one dimension to generate kymographs. First, a traditional two-dimensional image was taken to identify a protein of interest (Figure 34A). Then, the slow scan axis was disabled to repeatedly scan over the same protein to monitor conformational dynamics under physiological conditions in the absence of ATP (Figure 34B). We then 
exploited the large difference in the protein area footprint distributions to monitor real-time dynamics as the substrate ATP was consumed. Kymographs during ATP hydrolysis revealed at least two distinct conformational states exhibiting different width profiles that interconverted back and forth over time (Figure 34C; see also line scans in Figure 34D, which are separated by $\sim 100 \mathrm{~ms}$ ). This apparent conformational "flickering" was captured over extended periods, illustrating the reversible nature of the dynamics. Figure 34E illustrates a kymograph of SecA-WT in the presence of ATP over more than $75 \mathrm{~s}$. Here, the protein interconverted between a "compact" and an "extended" state. The green trace in Figure 34F shows the full width distribution profile of this molecule for the same period of time. The reversible switching of the molecule between compact and extended states is reflected in the histogram as two populations, which are separated by a minimum, positioned at $\sim 29 \mathrm{~nm}$. The data exhibit at least two conformational states, despite differing scan trajectories with respect to the adsorbed protein. Note that all width measurements are overestimates of true molecular dimensions due to tip convolution (nominal tip radius, $\sim 8 \mathrm{~nm}$ ). During ATP hydrolysis (i.e., with ATP present), the protein favored the extended state over the compact state. Specifically, it remained in the extended state for $84 \%$ of the time and $14 \%$ in the compact state. We note that the rate-limiting step of the SecA ATP hydrolysis cycle is ADP release [81]. Hence, the prominent extended state is likely to represent the ADP-bound post hydrolysis state of SecA. 

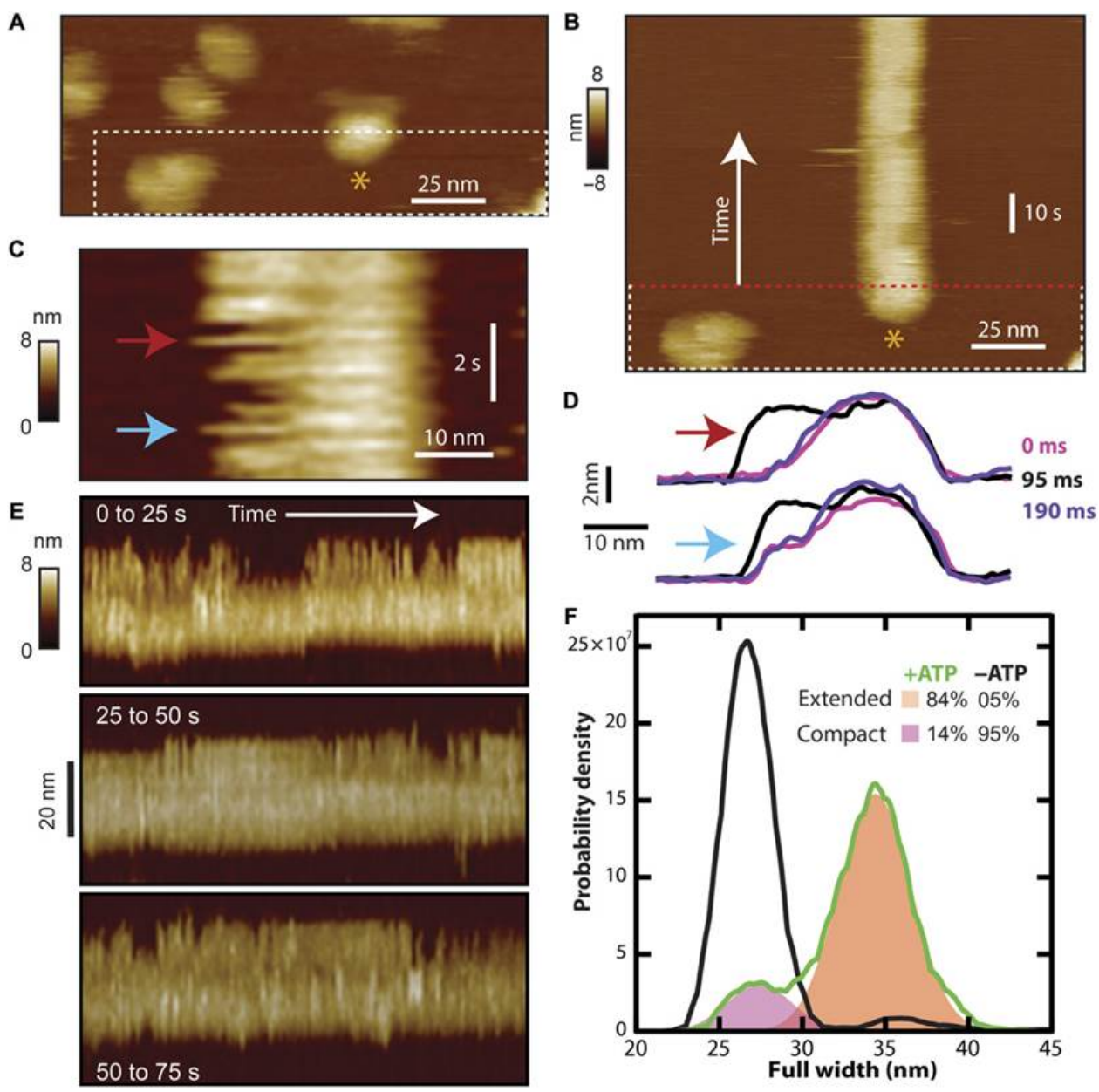

Figure 34: (A) AFM image of SecA-WT in the absence of ATP, white dashed rectangle shows area for detailed study, and asterisk identifies molecule of interest. (B) Upon rescanning and identifying the same molecule, a kymograph is initiated: the time axis begins at the top of the area (red dashed line). (C) Detailed view of kymograph in the presence of ATP. (D) Sequential height profiles separated by 95 ms (temporal sequence: pink, black, and purple) corresponding to arrows in (C). (E) Kymograph of SecA with ATP over $75 \mathrm{~s}$ and (F) histogram of AFM-measured SecA full width (number of scan lines, $N=406$ ) showing two subpopulations: compact and extended.

The height distribution for individual SecA-WT molecules in the apo state exhibited a maximum at around $4 \mathrm{~nm}$, in overall agreement with previously reported distributions for SecA when bound to SecYEG in membrane using the same technique $[7,8]$. AFM imaging indicated that the PBD explored a wide conformational 
space when SecA was exposed to ATP and provided evidence of PBD motion when compared SecA-WT to SecA $\triangle \mathrm{PBD}$. We followed individual SecA molecules in real time in an attempt to directly visualize conformational dynamics associated with ATP hydrolysis. To more readily observe these dynamics, we enhanced the temporal resolution by raster scanning in one spatial dimension rather than two. The resulting kymographs revealed two distinct conformational states of SecA: extended and compact. The conformational change from the compact state to extended state observed in the kymographs can be associated with ATP binding and hydrolysis. A conformational change is required for ADP release, and ADP release is the rate-limiting step [81]. Hence, the conformational change from the dominant extended state to the compact state is likely associated with ADP release. Previous studies have shown that only hydrolysable ATP can drive processive preprotein translocation but not ADP or other nonhydrolyzable ATP analogs [82,83]. Further, the PBD is known to be in direct contact with precursor proteins $[26,35,36]$. Therefore, the two dominant states observed in the kymographs are likely associated with translocation.

\subsection{Direct visualization of the $E$. coli Sec translocase engag- ing precursor proteins in lipid bilayers}

Here, we shed light on active translocase complexes [41]. Using a combination of biochemical experiments and AFM imaging, we studied the dynamic topography of the Sec translocase engaging two distinct precursors: one destined for the outer membrane [outer membrane protein A (pOmpA, alternatively known as proOmpA)] and the other destined for the periplasm [galactose-binding protein (pGBP)]. Our results suggested that active translocases exhibit conformations on the time scale of AFM imaging that vary with precursor species. We attribute the conformational differences 
to SecA-driven structural rearrangements within the translocase. Overall, the data suggest that the Sec translocation process works differently for different precursors.

\section{Translocase topography as a function of translocation stage and precursor species}

To define an initial state of the system, we allowed translocation of either pOmpA or pGBP to proceed for only $30 \mathrm{~s}$, at which point $\mathrm{ADP}-\mathrm{AlF}_{3}$ (ADPAlF) was added to halt further hydrolysis. The nonhydrolyzable ATP analog ADPAlF is thought to trap SecA in the transition state [84]. Immediately after ADPAlF addition, we deposited the reaction mix onto freshly cleaved mica. Samples were incubated, rinsed, and then imaged. All data were collected in imaging buffer using tapping mode at $\sim 32{ }^{\circ} \mathrm{C}$. Representative AFM images of the Sec translocase engaging precursors at different stages of translocation are displayed (Figure 35). The presence of lipid bilayers was confirmed by the observation of occasional defects (voids) that were $\sim 40 \AA$ deep from the surface of the upper leaflet of the bilayer [85]; one such void is indicated (Figure 35A, i, arrow). The images contained many punctate protrusions corresponding to translocases and translocons above the E. coli lipid bilayer. Representative individual protrusions are shown as insets at the right corner of each image in Figure 35A. To facilitate statistical analyses, salient topographic parameters were extracted and pooled together to construct kernel density estimates (smoothed histograms). On the basis of our previous work imaging SecYEG in lipid bilayers and SecYEG in complex with SecA in lipid bilayers $[6,8]$, many of the protrusions exhibited topography commensurate with the translocase [i.e., an approximate height range between 32 and $80 \AA$ with a major population at about $37 \AA$ [7]]. In the absence of SecYEG, SecA does not appear to bind the lipid bilayer in a preferred conformation [7]. Protrusions corresponding to the periplasmic side of SecYEG, which extend $<10 \AA$ above the membrane and do not engage SecA, were not included in the analysis [6]. In addi- 
tion, features exhibiting large heights $(>100 \AA)$, which were likely to be aggregates, were rare $(<15 \%$ of total) and not included in analysis. To evaluate protein crowding, a potential artifact, proteoliposomes with a fourfold lower protein-to-lipid ratio were prepared. Despite moderate variations expected from different sample preparations, the data suggest that crowding effects are negligible at the protein-to-lipid ratio used throughout this work $(\mathrm{P}: \mathrm{L}=1: 1000)$. 

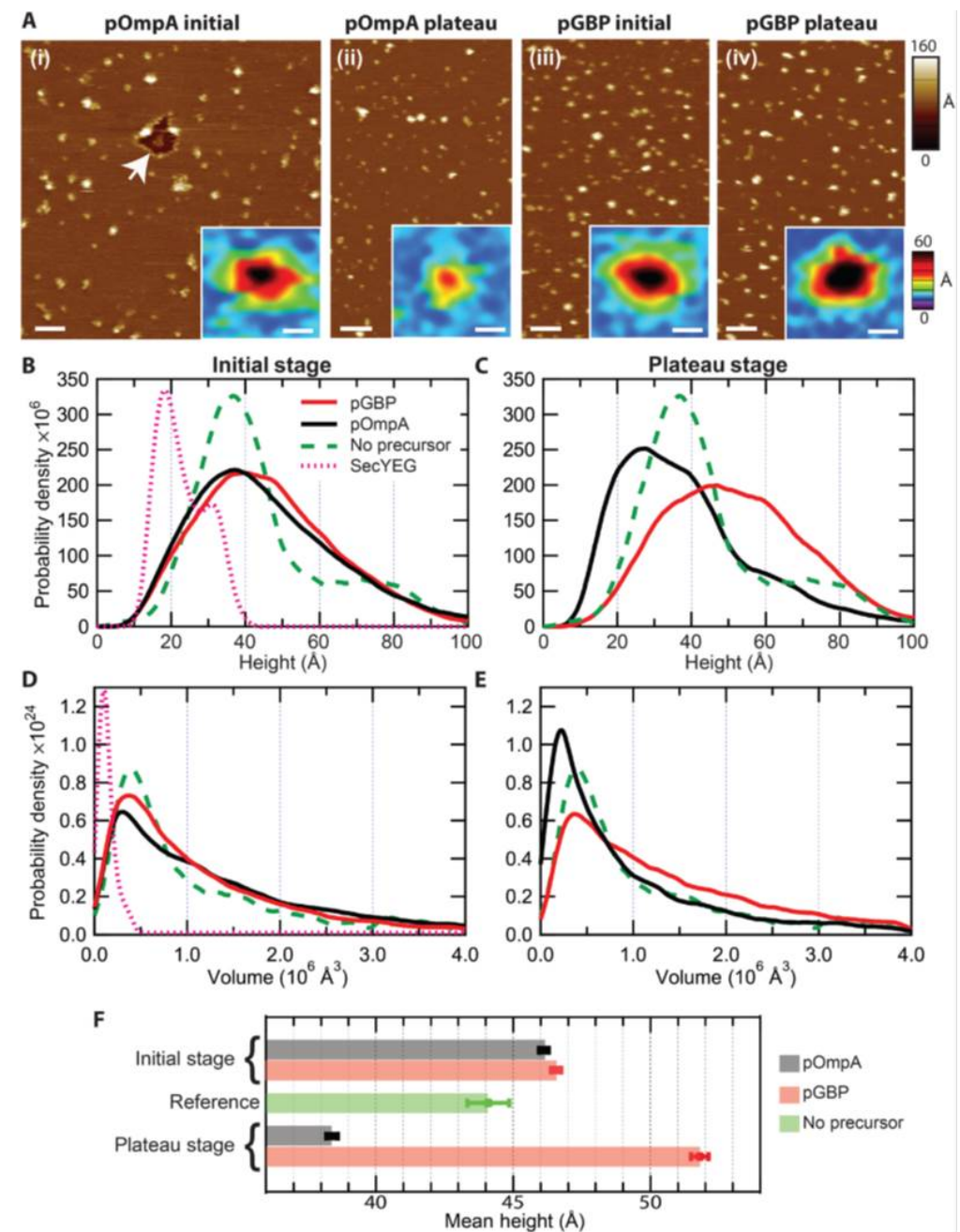

Figure 35: (A) AFM images of Sec translocases: (i) with pOmpA at the initial stage (30 s) of translocation, the arrow identifies a void in an otherwise continuous lipid bilayer; (ii) with pOmpA at the plateau stage of activity (3 min); (iii) with pGBP at $30 \mathrm{~s}$; and (iv) with pGBP at plateau (4.5 min). Lateral scale bars are $1000 \AA$ and the false color vertical scale spans $160 \AA$. Individual features are shown as insets with scale bars: $100 \AA$ (lateral) and $60 \AA$ (vertical). (B) Height and (D) volume distributions of active Sec translocases at $30 \mathrm{~s}$ with either pOmpA (red curve, number of features included, $\mathrm{N}=12,387$ ) or pGBP (black curve, $\mathrm{N}=10,063$ ). Data for translocases with no precursor (green dashed curve, $\mathrm{N}=587$ ) as well as SecYEG alone (magenta dotted curve, $\mathrm{N}=1875$ ) are shown for reference. After reaching the plateau stage of translocation activity, the $(\mathrm{C})$ height and $(\mathrm{E})$ volume distributions of translocases engaged with pOmpA (red curve, $\mathrm{N}=9565$ ) or with pGBP (black

curve, $\mathrm{N}=6592$ ) are shown. In all cases, translocation was halted by adding

ADPAlF at the prescribed times. Note that the vertical scales for SecYEG alone data were compressed two or fivefold in the height or volume plots, respectively. $(F)$ Arithmetic means of the height distributions. Error bars show SEM. 


\section{Topography of Sec $A_{2}$ in the translocase varies with precursor species}

To provide insight, we focused on heights of translocases in which SecA was in dimeric form, i.e., those exhibiting volumes within the range $0.9 \times 10^{6}$ to $2.0 \times 10^{6} \AA^{3}$. The resulting analyses with pGBP show that in the presence of ATP, translocases exhibited single-peaked height distributions (Figure 36a). In contrast, translocases engaging pOmpA exhibited multimodal height distributions under the same conditions (Figure 36b). To quantify these distinctions, we used Bayesian information criterion to determine the optimal number of model distributions to fit each dataset 37. This analysis prescribed one gamma distribution for pGBP in ATP. In contrast, three distributions were required for pOmpA subject to the same conditions. Gamma distributions were used because they naturally accommodate skewness inherent in the experimental data. In addition to the different number of modes, the peak locations for pGBP were shifted higher $(\sim 5 \AA)$ compared to pOmpA.
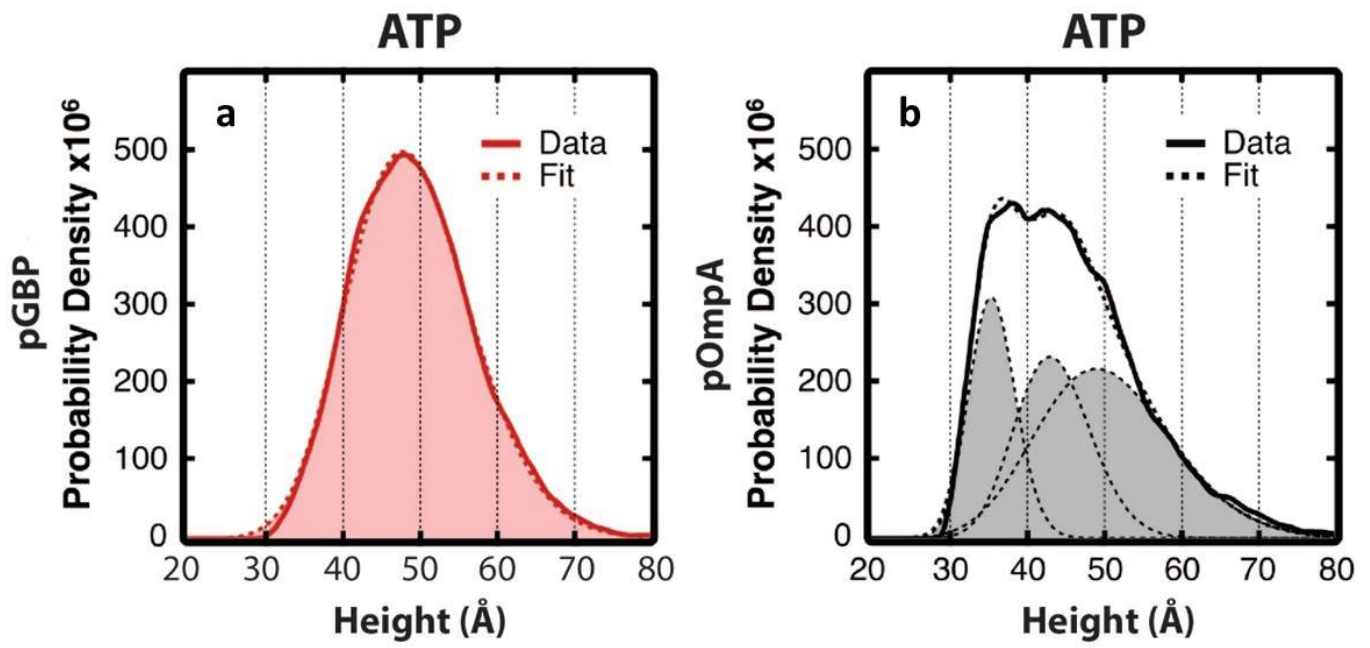

Figure 36: (a) Height distribution of translocase complexes on mica surface at plateau stage of pGBP. (b) Height distribution of translocase complexes on mica surface at plateau stage of pOmpA. 


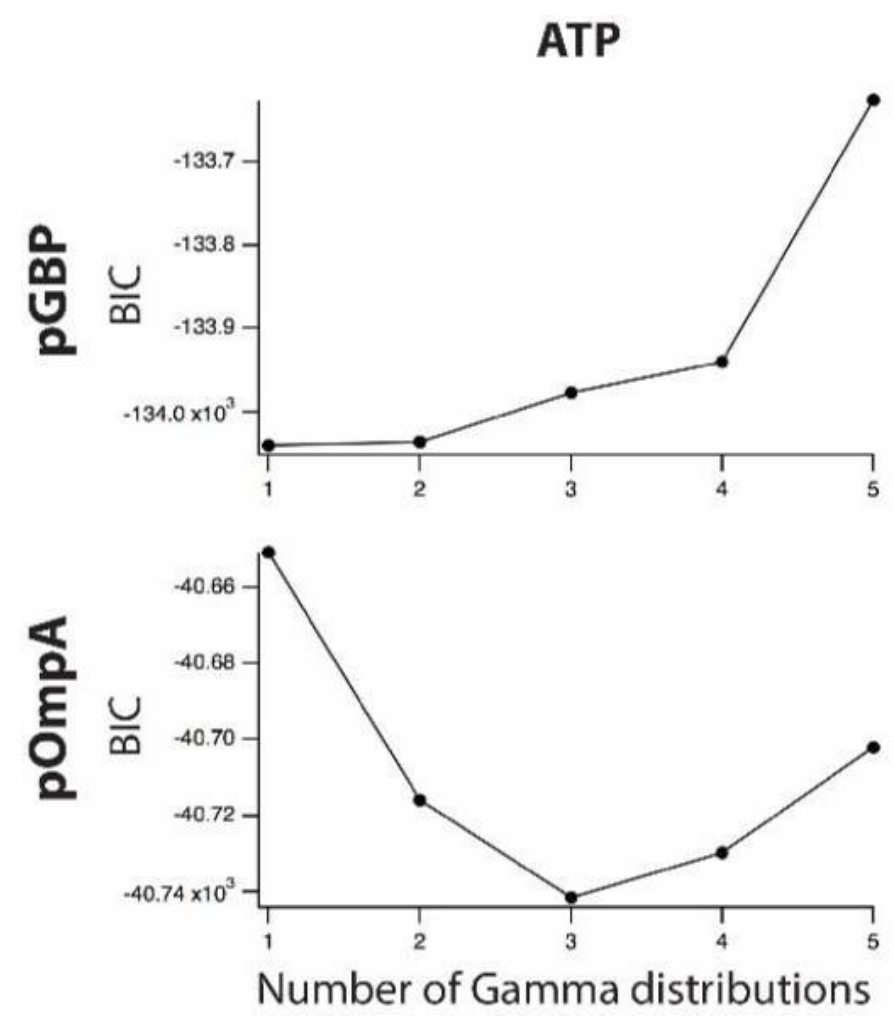

Figure 37: Bayesian information criterion (BIC) versus number of model Gamma distributions for each data set shown in Figure 36. The number of distributions corresponding to the BIC minimum was used to optimally fit the data.

We imaged translocases at work under near-native conditions. AFM data complement high-resolution structures by providing a direct view of the Sec translocase engaging precursor proteins in a lipid bilayer and in aqueous buffer solution. Experiments showed translocase complexes undergoing significant topographic changes in a manner that depended on precursor species. AFM imaging requires the biological material to be adsorbed onto a surface such as mica. This can prompt questions about biological activity. We showed in chapter 2.1 that the basal ATPase activity of surface-adsorbed SecA is similar to solution activity. We also found that Sec translocases embedded in lipid bilayers supported on mica achieve ATP-driven translocation with a turnover number $>1$ as discussed in chapter 3 . This is similar in magnitude to the turnover achieved in traditional translocation assays [46] and supports the no- 
tion that the visualization provided by AFM reflects active translocase conformations.

AFM measurements provide topographical corroboration for the significant precursordependent differences recently observed in the translocation reaction, including in the apparent rate constant and in the extent of precursor translocation [46]. One interpretation of the AFM data is that $\mathrm{pGBP}$ and $\mathrm{pOmpA}$ are transported across the cytoplasmic membrane by mechanisms that are distinct. However, there remains the alternative possibility that all the mechanical steps required for translocation are the same for both precursors, but differing kinetics gives rise to our observations. For example, during hydrolysis, the system could spend substantially more time in one conformation when engaging pGBP compared to pOmpA. Note that from the in-solution measurement, $\mathrm{k}$ for $\mathrm{pGBP}$ is lower than for $\mathrm{pOmpA}$, which means that the pGBP dwell time is longer. Further work with higher time resolution will be required to distinguish between these possibilities. In addition, it will be important to determine what physical property (or properties) of the precursor species is (are) responsible for the observed differences in the translocation process. Last, we point out that our AFM experiments on stalled complexes at coarsely separated translocation stages were not designed to discriminate between translocation step models such as the "push and slide" mechanism and the Brownian ratchet. Future studies using continuous monitoring of individual translocases over time may provide insight into this question. Overall, we report here the visualization of translocation machinery at work in near-native lipid bilayers. Our comparison of translocases engaging two different precursors side by side offers a direct view of this complex and dynamic process. Looking toward the future, high spatial-temporal precision single-molecule techniques are poised to shed further light on mechanisms underlying Sec system function. 


\section{Additional Projects}

\subsection{Force spectroscopy of SecA2-11 with Focused Ion Beam (FIB) modified AFM cantilever}

Force spectroscopy is a single molecule method suitable for measuring molecular interactions and structural energetics such as peptide-lipid interactions, protein folding, unfolding [86,87], etc. SecA, as discussed earlier, is a key component in cellular protein export which transports precursor proteins through the cytoplasmic membrane of E. coli using the energy released via hydrolysis of ATP. It exists both associated with the translocon YEG and free in the cytosol. The N-terminal region of SecA penetrates the membrane and lies at the interface of the hydrophobic and hydrophilic region of the membrane bilayer [33,88,89]. Specifically, the extreme amino terminal residues are critical for the translocation of proteins in E. coli. Work in the Randall group and others has shown that deletion of the first 10-20 amino acids of SecA results in a drastic reduction in translocation. Interestingly, if these residues are exchanged for a generic lipid tethering motif, the activity can be completely restored [36]. Motivated by these observations, we explored this peptide-lipid interaction using single molecule techniques [7,36]. The energy landscape governing the peptide-lipid interaction can be reconstructed and characterized once the force is measured. In previous work, our group used AFM to measure the force associated with the extreme N-terminal 10 amino acids of SecA (SecA2-11) interacting with a model lipid bilayer via force spectroscopy measurement $[31,90,91]$. Here, we study the same interaction but at higher temporal precision achieved through focused ion beam (FIB) modifications of cantilevers $[10,11,15,16]$. The essence of the high bandwidth strategy is to reduce the mechanical response time of the cantilever by lowering its mass. Ideally, this modification should be achieved without sacrificing the force preci- 
sion of the measurement (i.e. keeping the spring constant of the cantilever, $\mathrm{k}$, small). Higher bandwidth cantilevers offer the possibility of measuring short lived intermediate states, such as those that have recently been discovered in bacteriorhodopsin, a model membrane protein [18].

\section{Results}

Previous measurements with SecA2-11 showed that the interactions between this short peptide and a lipid bilayer were rather complicated, even with a single component zwitterionic (POPC) lipid species that was not native to the peptide. The histogram of the rupture force illustrated asymmetry. This led to two dissociation pathways, and two co-existing energy barriers were found. This discovery suggested the emergence of two principal states available for the peptides to occupy during the interaction with the lipid bilayer. In order to investigate this interaction in more detail, it became apparent that an improved cantilever that had increased temporal resolution was required. This modification of the cantilever was done with care and consideration of parameters such as the response time, the force stability, the force sensitivity, the damping regime, and the durability of the cantilever after modification. The ideal cantilever should be soft, able to rapidly respond to changes in force, precise, overdamped, and sustain the functionalization process. The secondary electron microscope (SEM) images of the cantilever before and after modification are shown in Figure 38.
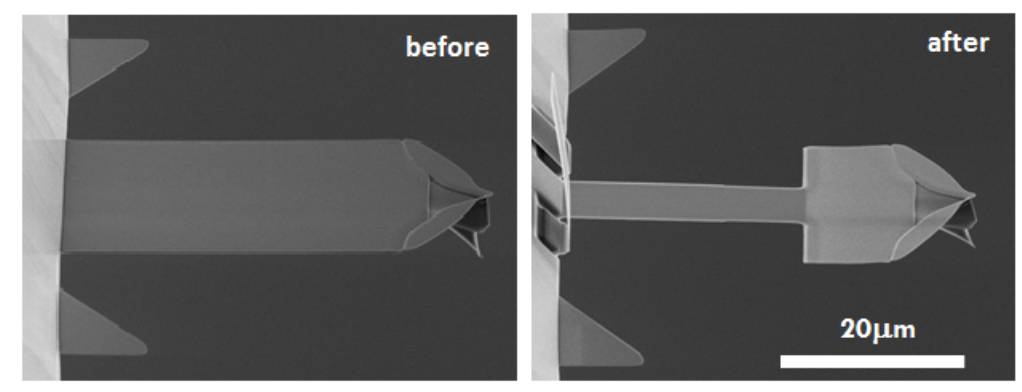

Figure 38: SEM image of the cantilever before and after the FIB modification. 
The modified cantilever was characterized by measuring the force power spectral density (PSD) and directly compared to the unmodified biolever mini and biolever long. This metric is valuable because it shows how the force noise is distributed in frequency space and can be used to read off the resonance frequency and evaluate the damping regimes. The biolever long cantilever is also commercially available, but due to its large size, is very sluggish (resonance frequency in fluid $\sim 1 \mathrm{kHz}$ ). Figure 39 shows PSD curves of the modified cantilever (black), the biolever mini (red), and the biolever long (blue) [15]. The PSD curve of the modified cantilever showed the suppressed resonance peak. This was an indication that the modified cantilever approached the desired overdamped regime. The overall reduction of force noise apparent in the modified cantilever also indicated improvement of force precision, and the removal of the metal coating layer was shown to improve a long term force stability of the cantilever [92]. The modified biolever mini outperformed the unmodified version and approached the performance of the biolever long cantilever, but with a significant enhancement in bandwidth. Further, the integrated force noise curves shown in Figure 40 indicated that the modified cantilever had sub-pN force sensitivity whereas the unmodified cantilever could not approach this sensitivity range.

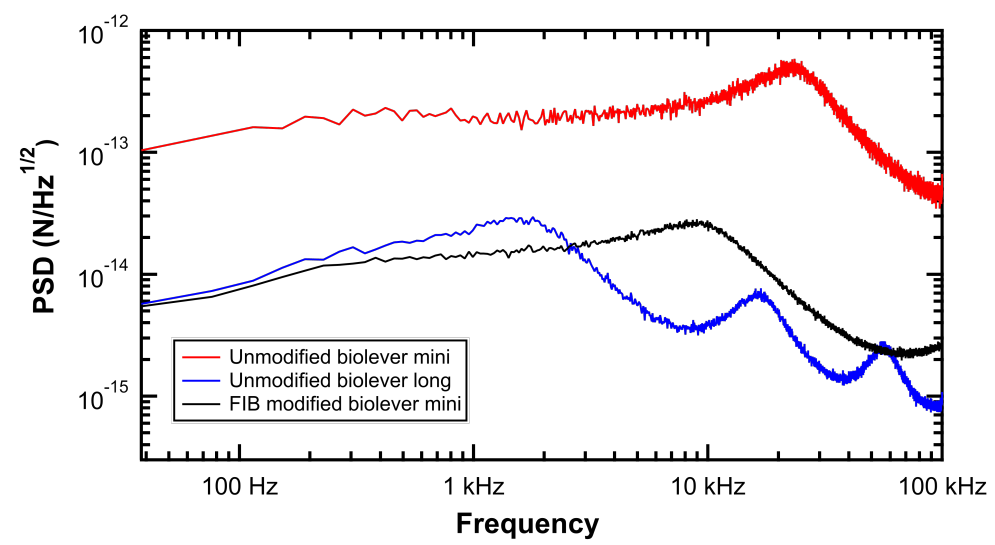

Figure 39: The force power spectral density graph of the modified biolever mini (black), the unmodified biolever mini (red) and the unmodified biolever long (blue). 


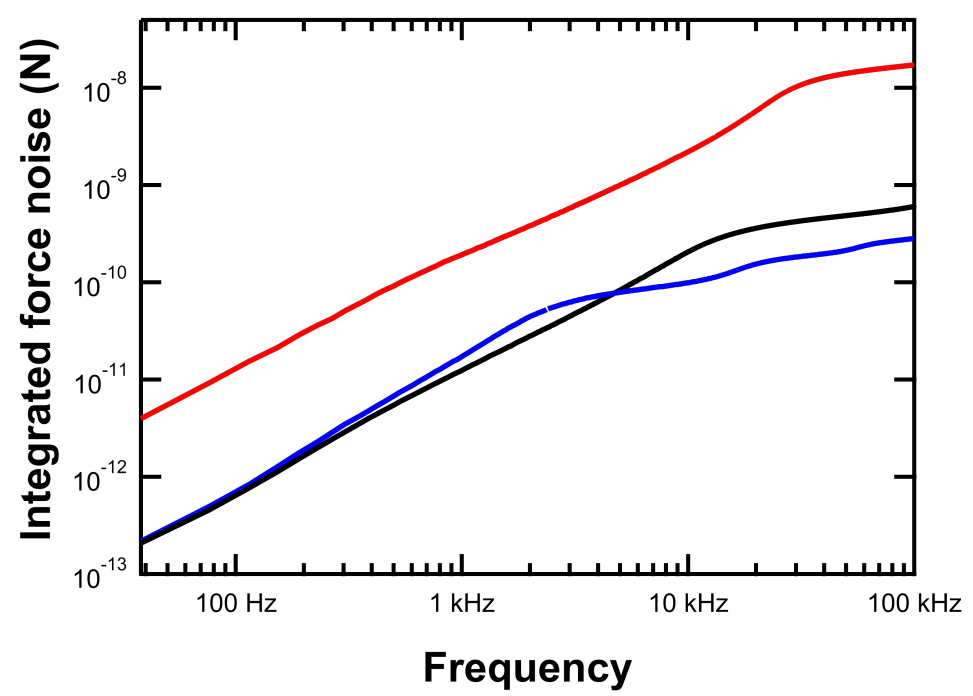

Figure 40: the integrated noise of the modified biolever mini (black), the unmodified biolever mini (red) and the unmodified biolever long (blue)

Not only did the modified cantilever show an increase in stability and sensitivity, it also showed faster response time, consistent with previous work in the field [15]. In conclusion, the modified cantilever performed better than both unmodified commercially available cantilevers. It had increased temporal resolution, fast response, sub-pN force precision over a $100 \mathrm{~Hz}$ bandwidth, long term force stability, low desired spring constant $(\mathrm{k}=8 \mathrm{pN} / \mathrm{nm})$, and was overdamped. The modified cantilever was therefore an ideal candidate for force spectroscopy measurements to observe the intermediate states of SecA2-11-lipid interactions. 


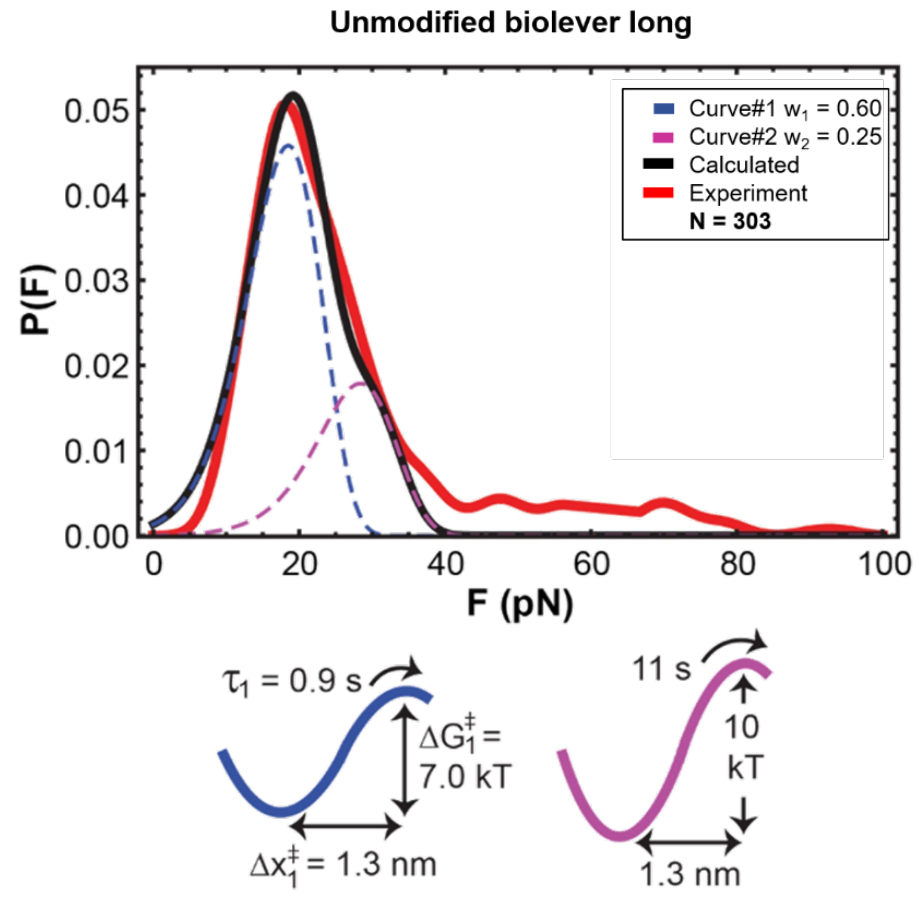

Figure 41: Force histogram obtained from biolever long cantilever

Figure 41 displays a histogram of rupture force during the retraction of SecA211 away from a POPC lipid bilayer with the biolever long cantilever, where the red solid line indicates the experimental results $[31,90,91]$. The black solid line in the plot represents the summation of the modeled results, and the calculated energy landscape comprised of two distinct energy barriers, indicated by the blue and pink dashed lines. The modeling was performed in collaboration with Prof. Ioan Kosztin's group (MU Physics). [31,90,91]. One thing to note is that each barrier is associated with a different weight. The calculated parameters correspond to the height of the energy barriers $\left(\Delta G_{i}^{\ddagger}\right)$, the distances to the transition states $\left(\Delta x_{i}^{\ddagger}\right)$, and the inverse of the off-rates in the absence of force $\left(\tau_{i}\right)$ which were determined for the interaction between the SecA2-11 peptide and a POPC lipid bilayer [31,90,91]. Force histograms obtained using the FIB modified biolever mini cantilever are shown in Figure 42. The data displays two prominent peaks at similar values as compared to the 
histogram obtained from measurements using unmodified biolever long cantilevers. The histogram's most prominent feature illustrates the signature of the interaction between SecA2-11 peptide and POPC lipid. One important conclusion is that the modified cantilever was able to sustain the functionalization process. The separation between the two peaks obtained from the modified cantilever also appeared to be more pronounced. This may be due to the increase in temporal resolution resulting from the modification described above. Additional interesting results came from the plot of the force as a function of time during which the peptide was interacting with the lipid bilayer. Figure 43 shows two examples of these results. Figure 43a shows the transition from a lower to a higher force state, and Figure 43b shows quasi-equilibrium 'flickering' events between these low and high force states. The transition and the flickering events suggest the emergence of intermediate states in the interaction of a SecA2-11 peptide and POPC lipid bilayer. It should also be noted that the separation of the low and high force states was approximately the separation of the two peaks needed to fit the force histogram. More experiments with the modified cantilever must be performed to further confirm the preliminary results discussed here. 


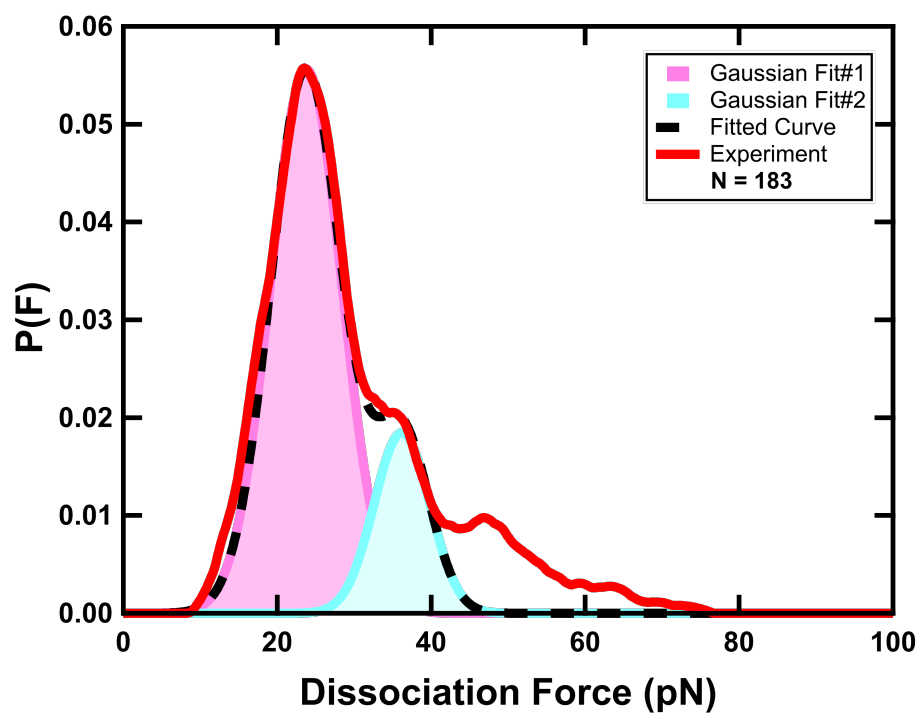

Figure 42: Rupture force distribution obtained from the modified cantilever
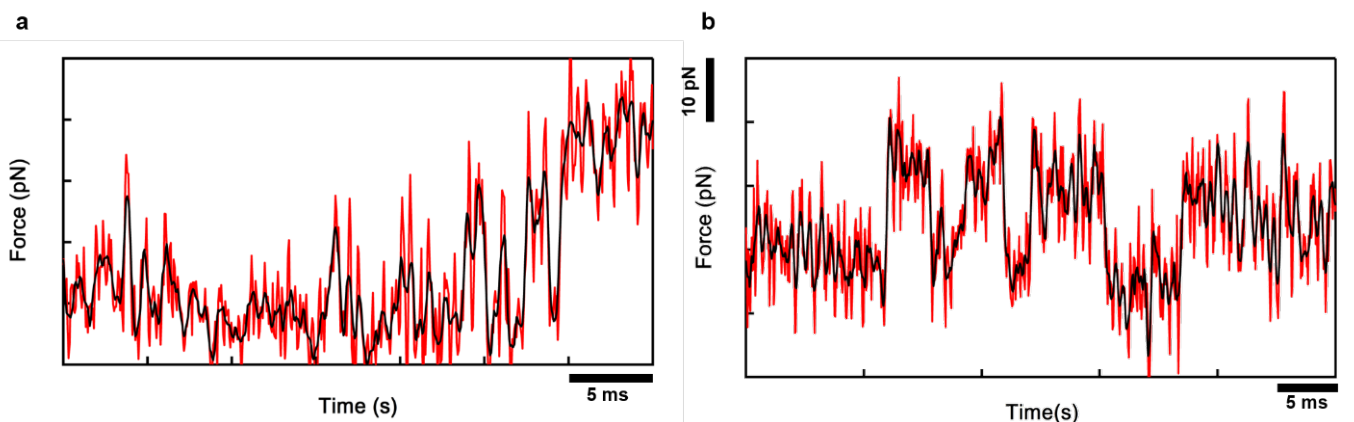

Figure 43: Zoomed-in views of the force as a function of time of SecA2-11 peptide and POPC lipid suggests the emergence of a complex multi-state interaction.

\subsection{Investigation of pyrophosphatase enzymes using AFM}

AFM is an ideal tool to probe conformational changes of macromolecules such as the pyrophosphatase enzyme in a near native environment (in fluid, at or near room temperature). However, parallel to the AFM investigation, the activity of the enzymes should also be verified, since the AFM measurement requires an underlying supporting surface. The activity assays performed on SecA showed that the SecA continued to hydrolyze ATP even when adsorbed to the mica support surface. Thus, AFM 
measurements could be analyzed with confidence that the biochemical activity was maintained. In this project, which is a collaboration between our group and Carlos Bustamante's group at U.C. Berkeley, the enzyme of interest was extracted from Streptococcus gordonii, and belongs to the family II inorganic pyrophosphatases, called Streptococcus gordonii inorganic pyrophosphatase, sgPP $[93,94]$. sgPP is an enzyme that assists with the conversion of one pyrophosphate into two inorganic phosphate molecules, which can be detected. The Bustamante group was interested in the diffusion behavior of enzymes such as sgPP. The core observation that triggered our collaboration was that many enzymes diffuse faster in the presence of their substrate [95]. Bustamante and co-workers developed a model based on chemo-acoustics to explain their data and we sought to probe this enzyme and model via direct AFM investigations.

Our group became interested in studying these enzymes from a mechanical AFM perspective - if an acoustic wave was generated by an enzyme undergoing catalytic turnover, perhaps we could measure the event using the AFM tip as a nanometer scale microphone. As a result of our initial investigations using the catalase enzyme, we developed an alternative hypothesis. In particular, we observed significant oligomeric state changes upon addition of substrate [25]. One would expect that this quaternary structure change would alter the diffusion behavior by lowering the drag force in a manner consistent with the Bustamante observations. To follow up on this alternative hypothesis, we studied a different enzyme that is a dimer in nature (as opposed to catalase which is tetrameric) and amenable to genetic modifications. Hence, in addition to the wild-type, sgPP was genetically modified in such a way that the two protomers that comprise one working unit of the enzyme were linked together in a single chain. The result of this enzyme engineering was the mutated species called a tandem dimer, which we named tdsgPP. The modification was done to assist 
the analysis of the AFM measurements and as an important control experiment in diffusion and other measurements carried out in the Bustamante laboratory. Since the two dimers are effectively tied together, oligomeric stage changes from one dimer to two monomers are forbidden.

\section{Results}

Surface based enzyme activity assay

The standard absorbance curve of sgPP enzyme activity is shown in Figure 44. The standard curve shows the absorbance values of the solution at $660 \mathrm{~nm}$ as a function of concentration of the product, which was the inorganic phosphate. The graph was fitted to a straight line and the value of the slope was calculated. This served as a platform for further calculations of the unknown product concentration obtained from the enzyme activity assay. For each measured value of the absorbance at $660 \mathrm{~nm}$ of the unknown, the concentration of the product can be calculated using the slope of this curve. concentrationofproduct $(\mu M)=\frac{\text { absorbanceat } 660 \mathrm{~nm}}{\text { slope }}$. The slope of the standard line in this case was $0.00067 \mathrm{unit} / \mu \mathrm{M}$.

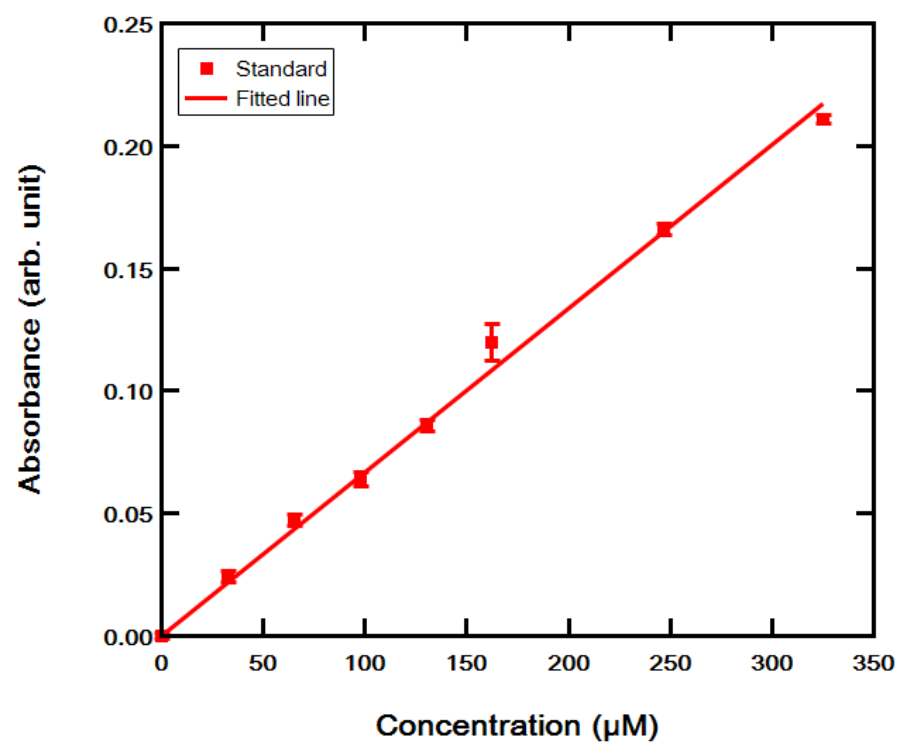

Figure 44: Standard curve of the activity of the sgPP enzyme 
The absorbance values at $660 \mathrm{~nm}$ of the sample from the no enzyme control, the solution control, the surface adsorbed wildtype enzyme and the tandem dimer enzymes were measured and converted to the product concentration according to the procedures discussed earlier. The solution control was acquired by sampling $40 \mu \mathrm{L}$ of the solution from mica surface right after the substrate was added. This control served as verification that the activity of the enzyme did not arise from the surface-adsorbed enzymes. The product concentration of each condition is plotted as a function of time and shown in Figure 45. The enzyme products, both wildtype and tandem dimers, were fitted with exponential rise functions, $y=y_{0}+A e^{-k x}$ where $\mathrm{y}_{0}$ was the $\mathrm{y}$ intercept of the curve, $\mathrm{A}$ was the amplitude, and $\mathrm{k}$ was the apparent rate constant of the enzyme reaction. The control plots were fitted with straight lines, $y=m x+c$. The graph clearly shows that the wildtype enzymes were more active than the tandem dimer enzymes, and the activity of the surface adsorbed enzyme for both wildtype and tandem dimer were above background level for both solution and no enzyme controls. For the wildtype enzyme, the fitted parameters were $\mathrm{y}_{0}=1263.2$ $\pm 46.5 \mu \mathrm{M}, \mathrm{A}=-1070 \pm 62 \mu \mathrm{M}$, and $\mathrm{k}=0.034 \pm 0.005 \mathrm{~min}^{-1}$. The tandem dimer fitted parameters were $\mathrm{y}_{0}=1800 \pm 515 \mu \mathrm{M}, \mathrm{A}=-1700 \pm 50 \mu \mathrm{M}$, and $\mathrm{k}=0.005 \pm$ $0.002 \mathrm{~min}^{-1}$. The solution control parameters were $\mathrm{m}=1.125 \pm 0.175 \mu \mathrm{M} / \mathrm{min}, \mathrm{c}=$ $68.831 \pm 14.1 \mu \mathrm{M}$. The no enzyme control parameters were $\mathrm{m}=0.1 \pm 0.1 \mu \mathrm{M} / \mathrm{min}$, $\mathrm{c}=67 \pm 10 \mu \mathrm{M}$. The small values of slopes for both controls ensure that the amount of product measured resulted from the surface adsorbed enzymes. Once the activities of the surface adsorbed enzymes were confirmed, the AFM experiments could be interpreted with confidence. 


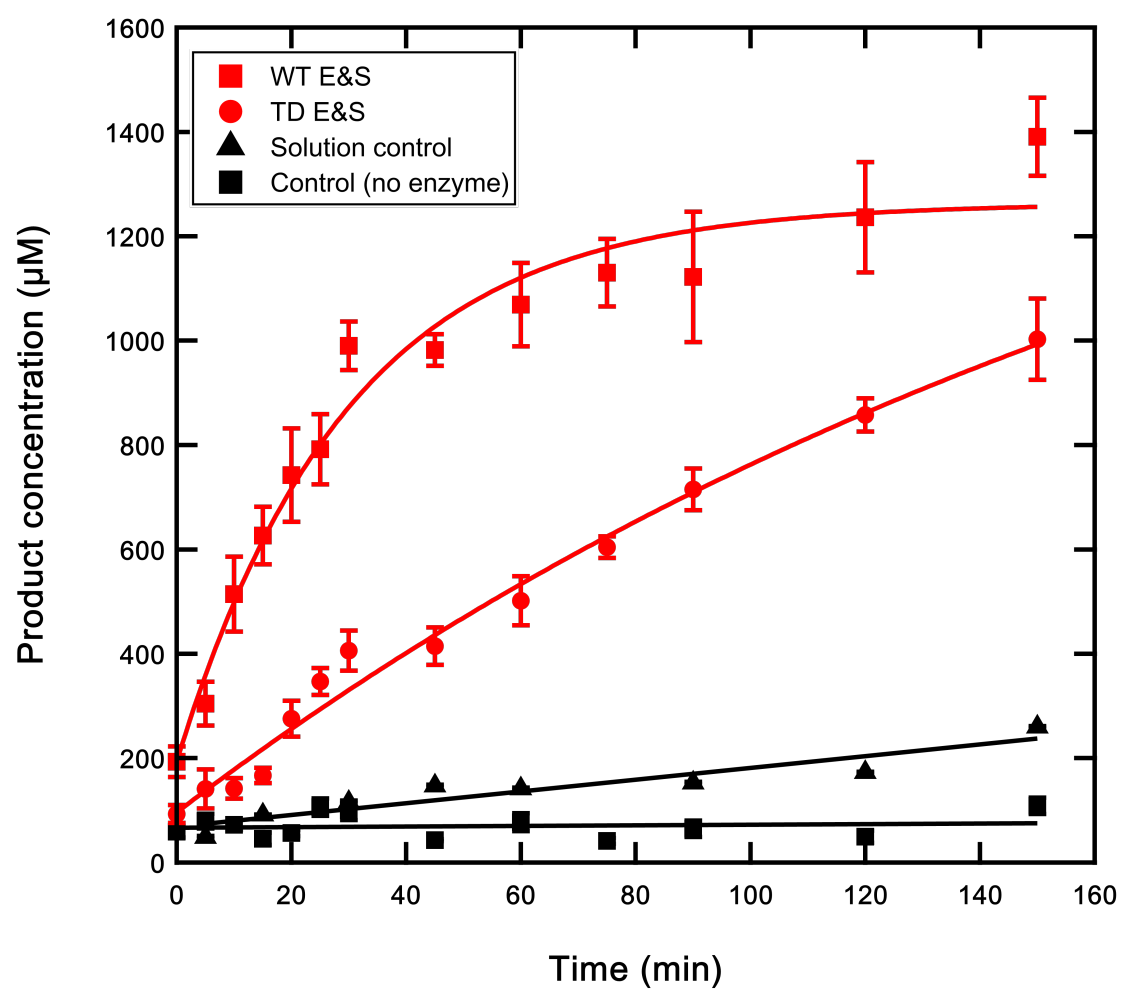

Figure 45: Enzyme activity plot showing the concentration of the product produced by surface adsorbed enzymes as a function of time for the wildtype (red, square), tandem dimer (red, circle), solution control, which was the solution taken from mica surface right after the substrate was added (black, triangle), and control without enzyme (black, square) 

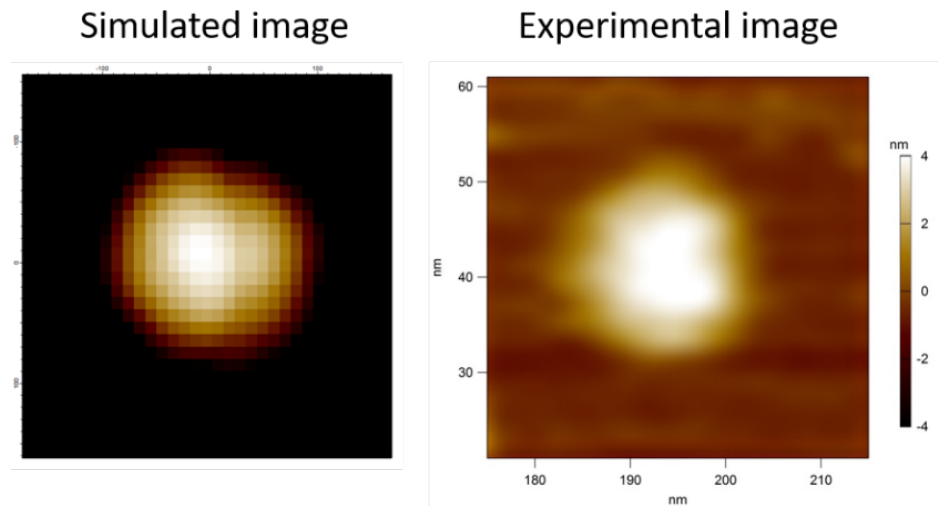

Figure 46: Comparison of simulated AFM image of the pyrophosphatase enzyme and an experimental image. To generate this simulation, we used the crystallographic coordinates of the enzyme (PDB code: 1K20) and a nominal tip radius of $8 \mathrm{~nm}$. We note that this simulated image comprises a dimer.

A simulated AFM image of the sgPP enzyme was carried out using the X-ray crystal structure of the enzyme from the protein data bank, 1K20. The custom Igor based program [79] generated an AFM image with assumed factory specified geometries of the AFM tip used in the actual measurement. The simulated image is shown in Figure 46 next to a cropped image of an actual individual enzyme for a direct comparison. Height, area, and volume values of the simulated enzyme were used as reference values for identification of individual enzymes and further analyses. The simulated values were height $\sim 4 \mathrm{~nm}$, area $\sim 300 \mathrm{~nm}^{2}$, and volume $\sim 670 \mathrm{~nm}^{3}$. The actual values of the height, area, and volume of the enzyme obtained from an AFM measurement were $\sim 3.8 \mathrm{~nm}, \sim 250 \mathrm{~nm}^{2}$, and $\sim 530 \mathrm{~nm}^{3}$, respectively. The direct comparison of the actual cropped particle, as shown in Figure 46, revealed the ability to detect a sgPP enzyme on the surface with AFM with confidence. Additional AFM images obtained are shown in Figure 47. The notations used were as described in the methods section: WT E and TD E for enzymes only, WT E+S and TD E+S for enzyme with substrate added after adsorption on mica surface. 


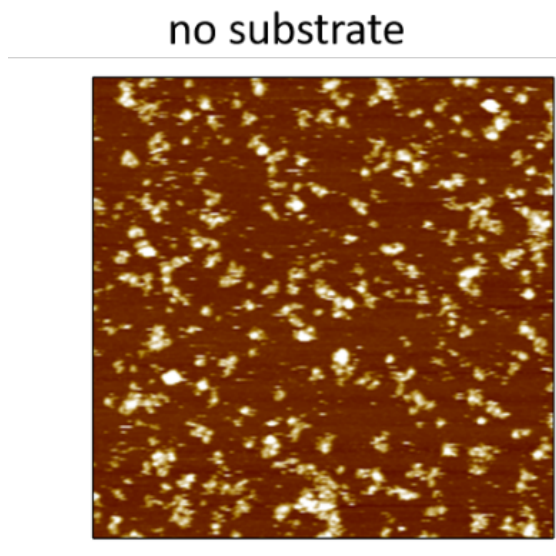

WT E

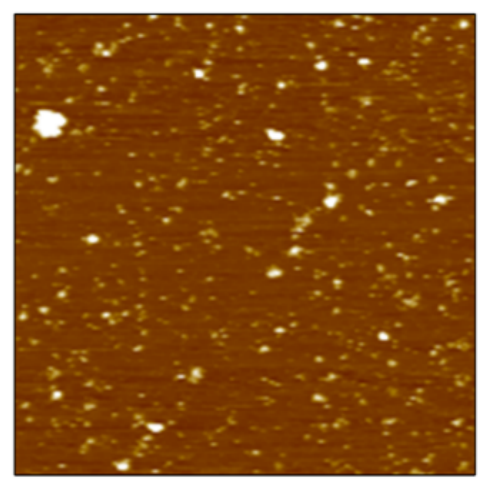

TD E activate on stage

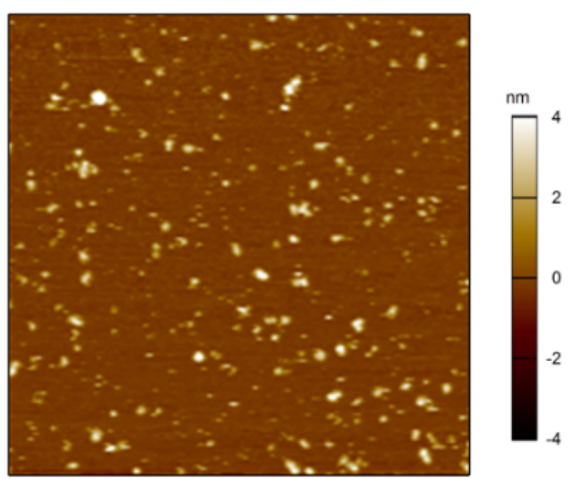

WT E+S

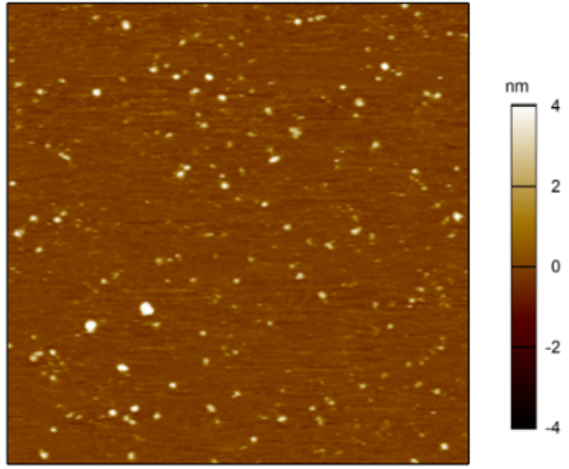

TD E+S

Figure 47: AFM images of the wild type enzymes (top) without substrate (left), with substrate added (right), and the tandem dimer enzymes (bottom) without substrate (left), with substrate added (right)

Histograms of volume, height, and area of the enzymes before and after exposing the substrates were extracted using the Hessian Blob algorithm [79]. A volume threshold of $250 \mathrm{~nm}^{3}$ was used to discard particles that were too small to be considered as full length enzymes, and hence likely degradation products. This value was set well below the expected value of an actual enzyme (from the crystal structure) in order to ensure that no enzymes were left out of analysis. The same threshold was used for both wild type and tandem dimer enzymes. The volume histograms of the wildtype enzyme with (black solid line) and without (red solid line) substrate 
are shown in Figure 48a. The volume histograms of the tandem dimer enzyme with (black dashed line) and without (red dashed line) substrate are shown in Figure 48b.
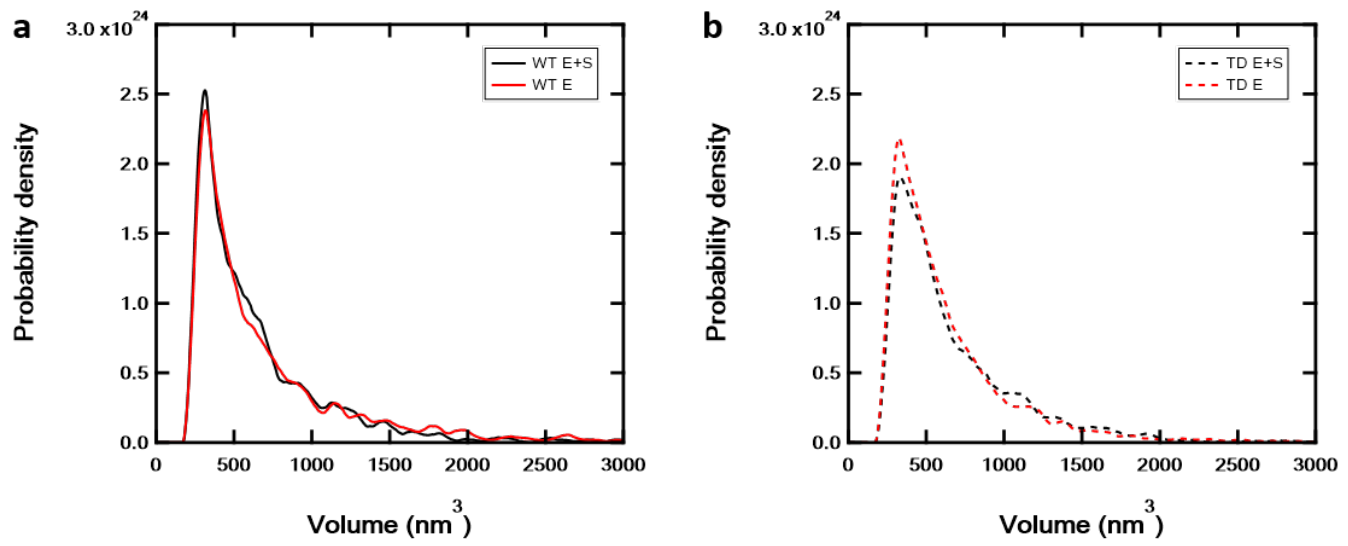

Figure 48: Volume histograms of the wild type enzyme with (red solid line) and without (black solid line) the presence of substrate (a) and the tandem dimer with (red dashed line) and without (black dashed line) the presence of substrate (b).

Height and area histograms of the wild type enzyme with (red) and without (black) substrate are shown in Figure 49a and 49b. The height and area histograms of the tandem dimer enzymes are shown in Figure 50a and 50b. The height histograms of the wildtype enzymes indicated that the enzymes exposed to the substrate showed a systematic decrease in height. The downward shift in height was approximately $5 \AA$. The area histograms of both wildtype and tandem dimer enzymes, with and without the substrate added, exhibited three major populations at $\sim 150 \mathrm{~nm}^{2}, \sim 300$ $\mathrm{nm}^{2}$, and $\sim 500 \mathrm{~nm}^{2}$, respectively. The first area peak value was so small that this may not belong to actual enzymes but rather to those that had been degraded. The reduction of the populations in the first area peak and the increase in the population in the second peak of the area histogram indicated reduction of the small material (potentially degraded enzymes) after exposure to the substrate. In the case of the wildtype enzymes, this is also shown in the AFM images by the fact that the mica surface appears to be cleaner after the substrate was added. The area peak position 
shifted slightly upward when the substrate was added in the case of the wildtype enzyme, but there was approximately no change in other area peak positions for the tandem dimer enzymes.
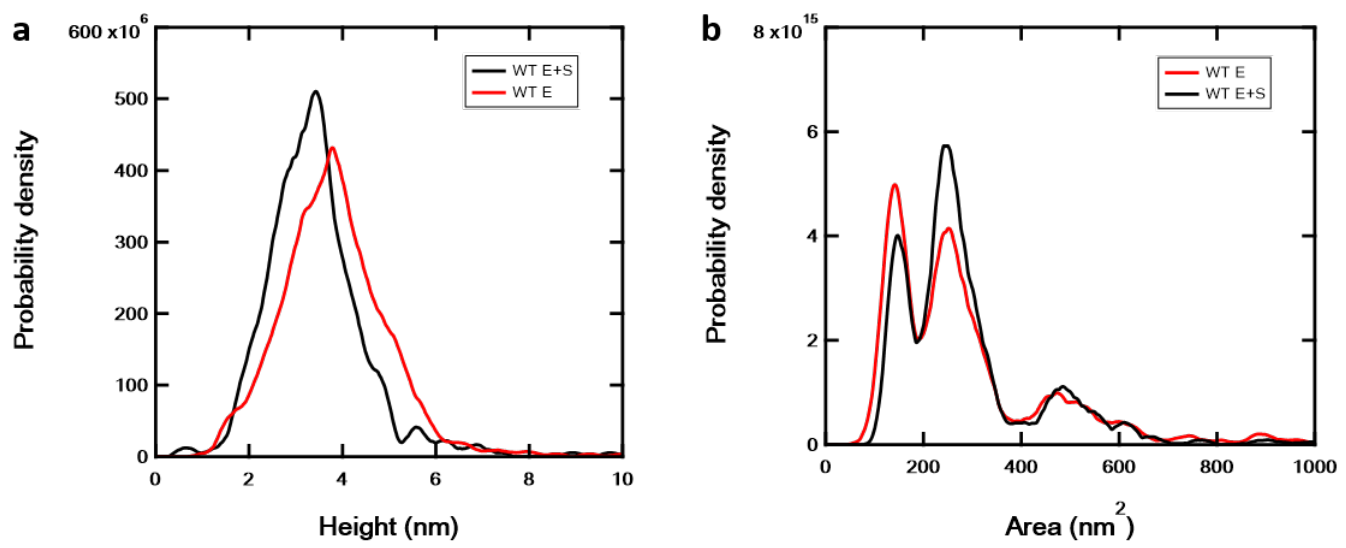

Figure 49: Height and area histograms of the wild type enzyme with (red) and without (black) substrate.
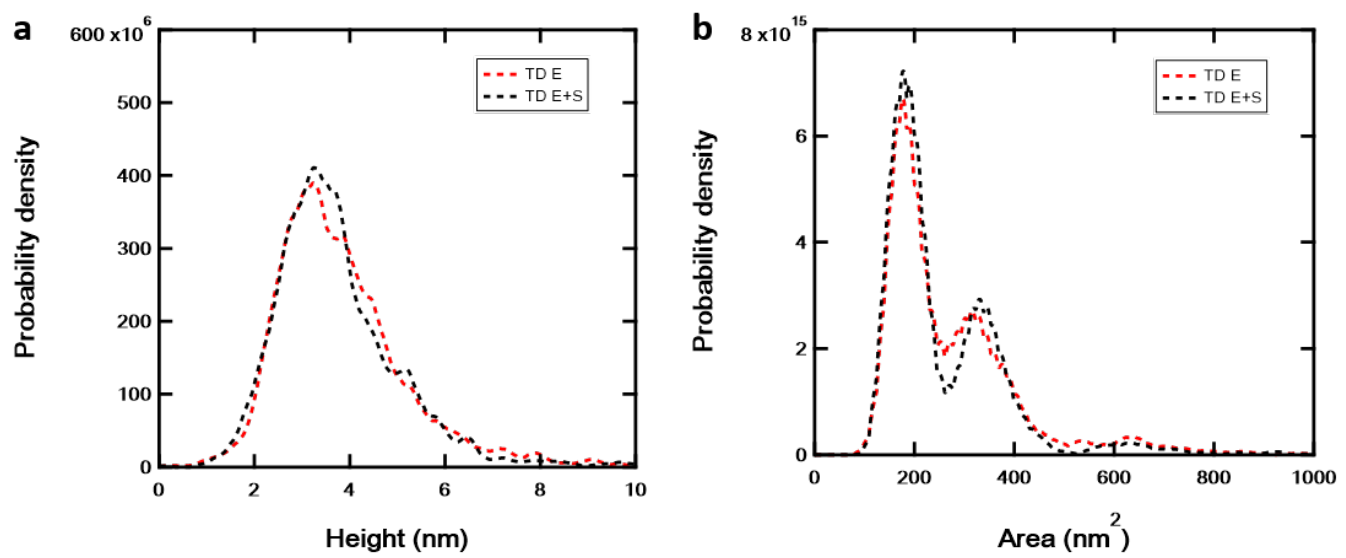

Figure 50: Height and area histograms of tandem dimer enzymes with (red) and without (black) substrate.

Upon inspection of the obtained AFM images, the enzymes on the mica surface appeared to consist of many small smudges (for example, Figure 47 top left panel). After adding substrate, the surface conditions appeared to improve. This improvement also extended to enzymes that were pre-incubated with substrate prior to being 
adsorbed onto mica surface. We evaluated the effects of this pre-incubation step for 15 minutes, 4 hours, and 24 hours to study the effect of substrate to the condition of the AFM measurements. AFM images were acquired (Figure 51) and a comparison was made to the previously collected AFM images. Histograms of height, area, and volume were generated for each condition. Again, the notation used was WT E\& $\mathrm{S}$ and TD E\& S for enzyme pre-incubated with substrate prior adsorption on mica surface.

\section{activate in solution}

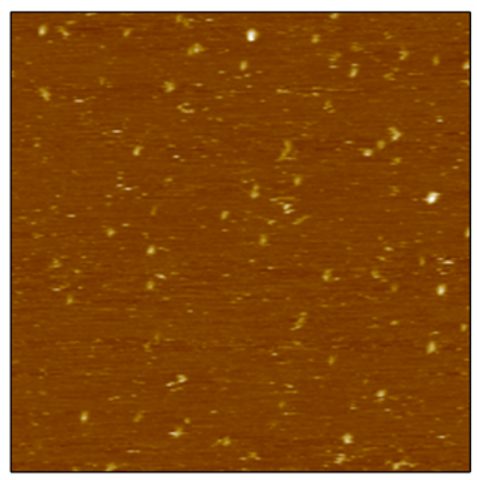

WT E\&S

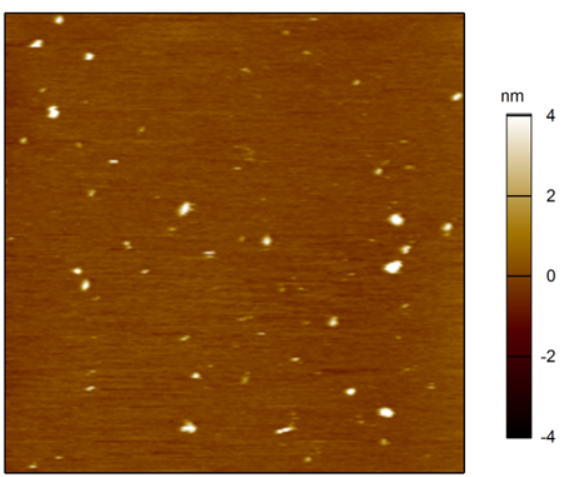

TD E\&S

Figure 51: AFM images of the wildtype (left) and tandem dimer (right) enzymes exposed to substrate in solution for 15 minutes prior to adsorbing onto mica surface.

The AFM images shown in Figure 51 illustrate the improvement of the surface condition in both wildtype and tandem dimer enzymes that comes with pre-incubation with substrate. The wildtype enzymes under this condition also appeared to be smaller in height than those without pre-incubating with the substrate. The height and area histograms for wildtype enzymes incubated with substrate prior to adsorption on the mica surface were generated and shown in Figure 52a and 52b, respectively. The 15-minute incubation was plotted with the blue solid line, the four hour incubation was plotted with the green solid line, and the 24 hour incubation was plotted 
with the pink solid line.
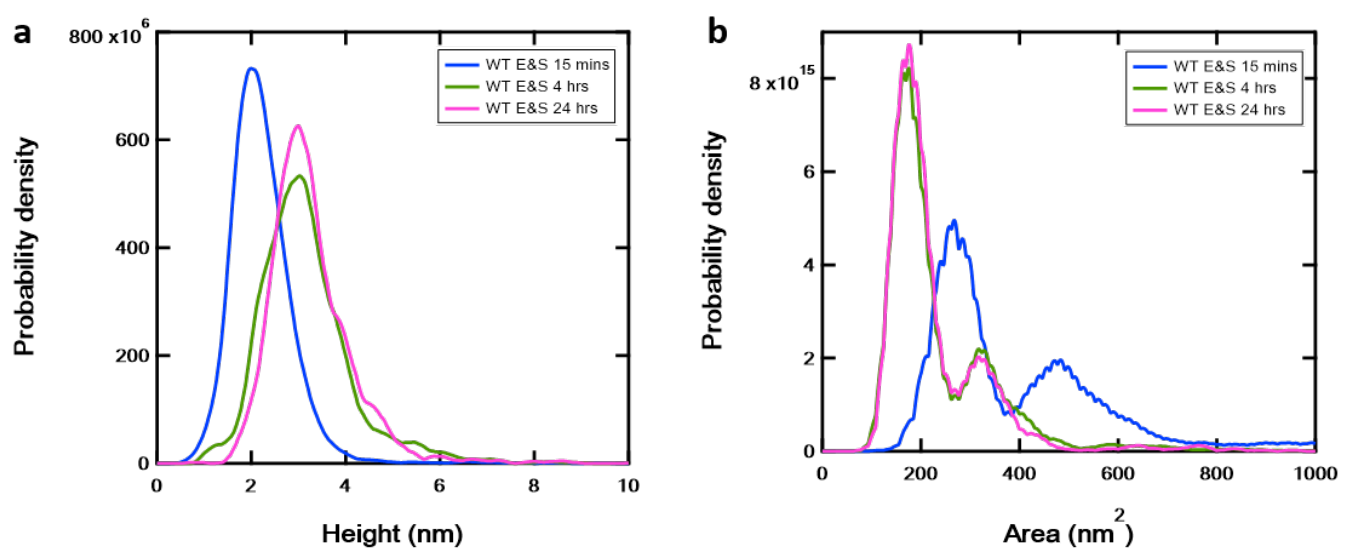

Figure 52: Height and area distribution of the wild type enzyme with different pre-incubation times.

Both height and area histograms indicated no significant difference between 4 and 24 hours of incubation with the substrate prior to adsorption on the mica surface. Under all conditions, the height of the enzymes was reduced after the substrate was introduced. The heights of the enzymes after 4 and 24 hours exposure to the substrate were close to that of the enzyme with substrate added after mica adsorption. The reduction in height for a 15-minute exposure was drastic. In contrast to the height, the area of the enzyme increased after 15 minutes exposure. In order to directly compare all conditions altogether, the histograms of height, area, and volume of each condition are shown in Figure 53a, 53b, and 53c respectively. 

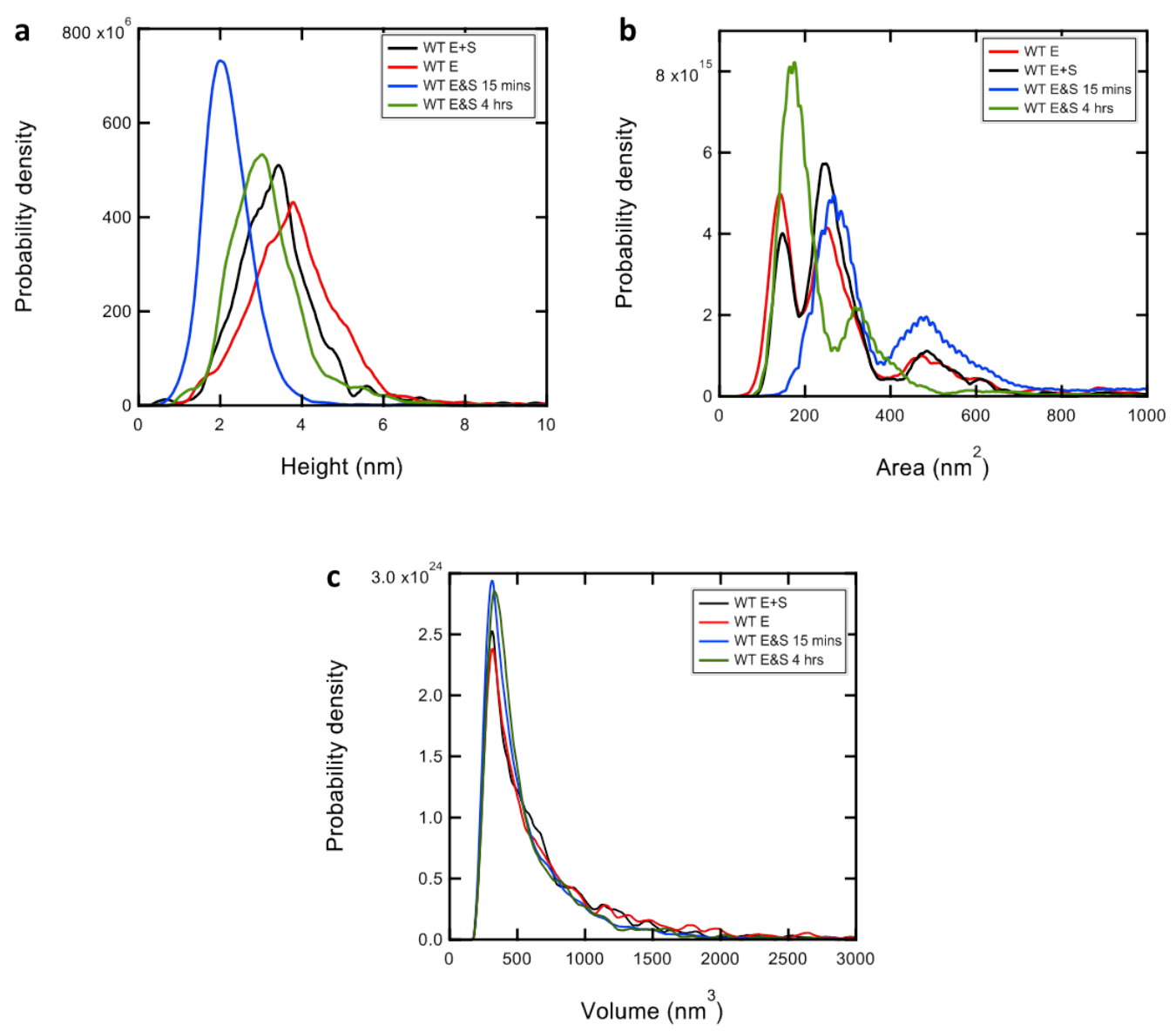

Figure 53: Height, area, and volume distributions of the wild type enzyme with different pre-incubation times. 


\section{Preliminary results and future directions}

This thesis has demonstrated that even highly complicated system such as the general secretory system of $E$. coli can be investigated using single molecule AFM technology. We have used this tool to complement decades of study in the field with bulk biochemical methods. Indeed, AFM illuminated many aspects of the Sec system. Nucleotide dependent conformational changes of SecA were shown by direct observation [24]. The next systematical study of such conformations was to investigate the effect of nucleotides on translocase conformations during the hydrolysis cycle. Proteoliposomes $\mathrm{Y} \cdot \mathrm{A}$ were exposed to nucleotides prior to adsorption on a freshly cleaned glass surface. AFM images were taken in the Apo state, in the presence of $\mathrm{ATP}$, and in the presence of $\mathrm{ADP}-\mathrm{AlF}_{3}(\mathrm{ADPAlF})$, an ATP analog that stabilizes or traps SecA in its transition state $[84,96]$. Representative AFM images of the apo, ATP exposed, ADPAlF exposed conditions are shown in Figure 54a, b, and c, respectively. AFM images were subjected to Hessian blob analysis and histograms were extracted for comparison [79]. Height histograms of translocases in its Apo state (red), exposed to ATP (black), and exposed to ADPAlF (blue) are shown in Figure 55. Area histograms are shown in Figure 56. The number of particles included in the analysis are $\mathrm{N}_{A p o}=1975, \mathrm{~N}_{A T P}=5627, \mathrm{~N}_{A D P A l F}=1410$. 

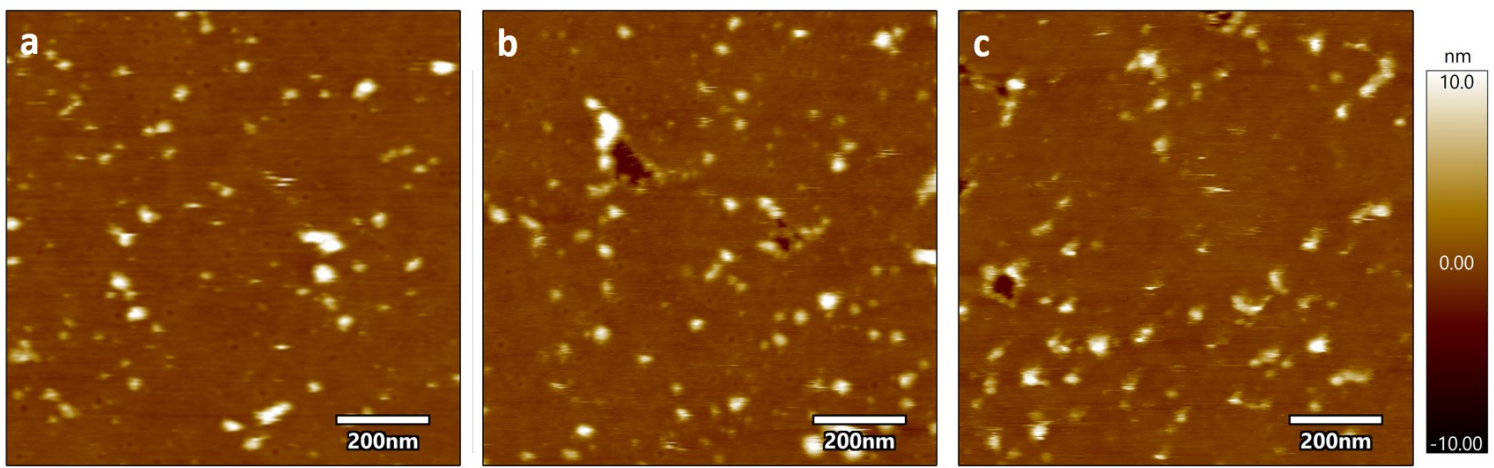

Figure 54: Representative AFM images of translocases on glass surfaces in apo state (a), in the presence of ATP (b), and in the presence of ADPAlF (c). All images are shown with the same color scale for direct comparison.

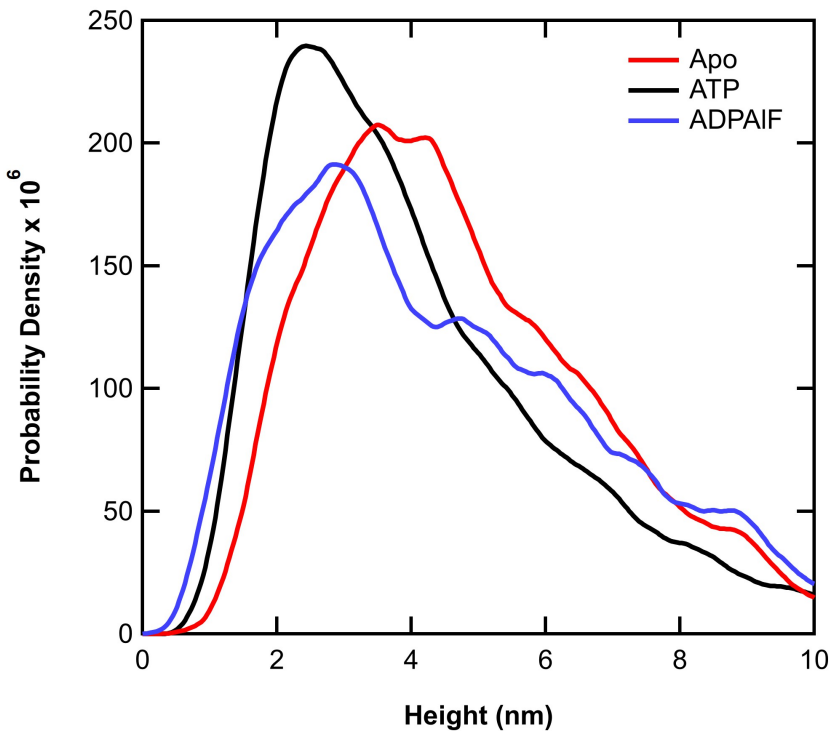

Figure 55: Height histograms of translocases on glass surfaces in apo state (red), exposed to ATP (black), and exposed to ADPAlF (blue). $\mathrm{N}_{A p o}=1975, \mathrm{~N}_{A T P}=$ $5627, \mathrm{~N}_{A D P A l F}=1410$. 


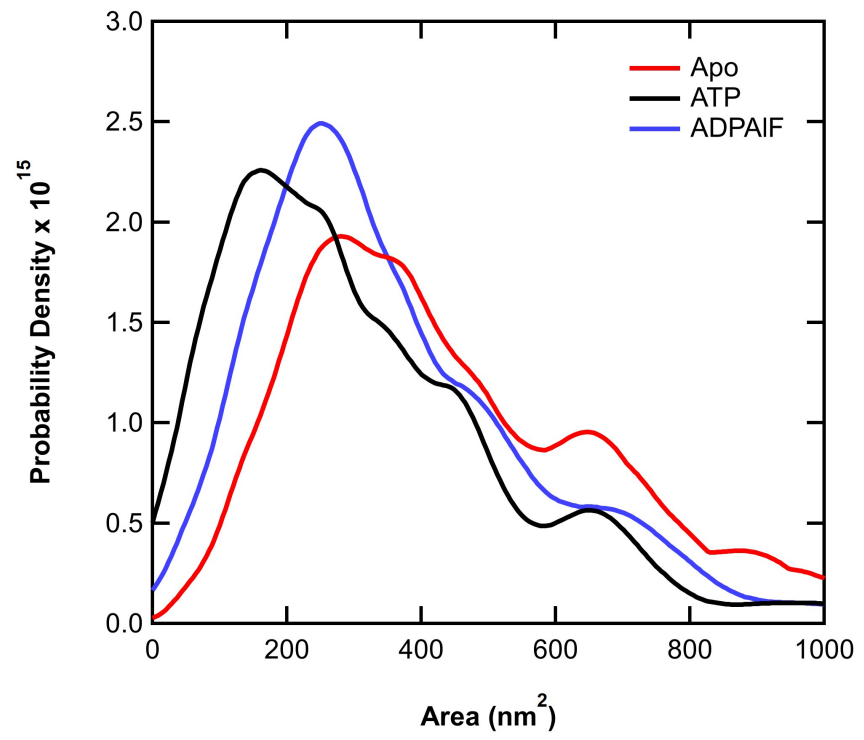

Figure 56: Area histograms of translocases on glass surfaces in its apo state (red), exposed to ATP (black), and exposed to ADPAlF (blue).

To enhance the temporal resolution of the investigation, kymographs were taken in the presence of $3 \mathrm{mM}$ ATP. Representative kymographs obtained from translocases on glass surface are shown in Figure 57. Figure 57(a) shows a magnified view of translocase on the surface, Figure 57(b) shows a kymograph obtained from the particle in Figure 57(a). Figure 57(c) and (d) show another translocase and its representative kymograph. The temporal resolution of these kymographs is $0.14 \mathrm{~s}$ per line.

These kymographs have revealed distinct and interesting conformational dynamics. For example, Figure 57 shows a translocase undergoing prominently vertical conformational changes. In other words, the prominent structural change in this series of line scans over the same macromolecule is normal to the bilayer surface, indicative of a vertically-oriented piston-like motion. In contrast, Figure 57 shows prominently lateral motion. 

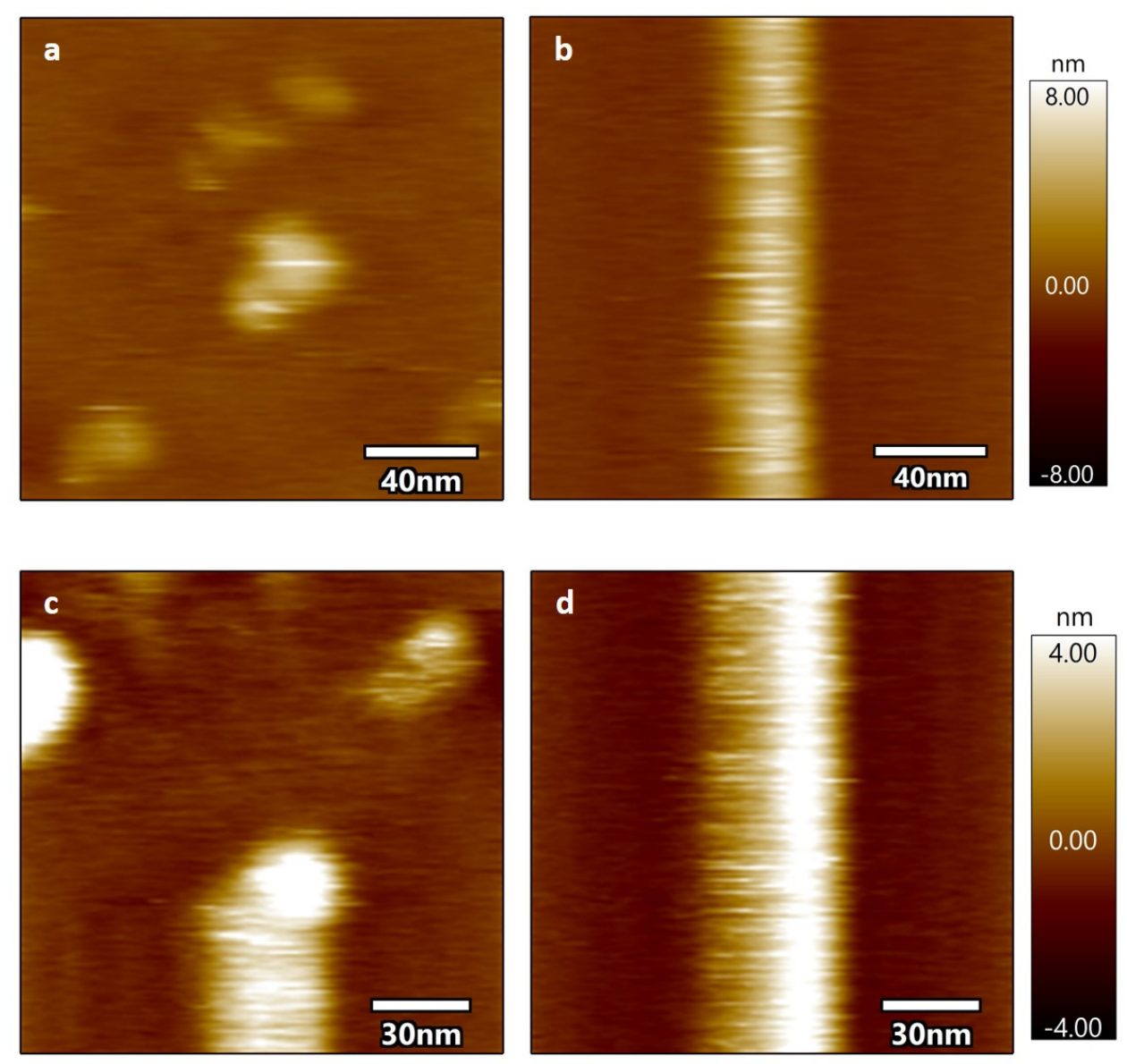

Figure 57: Kymographs were taken in the presence of ATP on the surface. (a) Shows a magnified view of translocase on glass surface. (b) Shows a kymograph obtained from the particle in (a). (c) and (d) show another translocase and its representative kymograph, respectively. The temporal resolution of these kymographs is $0.14 \mathrm{~s}$ per line. Note that the color scale in (a) and (b) is different to that in (c) and (d). The color scale was chosen to highlight the dynamics.

Since the protein binding domain, $\mathrm{PBD}$, of SecA is thought to be the domain that moves the most, it may be responsible for the observed conformational changes. To evaluate this hypothesis and to aid the interpretation of the wild-type data, proteoliposomes $\mathrm{Y} \cdot \mathrm{A} \Delta \mathrm{PBD}$ were adsorbed on glass surfaces. The kymographs of $\mathrm{Y} \cdot \mathrm{A} \Delta \mathrm{PBD}$ were obtained under identical conditions to the wild type. A representative AFM image of $\mathrm{Y} \cdot \mathrm{A} \Delta \mathrm{PBD}$ is shown in Figure 58(a). Kymographs of $\mathrm{Y} \cdot \mathrm{A} \Delta \mathrm{PBD}$ are shown in Figure 58(b) and (c). Figure 58(b) was taken in the apo condition (without ATP). 
Figure 58(c) was taken in the presence of ATP.
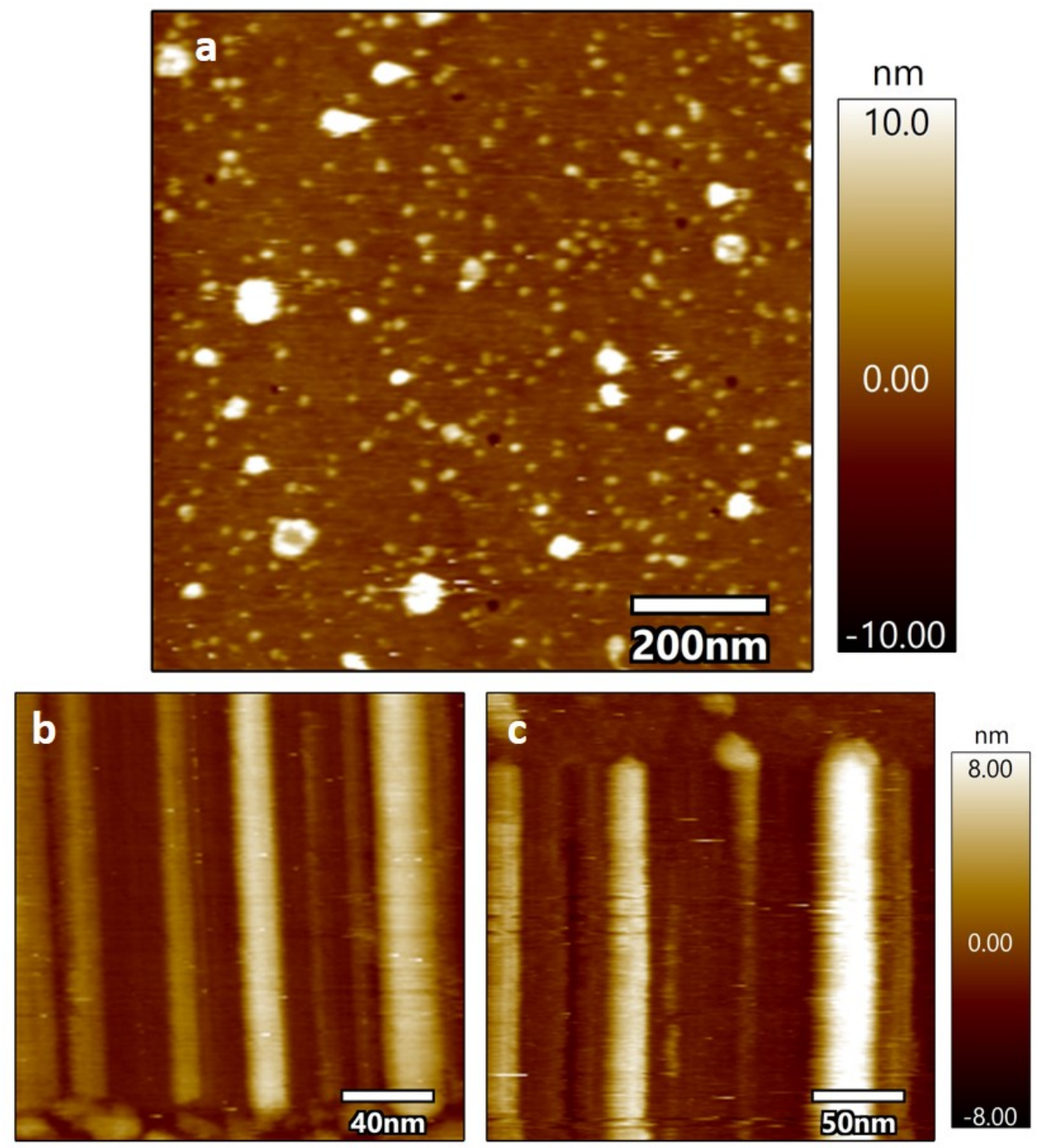

Figure 58: (a) Shows a representative AFM image of $\mathrm{Y} \cdot \mathrm{A} \triangle \mathrm{PBD}$ on glass surface. Kymographs of $\mathrm{Y} \cdot \mathrm{A} \triangle \mathrm{PBD}$ in an apo state are shown in (b). (c) Shows kymographs of $\mathrm{Y} \cdot \mathrm{A} \triangle \mathrm{PBD}$ obtained in the presence of ATP. The temporal resolution of the kymographs is 0.13 s per line.

Next, we attempted to understand the conformational dynamics of translocases on glass surfaces undergoing translocation. We adsorbed proteoliposomes Y·A on freshly cleaned glass, heavily rinsed and imaged the surface with AFM to verify coverage. Then, the reaction mixture containing $1 \mu \mathrm{M} \mathrm{SecB}$, precursor protein $(1 \mu \mathrm{M} \mathrm{pOmpA})$, 
and $3 \mathrm{mM}$ ATP was added onto the surface. The addition of the reaction mixture activated translocation activity. Several AFM images were taken for analysis. Figure 59 reveals translocases on glass surface after $\mathrm{SecB}$, precursor proteins, and ATP were added to the surface.

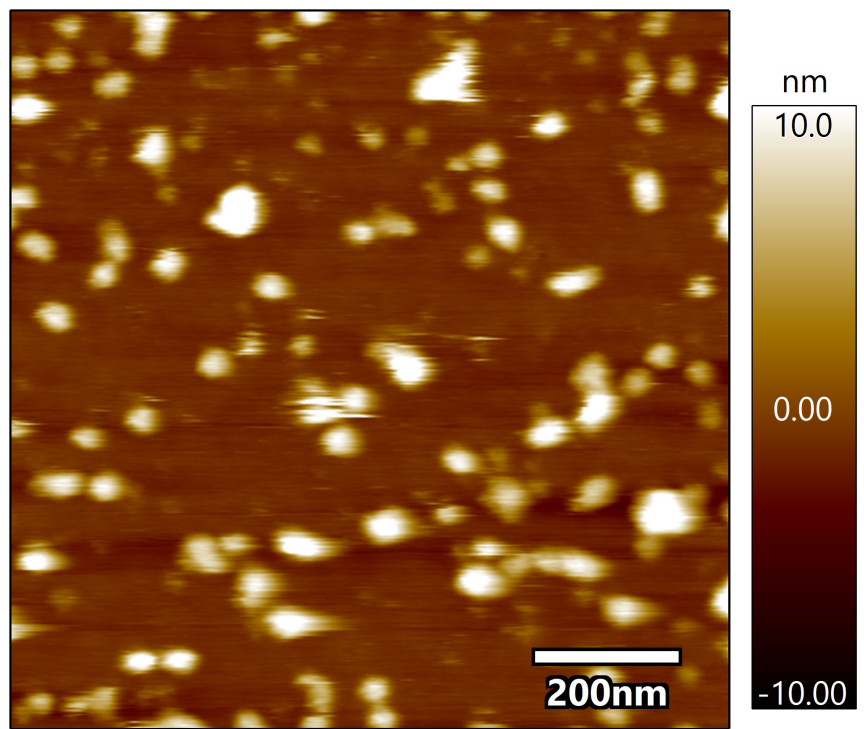

Figure 59: Proteoliposomes Y.A were adsorbed on the surface. Reaction mixture containing SecB, pOmpA, and ATP was added to the surface. This AFM image was taken in the presence of all key components of the active Sec system.

The conformational dynamics of the translocases undergoing translocation can be studied at an enhanced resolution via kymographs. Once the AFM was used to verify that the proper imaging conditions had been achieved, the slow scan axis was disabled and kymographs of several individual translocases were collected. Figure 60 shows representative kymographs obtained from translocases on glass surface undergoing translocation of pOmpA. 

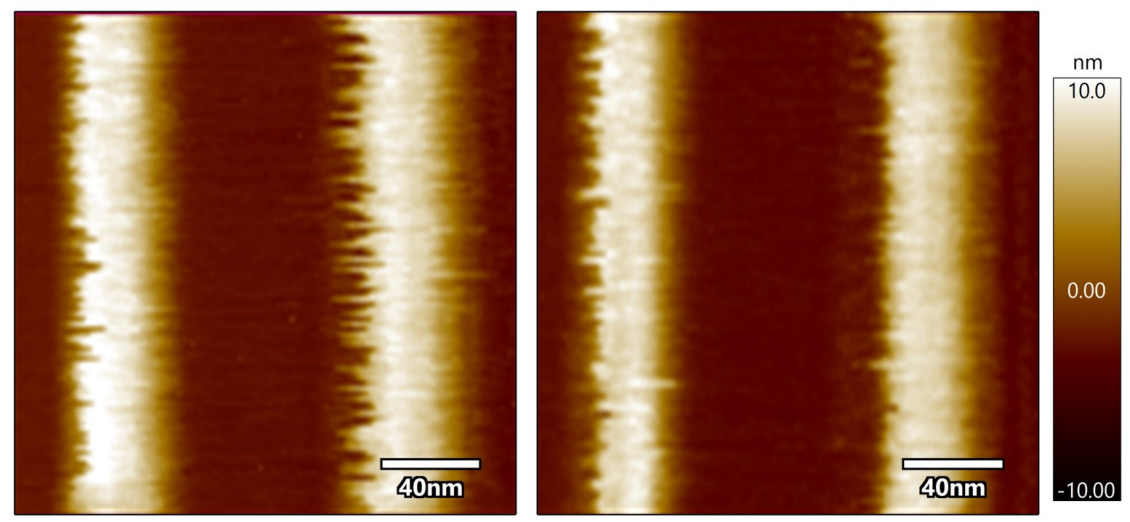

Figure 60: Kymographs of translocases undergoing pOmpA translocation. (a) Shows kymographs of two translocases. (b) Shows kymographs of the same translocase shown in (a) at later time, reflecting the stability of our AFM. The temporal resolution of these two particular kymographs were $0.5 \mathrm{~s}$.

In order to analyze kymographs, we plotted height histograms of the backbone (central location of each line), and both the left and right perimeters $(+/-3$ pixels). This was done to decompose two modes of potential movement of the translocase: the vertical (membrane normal or perpendicular) mode, and the lateral (membrane parallel) direction. We note the perpendicular direction appears as a change in the height of the kymograph. The parallel direction is the motion in the image plane which appears as a change in the width of the kymograph. 

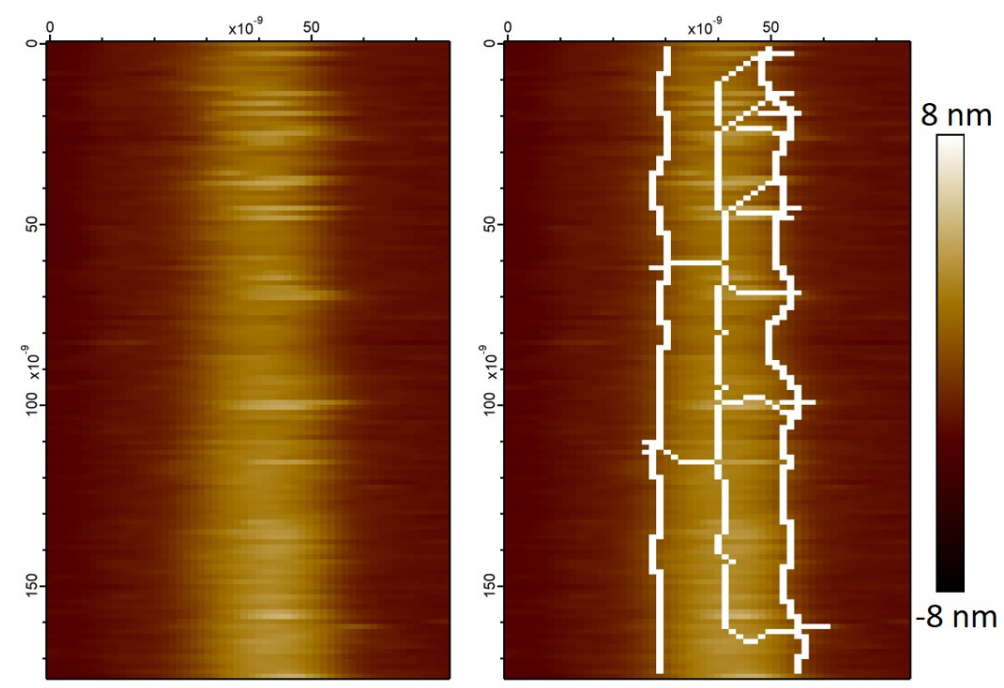

Figure 61: (a) Shows a kymograph of translocase on glass surface in the presence of ATP. (b) Shows kymograph overlaid with the outline of perimeter and the backbone extracted from our kymograph analysis program.
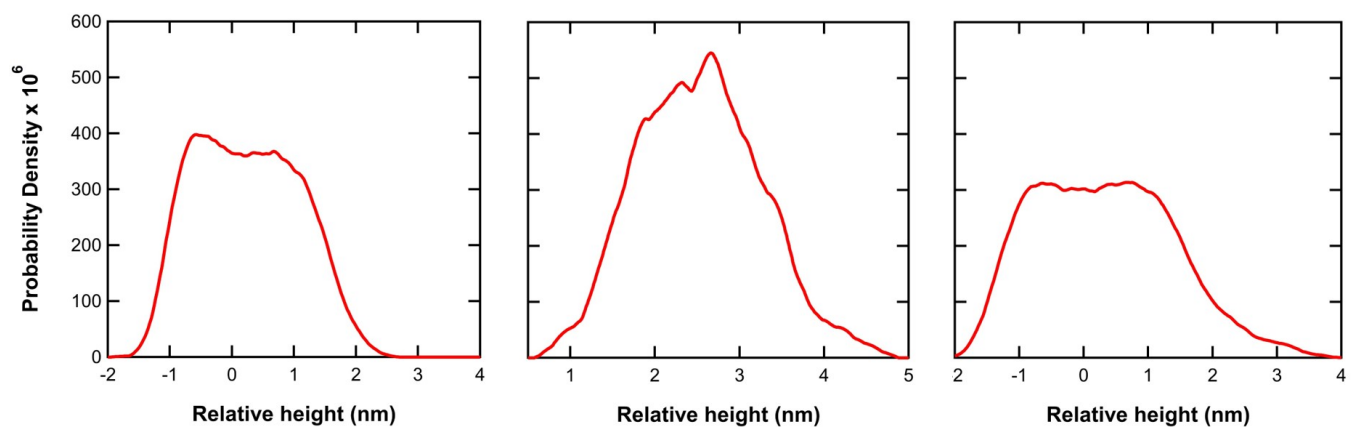

Figure 62: Height histograms extracted from a kymograph obtained from a translocase in the presence of ATP. (a) Shows a height histogram obtained from the left perimeter $+/-3$ pixels. (b) Shows a height histogram obtained from the central pixels. (c) Shows a height histogram obtained from the right perimeter $+/-3$ pixels. Note that all histograms exhibit multiple modes, indicative of distinct conformational states that are transiently occupied.

Our investigation was not only limited to the use of kymographs to probe the dynamics at enhanced temporal resolution, additionally, we also analyzed height distributions of active translocases translocating pOmpA as a function of time. Figure 63 shows height distributions of translocases undergoing pOmpA translocation on 
glass surface. In order to track the dynamics as a function of time with a sufficient number of participants, we extracted height histograms from AFM images taken from different locations and stacked them over one another so that we can visually observe the dynamics. Moreover, we focused on a subset of translocases on the surface by taking AFM images of these populations at one location over a period of time and extracted height histograms. The histograms were shown in Figure 64 . 


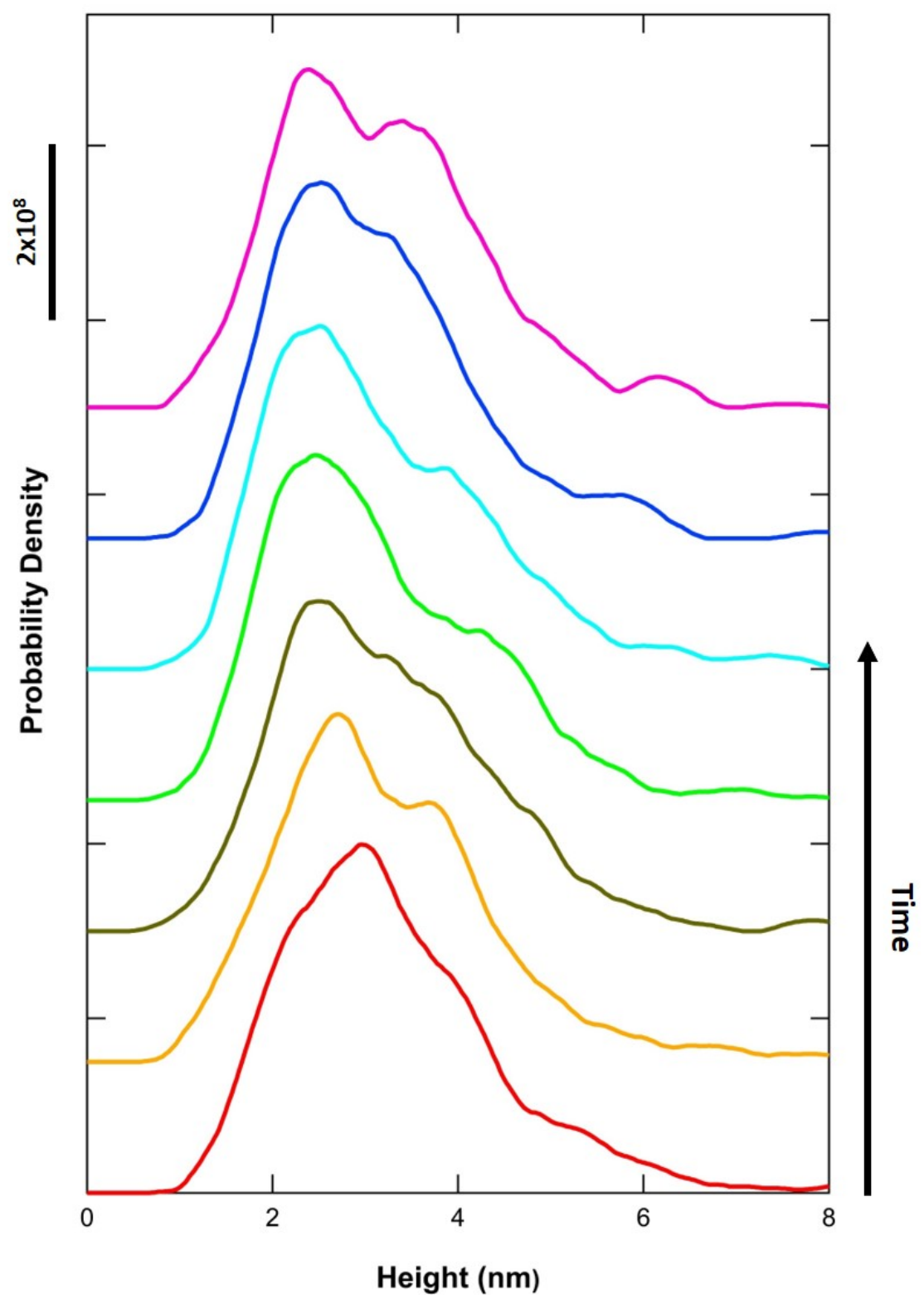

Figure 63: Height distributions of translocases undergoing pOmpA translocation on glass surface from several locations were extracted and stacked over one another to visually present conformational dynamics. 


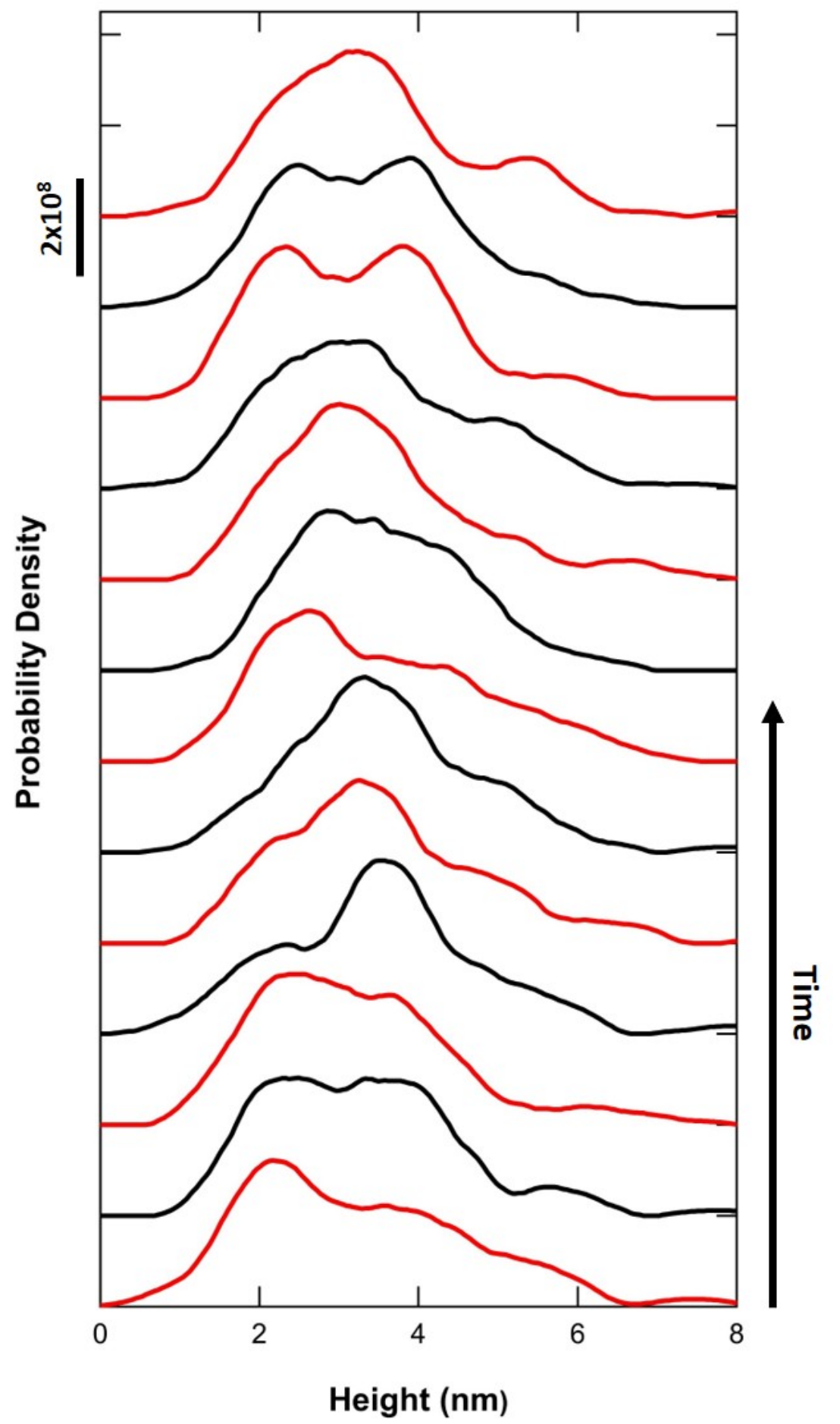

Figure 64: Height distributions of translocases undergoing pOmpA translocation on glass surface from the same location over a period of time were extracted and stacked over one another to visually present conformational dynamics. 
Because translocase complexes can disassemble, we further attempted to deconvolve the fraction of intact translocases (SecYEG-SecA) from translocons, SecYEG, in the absence of SecA. We extracted the fraction of total features of SecYEG alone and SecYEG.SecA using height. From our previous work we know that the cytoplasmic side height of SecYEG above the membrane is typically in the range of 1-3.2 $\mathrm{nm}$, whereas SecYEG-SecA is higher, typically 3.2-8 nm [24,41]. The fraction of SecYEG alone and translocases SecYEG.SecA as a function of time extracted from data shown in Figure 64 is plotted in Figure 65.

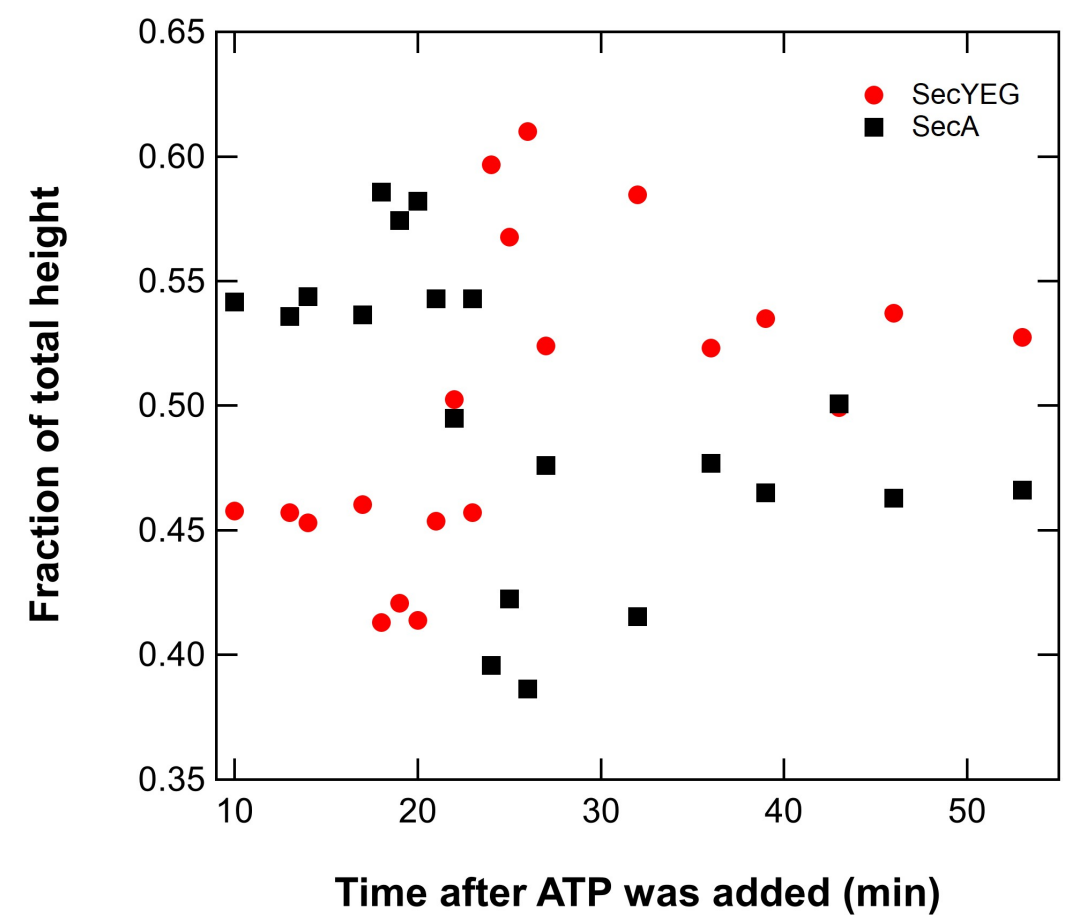

Figure 65: Fraction of translocons alone (SecYEG, red) and intact translocases (SecYEG-SecA, black) as a function of time extracted from data shown in Figure 64. Though the data exhibit significant scatter, there appears to be a trend towards empty translocons (i.e., lacking SecA) over time during the translocation reaction.

Though perliminary, the data in this section suggest that AFM can continue to shed light on fundamental processes involving the translocation of proteins across membranes. In particular, here we show that AFM has the capability of following 
individual translocase complexes during the translocation reaction. With additional control experiments and analyses, this type of data could shed direct light on the mechanism of translocation. It may help resolve the long-standing debate in the field whether the translocase acts as a molecular motor with an ATP dependent power stroke, or more like a Brownian ratchet where ATP binding and hydrolysis plays more of a regulatory role. Note that in vivo, protonmotive force also plays important role in the translocation mechanism. 


\section{Conclusions}

We have used a radiolabeling method to verify ATP hydrolysis activities of SecA on surfaces. The method had sufficient sensitivity to distinguish all known levels of ATP hydrolysis activities associated with the Sec system: basal, translocase activated, and translocation associated. SecA adsorbed on the surface continued to hydrolyze ATP at a comparable rate to those in solution during a time period appropriate for AFM measurements (2 hours). All levels of ATP hydrolysis activities had $>70 \%$ agreement to the traditional in-solution measurements. The free solution containing soluble SecA dissociated from the translocase complexes exposed to the full system maintained a high ATP hydrolysis activity level indicating conformational hysteresis of SecA. ATP hydrolysis activity of surface adsorbed SecA was correlated to the conformations and conformational dynamics of SecA observed in AFM measurements.

Translocation activity of the translocases adsorbed on surfaces were confirmed. Glass provided an environment that promoted more translocation activity than mica. AFM measurements revealed the precursor protein species dependent conformations of translocases engaging in translocation on the surface. Apparent rate constant of translocation activity on glass surface was extracted and compared to that in solution. The on-surface translocation rate was 10-fold lower than that measured in solution. This was likely due to the frictional drag force introduced by the underlying surface.

Chemomechanical coupling was calculated. The number obtained from glass surface was compared to that from mica surface, the in-solution measurement, and the traditional reconstitution system where SecA was added to the proteoliposome SecYEG after the reconstitution. Translocases on glass had 2-fold reduction in chemomechanical coupling. When comparing the chemomechanical coupling calculated from translocases on mica to that calculated from glass, the system on mica had a much 
higher value. This was likely due to the inherited surface roughness of glass which could provide extra spaces for precursor to occupy after being translocated.

NR results provided additional information on the submembrane space which was inaccessible to AFM. The submembrane space obtained from NR was $9 \AA$. Although small, this submembrane space was sufficient to accommodate translocated precursor protein. Thus, NR can be used to complement AFM measurements.

In summation, high precision single molecule methods such as AFM are poised to reveal the intricate mechanisms underlying the Sec system activity. 


\section{Appendix:}

\section{A On-surface activity assays}

\section{A.1 On-surface basal ATP hydrolysis activity}

Caution: All assays using ${ }^{32} \mathbf{P}$ must be done using appropriate shielding. Additionally, when moving with ${ }^{32} \mathrm{P}$, it must also be shielded.

1. Deposit $50 \mu \mathrm{L}$ of $2 \mu \mathrm{M}$ SecA in $10 \mathrm{mM}$ HEPES, pH 7.6, $100 \mathrm{mM} \mathrm{KAc}, 5$ $\mathrm{mM} \mathrm{MgAc} 2$ onto freshly cleaved mica surface on Teflon lined metal disc for 15 minutes at room temperature.

2. Rinse the surface 10 times with the same buffer.

3. Add $50 \mu \mathrm{L}$ of $3 \mathrm{mM}\left[\gamma^{-32} \mathrm{P}\right]$ ATP $(0.908 \mathrm{nCi} / \mu \mathrm{L}), 10 \mathrm{mM}$ HEPES, $\mathrm{pH}$ 7.6, 100 $\mathrm{mM} \mathrm{KAc,} 5 \mathrm{mM} \mathrm{MgAc} 2$ to the surface.

Note: Make sure that all the radioactive wastes are properly disposed in a clearly labeled waste container and separated from regular wastes.

4. At corresponding times, pipet $4 \mu \mathrm{L}$ of the solution off the surface and mix with $4 \mu \mathrm{L}$ of $50 \mathrm{mM}$ EDTA to stop the ATP hydrolysis reaction. This mixture is called the reaction stop mixture.

Note: EDTA is a chelating agent that effectively binds with $\mathrm{Mg}^{2+}$ ion. SecA requires $\mathrm{Mg}^{2+}$ to function in hydrolyzing $\mathrm{ATP}$. Thus, the removal of $\mathrm{Mg}^{2+}$ inhibits SecA ATP hydrolysis activities.

5. Spot $2 \mu \mathrm{L}$ of the reaction stop mixture onto the thin layer chromatography (TLC) plate and let the spots dry.

Note: Measure and mark the 20 x $20 \mathrm{~cm}$ TLC plate before spotting the solution. 
Leave a $1.5 \mathrm{~cm}$ space from the left and right edges of the plate, and $2.5 \mathrm{~cm}$ from the bottom edge of the plate. One plate fits up to 15 spots.

6. Place the dried TLC plate in the $125 \mathrm{mM} \mathrm{KH}_{2} \mathrm{PO}_{4}$ solution in the closed chromatography tank behind a shield for the radioactivity of ${ }^{32} \mathrm{P}$.

Note: The $\mathrm{KH}_{2} \mathrm{PO}_{4}$ solution level should be just below the sample line.

7. Let the fluid rise to $2.5 \mathrm{~cm}$ below the top edge of the TLC plate, to ensure that the chromatography is completed.

8. Dry the plate in an oven at $70{ }^{\circ} \mathrm{C}$.

9. Wrap the plate in plastic wrap to prevent potential contamination of the imaging plate from radioactive particles.

10. Expose the wrapped plate to the PhosphorImager in a closed cassette for 15 minutes.

Note: The exposure time can be adjusted to accommodate the radioactivity of ${ }^{32} \mathrm{P}$.

11. Scan the PhosphorImager with a scanner such as the Amersham Typhoon.

12. Quantify the radioimage using software such as ImageQuant.

13. During quantification, make sure that the background was subtracted properly and the entire areas were included. Thus, the percent ATP hydrolyzed is appropriately quantified.

14. Convert \% ATP hydrolyzed to mol ATP hydrolyzed and normalize with mol SecA available on the surface assuming complete coverage of the surface.

Note: Multiply \%ATP with the concentration and volume of the ATP used in 
the experiment to get mol ATP. Use the SecA crystallographic dimensions (4 nm $\mathrm{x} 8 \mathrm{~nm}$ ) and the mica dimensions (10 $\mathrm{mm}$ diameter circle) to estimate number of mol of SecA on the surface. SecA was estimated to be 3 pmol on the surface.

\section{A.2 On-surface translocase activated ATP hydrolysis activ- ity}

1. Deposit $50 \mu \mathrm{L}$ of $80 \mathrm{nM}$ proteoliposomes SecYEG co-assembled with SecA (Y. A) in buffer containing $10 \mathrm{mM}$ HEPES, pH 7.6, $300 \mathrm{mM} \mathrm{KAc}, 5 \mathrm{mM} \mathrm{MgAc} 2$ on to a freshly cleaved mica surface on a Teflon lined metal disc.

2. Place the surface in a closed, water saturated environment (petri dish) on ice.

3. Let the surface incubate on ice for 1 hour to ensure a complete coverage of the lipid bilayer on the surface.

4. Rinse the surface with $50 \mu \mathrm{L}$ of the same buffer 10 times.

5. Add $50 \mu \mathrm{L}$ of $3 \mathrm{mM}\left[\gamma^{-32} \mathrm{P}\right]$ ATP $(0.908 \mathrm{nCi} / \mu \mathrm{L}), 10 \mathrm{mM}$ HEPES, pH 7.6, 300 $\mathrm{mM} \mathrm{KAc,} 5 \mathrm{mM} \mathrm{MgAc} c_{2}$ to the surface.

6. At corresponding times, pipet $4 \mu \mathrm{L}$ of the solution off the surface and mix with $4 \mu \mathrm{L}$ of $50 \mathrm{mM}$ EDTA to stop the ATP hydrolysis reaction.

7. Spot $2 \mu \mathrm{L}$ of the reaction stop mixture onto the thin layer chromatography (TLC) plate and let the spot dry.

8. Place the dried TLC plate in the $125 \mathrm{mM} \mathrm{KH}_{2} \mathrm{PO}_{4}$ solution in the closed chromatography tank behind a shield for of ${ }^{32} \mathrm{P}$.

9. Let the fluid rise to $2.5 \mathrm{~cm}$ below the top edge of the TLC plate, to ensure that the chromatography is completed. 
10. Dry the plate in the oven at $70^{\circ} \mathrm{C}$.

11. Wrap the plate in plastic wrap to prevent potential contamination from radioactive particles.

12. Expose the wrapped plate to the PhosphorImager in a closed cassette for 15 minutes.

13. Scan the PhosphorImager with Amersham Typhoon

14. Quantify the radiograph using software such as ImageQuant

15. During quantification, make sure that the background was subtracted properly and the whole spot sizes were included in the analysis lane. Thus, percent ATP hydrolyzed is quantified correctly.

16. Convert percent ATP hydrolyzed to mol ATP hydrolyzed and normalize with mol SecA available on the surface assuming complete coverage of the surface. Note: Multiply \%ATP with $2 \mu \mathrm{L}$ x $3 \mathrm{mM}$ to get mol ATP. Use AFM images to estimate number of mol of SecA available on the surface. We estimated 0.03 pmol of translocase complexes available on mica surface.

\section{A.3 On-surface translocation associated ATP hydrolysis ac- tivity}

1. Deposit $50 \mu \mathrm{L}$ of $80 \mathrm{nM}$ of proteoliposomes containing SecYEG co-assembled with SecA (Y·A) in buffer containing 10 mM HEPES, pH 7.6, 300 mM KAc, 5 $\mathrm{mM} \mathrm{MgAc} c_{2}$ on to a freshly cleaved mica surface on a Teflon lined metal disc.

2. Place the surface in a closed environment (petri dish) on ice. 
3. Let the surface incubate on ice for 1 hour to ensure a complete coverage of the membrane on the surface.

4. Rinse the surface with $50 \mu \mathrm{L}$ of the same buffer 10 times.

5. Add $50 \mu \mathrm{L}$ of $3 \mathrm{mM}\left[\gamma_{-}{ }^{32} \mathrm{P}\right]$ ATP $(0.908 \mathrm{nCi} / \mu \mathrm{L}), 1 \mu \mathrm{M}$ SecB, $1 \mu \mathrm{M}$ precursor protein (pOmpA or pGBP), 10 mM HEPES, pH 7.6, 300 mM KAc, 5 mM $\mathrm{MgAc}_{2}, 2 \mathrm{mM}$ DTT, $1 \mathrm{mM}$ EGTA to the surface

Note: EGTA is also a chelating agent that has a higher affinity for $\mathrm{Ca}^{2+}$ than its affinity for $\mathrm{Mg}^{2+}$. Thus, it binds and gets rid of trace amounts of $\mathrm{Ca}^{2+}$ in the solution. The presence of $\mathrm{Ca}^{2+}$ leads to precursor protein folding prematurely. Note: Concentration of $\mathrm{Mg}^{2+}$ must be higher than that of EGTA so that there is sufficient $\mathrm{Mg}^{2+}$ for the ATPase to function.

6. At corresponding times, pipet $4 \mu \mathrm{L}$ of the solution off the surface and mix with $4 \mu \mathrm{L}$ of $50 \mathrm{mM}$ EDTA to stop the ATP hydrolysis reaction and follow the same procedure described earlier in basal and translocase activated ATP hydrolysis procedure.

\section{A.4 On-surface translocation activity}

1. Deposit $50 \mu \mathrm{L}$ proteoliposomes of $80 \mathrm{nM}$ SecYEG coassembled with $\operatorname{Sec} \mathrm{A}(\mathrm{Y} \cdot \mathrm{A})$ in buffer containing $10 \mathrm{mM}$ HEPES, pH 7.6, $300 \mathrm{mM} \mathrm{KAc}, 5 \mathrm{mM} \mathrm{MgAc}$ on to a freshly cleaned glass surface (KOH followed by oxygen plasma. The treatment procedure is discussed in A.5) or a freshly cleaved mica on a Teflon lined glass slide.

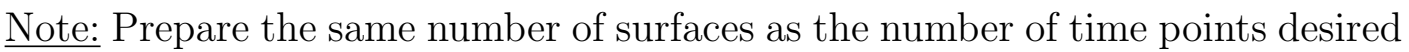
to acquire the translocation activity graph. 
2. Place the surface in a closed, water-saturated environment (petri dish).

3. Let the surface incubate on ice for 1 hour to ensure coverage of the membrane over a large area.

4. Rinse the surface with $50 \mu \mathrm{L}$ of the same buffer 10 times.

5. Add $50 \mu \mathrm{L}$ of $3 \mathrm{mM}$ ATP, $1 \mu \mathrm{M}$ SecB, $1 \mu \mathrm{M}$ radioactive precursor protein $\left({ }^{14} \mathrm{C}\right.$ pOmpA or ${ }^{14} \mathrm{C}$-pGBP), $10 \mathrm{mM}$ HEPES, pH 7.6, $300 \mathrm{mM} \mathrm{KAc,} 5 \mathrm{mM} \mathrm{MgAc} 2$, $2 \mathrm{mM}$ DTT, $1 \mathrm{mM}$ EGTA to the surface.

Note: Always add SecB to the solution mix before precursor protein to prevent the precursor protein from folding prematurely, which would ruin the experiment. Use a pipette tip to thoroughly mix the solution on the surface.

6. Keep the surface in a water saturated environment at room temperature.

7. At predetermined times, add $150 \mu \mathrm{L}$ of $6 \mathrm{M}$ urea to the surface. Use a pipette tip to thoroughly mix the urea solution with the surface solution.

Note: The urea solution must be freshly made on the day of experiment.

8. Vigorously rinse the surface by depressing and releasing the pipette several times. Be careful not to spill the fluid off the surface.

9. Rinse the surface with $50 \mu \mathrm{L}$ of $10 \mathrm{mM}$ HEPES, pH 7.6, $300 \mathrm{mM} \mathrm{KAc}, 5 \mathrm{mM}$ $\mathrm{MgAc}_{2}, 2 \mathrm{mM}$ DTT, 1 mM EGTA buffer 10 times.

10. Add $70 \mu \mathrm{L}$ of proteinase $\mathrm{K}$ solution at 19 units $/ \mathrm{mL}$ on the surface.

11. Place the surface on ice for 15 minutes.

12. Rinse the surface with the same buffer 10 more times.

13. Pipet $95 \%$ of the remaining surface solution off the surface. 
14. Dry the surface in the oven at $70^{\circ} \mathrm{C}$ for 20 minutes.

15. Wrap the surface with clear plastic wrap.

16. Expose the surface and the standard made from spotting known quantities of the radioactive protein on the surface to the imaging plate in a closed cassette for 48 hours.

17. Develop a radiograph of the surface using a scanner such as GE healthcare Amersham Typhoon.

18. Quantify the translocation activity using quantitative software such as ImageQuant.

19. Create a standard curve from the standard samples and convert intensity of the surface to mol precursor protected on the surface.

20. Perform control experiments in an identical manner described above.

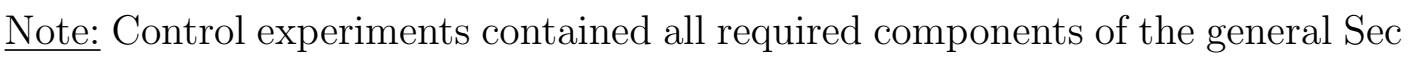
system except SecYEG; SecA; or both SecYEG and SecA as shown in Figure 26

\section{A.5 Preparation of $\mathrm{KOH}$ treated glass cover slips}

1. Cut a $\mathrm{KOH}$ cleaned cover glass slip into four equal pieces. The cleaning procedure using $\mathrm{KOH}$ solution was discussed in reference [8].

2. Rinse with ethanol and DI water in an alternating manner three times to clean the residue.

3. Blow dry with ultra-high purity nitrogen gas. 
4. Plasma clean right before use.

5. Glue the freshly plasma cleaned glass onto the Teflon lined metal disc using 5 -min epoxy.

\section{B In-solution activity assays}

\section{B.1 In-Solution ATP hydrolysis}

Basal ATP hydrolysis

1. Prepare solution containing $1 \mu \mathrm{M}$ SecA in $10 \mathrm{mM}$ HEPES, pH 7.6, $300 \mathrm{mM}$ $\mathrm{KAc}, 5 \mathrm{mM} \mathrm{MgAc} 2$

2. Add ATP to achieve final concentration of $3 \mathrm{mM}\left[\gamma^{-32} \mathrm{P}\right] \operatorname{ATP}(0.908 \mathrm{nCi} / \mu \mathrm{L})$

3. At determined times pipet $4 \mu \mathrm{L}$ of the solution off the surface and mix with 4 $\mu \mathrm{L}$ of $50 \mathrm{mM}$ EDTA to stop the ATP hydrolysis reaction.

4. Spot $2 \mu \mathrm{L}$ of the reaction stop mixture onto the thin layer chromatography (TLC) plate.

5. Place the dried TLC plate in the $125 \mathrm{mM} \mathrm{KH}_{2} \mathrm{PO}_{4}$ solution in the closed chromatography tank behind a shield for radioactivity of ${ }^{32} \mathrm{P}$ such that the solution level is just below the spotted locations.

6. Let the fluid rise to $2.5 \mathrm{~cm}$ below the top edge of the TLC plate, to ensure that the chromatography is completed.

7. Dry the plate in the oven at $70{ }^{\circ} \mathrm{C}$.

8. Wrap the plate in plastic wrap to prevent potential contamination from radioactive particles. 
9. Expose the wrapped plate to the PhosphorImager in a closed cassette for 15 minutes.

10. Scan the PhosphorImager with Amersham Typhoon

11. Quantify the radiograph using software such as ImageQuant

12. During quantification, make sure that the background was subtracted properly and the whole spot sizes were included in the analysis lane. Thus, percent ATP hydrolyzed is quantified correctly.

13. Convert percent ATP hydrolyzed to mol ATP hydrolyzed and normalize with mol SecA available in solution according to the know concentration of ATP and SecA in the reaction solution.

\section{Translocase activated ATP hydrolysis}

1. Prepare solution containing proteoliposome Y·A with $1 \mu \mathrm{M}$ SecYEG concentration in $10 \mathrm{mM}$ HEPES, $\mathrm{pH}$ 7.6, $300 \mathrm{mM} \mathrm{KAc,} 5 \mathrm{mM} \mathrm{MgAc}$.

2. Add ATP to achieve final concentration of $3 \mathrm{mM}\left[\gamma_{-}{ }^{32} \mathrm{P}\right]$ ATP $(0.908 \mathrm{nCi} / \mu \mathrm{L})$.

3. Follow the steps described earlier in basal ATP hydrolysis assay.

\section{Translocation associated ATP hydrolysis}

1. Prepare solution containing proteoliposome Y·A with $1 \mu \mathrm{M}$ SecYEG concentration in10 mM HEPES, pH 7.6, $300 \mathrm{mM} \mathrm{KAc,} 5 \mathrm{mM} \mathrm{MgAc} 2,2 \mathrm{mM}$ DTT, 1 mM EGTA.

2. Add $1 \mu \mathrm{M} \mathrm{SecB}, 1 \mu \mathrm{M}$ precursor protein (pOmpA or pGBP), and $3 \mathrm{mM}\left[\gamma^{32} \mathrm{P}\right]$ $\operatorname{ATP}(0.908 \mathrm{nCi} / \mu \mathrm{L})$.

3. Follow the steps described earlier in basal and translocase activated ATP hydrolysis assays. 


\section{B.2 In-Solution translocation activity}

1. Prepare solution containing final concentration of proteoliposome $\mathrm{Y} \cdot \mathrm{A}$ with 1 $\mu \mathrm{M}$ SecYEG, $1 \mu \mathrm{M}$ SecB, $1 \mu \mathrm{M}$ radioactive precursor protein $\left({ }^{14} \mathrm{C}-\mathrm{pOmpA}\right.$ or ${ }^{14} \mathrm{C}$-pGBP), $3.3 \mathrm{mM}$ ATP in $10 \mathrm{mM}$ HEPES, pH 7.6, $300 \mathrm{mM} \mathrm{KAc,} 5 \mathrm{mM}$ $\mathrm{MgAc}_{2}, 2 \mathrm{mM}$ DTT, 1 mM EGTA

2. At a predetermined time, sample the solution mixture in step 1 and mix with reaction stop containing proteinase $\mathrm{K}$ at 19 units/mL.

3. The solution is subjected to electrophoresis.

4. The gel was stained, rinsed thoroughly, dried and exposed in phosphorimager in a closed cassette for 48 hours.

5. The image was scanned using a scanner such as GE healthcare Amersham Typhoon.

6. The radioactivity of the protected precursor protein was quantified using software such as ImageQuant.

7. The control experiments were done under identical conditions except in the absence of ATP.

\section{Neutron Reflectometry}

1. The NR sample was prepared by chemical vapor deposition of a nominally 100 $\AA$ thick dry thermal $\mathrm{SiO}_{x}$ film on a $75 \mathrm{~mm}$ diameter, $5 \mathrm{~mm}$ thick 100 single crystal silicon wafer $\mathrm{N}$-doped with phosphorus to $1-100 \Omega \cdot \mathrm{cm}$. The deposition was performed by exposing the wafer to 14 min of oxygen flow at $15 \mathrm{~L}$ per min at $700{ }^{\circ} \mathrm{C}$, using a SandVik model 1314 furnace at the NIST Center for 
Nanoscale Science and Technology.

Note: All samples were made and supplied from NCNR.

2. The sample wafer surface was cleaned by sonicating for 2 minutes in a saturated ethanolic solution of potassium hydroxide, followed by sonication-assisted rinses with copious $18 \mathrm{M} \Omega \cdot \mathrm{cm}$ reverse osmosis water.

Note: At the time of experiment, we did not have access to plasma cleaning at NCNR. Thus, the plasma cleaning process was omitted.

3. Y·A proteoliposomes were added at $1 \mu \mathrm{M}$ concentration via a pipette to the pristine $\mathrm{SiO}_{x}$ surface, and droplet spreading was confirmed visually.

4. While still wet, the wafer was mounted in a flow cell so that the $\mathrm{SiO}_{x}$ interface was in contact with a nominally $100 \mu \mathrm{m}$ thick reservoir of buffer.

5. For reflectivity measurements, a monochromatic beam of wavelength $5 \AA$ impinged on the interface between the sample wafer surface and liquid in the 100 $\mu \mathrm{m}$ thick liquid reservoir.

6. The presample collimating slits were chosen to maintain a constant $50 \mathrm{~mm} \times$ $25 \mathrm{~mm}$ illuminated interfacial area for each measured angle $\theta$.

7. The postsample collimation was chosen to allow the entire reflected beam to impinge on the detector, which was positioned at an angle $2 \theta$ relative to the incoming beam direction to measure specular reflection.

8. Each reflectivity curve covered a range in scattering vector $\mathrm{Q}_{z}=4 \pi \lambda^{-1}$ from 0.010 to $0.250 \AA^{-1}$ with a step size of $0.0024 \AA^{-1}$.

9. The reflectivity was calculated as $R=\frac{I\left(Q_{z}\right)-I_{B}\left(Q_{z}\right)}{\left(I_{0}\left(Q_{z}\right)\right.}$, where $\mathrm{I}\left(\mathrm{Q}_{z}\right)$ is the measured count rate (normalized to a much larger monitor count rate to account for 
fluctuations in beam intensity). $\mathrm{I}_{B}\left(\mathrm{Q}_{z}\right)$ is the background intensity, which arises primarily from incoherent scattering from the liquid reservoir and is calculated by linear interpretation of the intensities measured with the detector positioned at $1.5 \theta$ and $2.5 \theta . \mathrm{I}_{0}\left(\mathrm{Q}_{z}\right)$ is the incident beam intensity (also normalized to the monitor count rate) and is directly measured through the silicon substrate at $\theta=0$ with the detector positioned in line with the incident beam.

10. NR data were analyzed using the composition space modeling procedures described previously [74]. Simultaneous optimization of the composition space model for the four buffer conditions was performed on the Bridges $[97,98]$ highperformance computing system using the DREAM Markov Chain Monte Carlo algorithm [99] implemented in the software package Refl1D [100]. CI on parameters and model predictions were calculated from parameter distributions derived from 4.1 million DREAM samples after the optimizer had reached steady state.

\section{Biochemical Methods}

\section{D.1 Purification of Sec Components}

SecYEG was purified from a strain C43 (DE3) suitable for overexpression of membrane protein [101] harboring a plasmid encoding SecE with a His-tag at the N terminus, SecYC329S, C385S, and SecG $[46,102]$. Cells were broken by passage

through a French Pressure Cell $\left(5.5 \times 10^{7} \mathrm{~Pa}\right)$, and the membranes were isolated by centrifugation and solubilized in dodecyl- $\beta$-maltoside (DBM). SecYEG was purified by chromatography using a HisTrap column (GE Healthcare) and stored at -80 ${ }^{\circ} \mathrm{C}$ in $20 \mathrm{mM}$ Tris-Cl (where $1 \mathrm{M}=1 \mathrm{~mol} / \mathrm{L}$ ), $\mathrm{pH} 8,0.3 \mathrm{M} \mathrm{NaCl}, 10 \%$ glycerol, 0.6\% DBM, and $2 \mathrm{mM}$ DTT. SecA: wild-type SecA was purified as described [103] 
with the following modifications. Intact washed cells were incubated on ice for 30 minutes with $8 \mathrm{mM}$ EDTA to chelate $\mathrm{Mg}^{2+}$ in the cell envelope. The cells were pelleted and washed twice to remove the EDTA before being lysed by three cycles of freezing and thawing in the presence of lysozyme. The removal of EDTA before lysis is crucial to prevent the extraction of zinc from SecA. Following centrifugation, SecA was purified from the relevant supernatants by chromatography. SecB was grown as described [104]. The cells were disrupted using a French Pressure Cell $\left(5.5 \times 10^{7}\right.$ $\mathrm{Pa})$ and centrifuged for 2 hours at $65 \mathrm{k} \mathrm{rpm}(6800 \mathrm{rad} / \mathrm{s})$ using a Type $90 \mathrm{Ti}$ rotor (Beckman, Coulter). The supernatant was loaded onto a QAE column (TosoHaas) and $\mathrm{SecB}$ was purified with a gradient of $200-480 \mathrm{mM} \mathrm{NaCl}$ in $20 \mathrm{mM}$ TrisCl, $2 \mathrm{mM}$ DTT, pH 7. SecB was eluted around $350 \mathrm{mM} \mathrm{NaCl}$. Concentrations of the proteins were determined spectrophotometrically at $280 \mathrm{~nm}$ using coefficients of extinction as follows: SecB tetramer $47600 \mathrm{M}^{-1} \mathrm{~cm}^{-1}$; SecA monomer $78900 \mathrm{M}^{-1} \mathrm{~cm}^{-1}$; SecYEG $45590 \mathrm{M}^{-1} \mathrm{~cm}^{-1}$.

\section{D.2 Preparation of radiolabeled precursors}

pOmpA and pGBP were produced in strain MM52 from plasmids pAL612 and pAL725 carrying the ompA gene or the $m g l B$ gene, respectively. Cells were grown in M9 minimal media supplemented with 19 amino acids (no Leu) and were radiolabeled by addition of ${ }^{14} \mathrm{C}$-Leu (PerkinElmer) [46].

\section{D.3 Preparation of proteoliposomes}

Lipids (E. coli polar lipid extract, Avanti) in chloroform were blown dry with $\mathrm{N}_{2}$ and placed in a vacuum chamber overnight. A dry mechanical vacuum pump (XDS5, Edwards) was used to prevent back streaming of oil, a potential contaminant. 
Dried lipids were suspended in 10 mM HEPES, pH 7.6, 30 mM KAc, 1 mM MgAc 2 . Unilamellar liposomes were prepared by extrusion through membranes $(\sim 100 \mathrm{~nm}$ pore diameter, LiposoFast, Avestin). To form proteoliposomes, the liposomes were swelled, but not disrupted, using a ratio of detergent to lipids of $4.65 \mathrm{mM}$ DBM to $5 \mathrm{mM}$ lipids [105]. After swelling for 3 hours at room temperature, the proteins to be incorporated were added: SecYEG at $5 \mu \mathrm{M}$ (the molar ratio of SecYEG to lipid was 1:1000) and for coassembly of SecA (YEG. A), SecA at $5 \mu \mathrm{M}$ dimer. Incubation was continued for 1 hour at room temperature followed by addition of BioBeads SM-2 (BioRad) to remove the detergent. The proteoliposomes were isolated by centrifugation at 436 000g, 20 minutes at $4{ }^{\circ} \mathrm{C}$ in a TL100.1 rotor (Beckman). The pellet was suspended in the same buffer and centrifuged again as earlier. The final pellet was suspended to give a concentration of $\sim 10 \mathrm{mM}$ lipid and $10 \mu \mathrm{M}$ SecY. The suspension was stored at $-80{ }^{\circ} \mathrm{C}$.

\section{D.4 Translocation assay in solution}

A time course of translocation assay was performed before each AFM experiment using the precursors containing disulfide-stabilized loops. Translocation of 1 $\mu \mathrm{M}$ oxidized pOmpA or pGBP, labeled with a ${ }^{14} \mathrm{C}$-L-amino acid mixture, into proteoliposomes was carried out at $30{ }^{\circ} \mathrm{C}$ under conditions of limiting SecY $(1 \mu \mathrm{M})$ with SecA $(1.2 \mu \mathrm{M}$ dimer $)$, SecB $(1 \mu \mathrm{M}$ tetramer $)$, and EGTA $(1 \mathrm{mM})$ to prevent pGBP from folding, as described [73], with the following modifications: A volume of sample selected for translocation quantification was subjected to proteinase $\mathrm{K}$ digestion. The radioactivity in the protein bands corresponding to protected precursors obtained from electrophoresis was measured using a Fujifilm FLA 3000 phosphorimager in the linear range of its response. Figure 66 depicts the precursor proteins protected from digestion during the proteinase $\mathrm{K}$ treatment. From each time point, intensity of the 
protected precursor band (everything included in the right curly bracket) was normalized with the intensity of the reference band (everything included in the left curly bracket). Thus \% translocation was carried out. With known volume and concentration of solution subjected to electrophoresis, mol precursor protected was calculated.

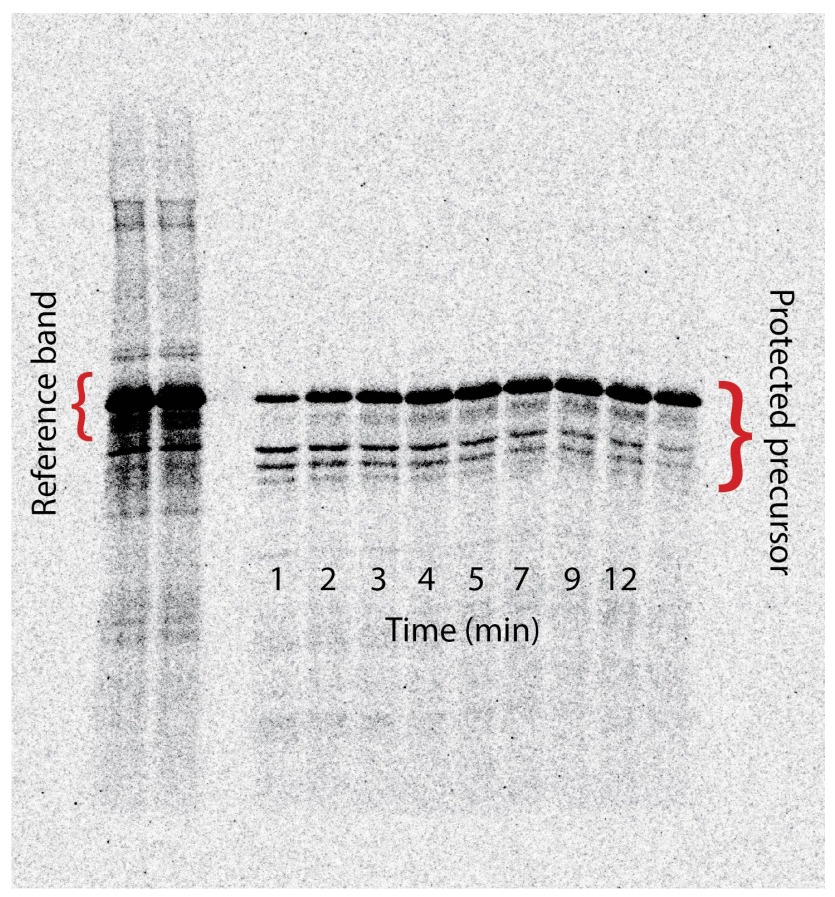

Figure 66: Quantification of translocation in solution

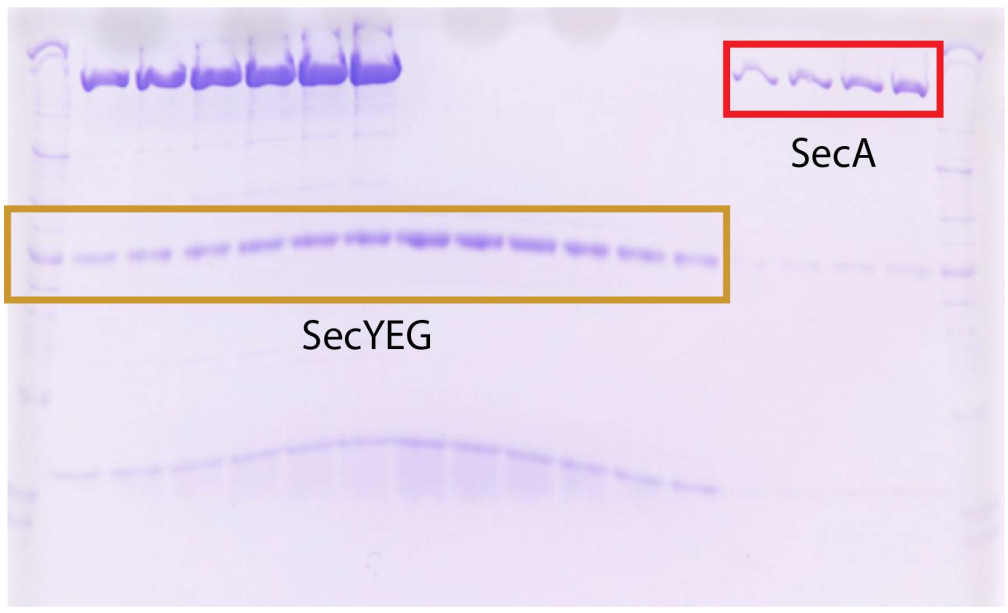

Figure 67: Quantification of proteins (SecY and SecA) in proteoliposome 
Each batch of proteoliposome was quantified after coassemble. The proteoliposome solution was prepared at several dilutions and subjected to electrophoresis. The intensity of each band from different dilutions was quantified using ImageQuant software.

\section{D.5 Liposome preparation}

\section{Experimental procedure}

1. Distribute E. coli polar lipid extract in chloroform to culture tubes such that there is approximately $13 \mathrm{mg}$ lipid in each tube.

2. Evaporate chloroform using Ar gas under the hood, forming a thin film of lipid on the wall of the tube. Keep rotating the tube while drying to evenly form the film.

3. Place tube in the dry vacuum chamber and pump overnight.

Note: A dry mechanical vacuum pump is used here to prevent back-streaming of oil, a potential contaminant.

4. For storage, backfill tubes with Ar gas, seal with parafilm, and store at $-20^{\circ} \mathrm{C}$.

5. Take one tube of dry lipid prepared above (or out from the $-20^{\circ} \mathrm{C}$ freezer).

6. Add $550 \mu \mathrm{L}$ of buffer containing $10 \mathrm{mM}$ HEPES, pH 7.6, $30 \mathrm{mM} \mathrm{KAc}, 1 \mathrm{mM}$ $\mathrm{MgAc}_{2}$ to the tube.

7. Let sit at room temperature for 1 hour to swell the lipid.

8. Use pipet to rinse the wall of the tube and hydrate all lipids.

9. Wet a $200 \mathrm{~nm}$ membrane and filter supports with buffer. 
10. Insert membrane into lipid extruder with filter supports on each side. The extrusion parts are shown in Figure 68

11. Label one side of the extruder with "IN".

12. Load a $1000 \mu \mathrm{L}$ Hamilton syringe with lipid suspension.

13. Insert syringe into the extruder side labeled "IN".

14. Insert an empty $1000 \mu \mathrm{L}$ Hamilton syringe to the other side of the extruder.

15. Pass the solution through the extruder 20-30 times (or until the solution becomes visually clear).

16. Remove solution from the side opposite "IN" into Eppendorf tubes.

17. Quantify lipid concentration using an appropriate assay as described in D.6.

18. Store at $-80^{\circ} \mathrm{C}$

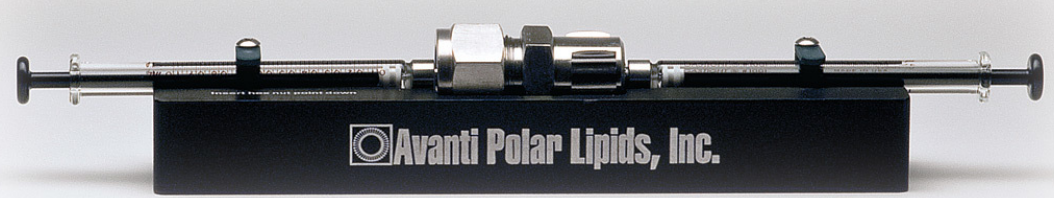




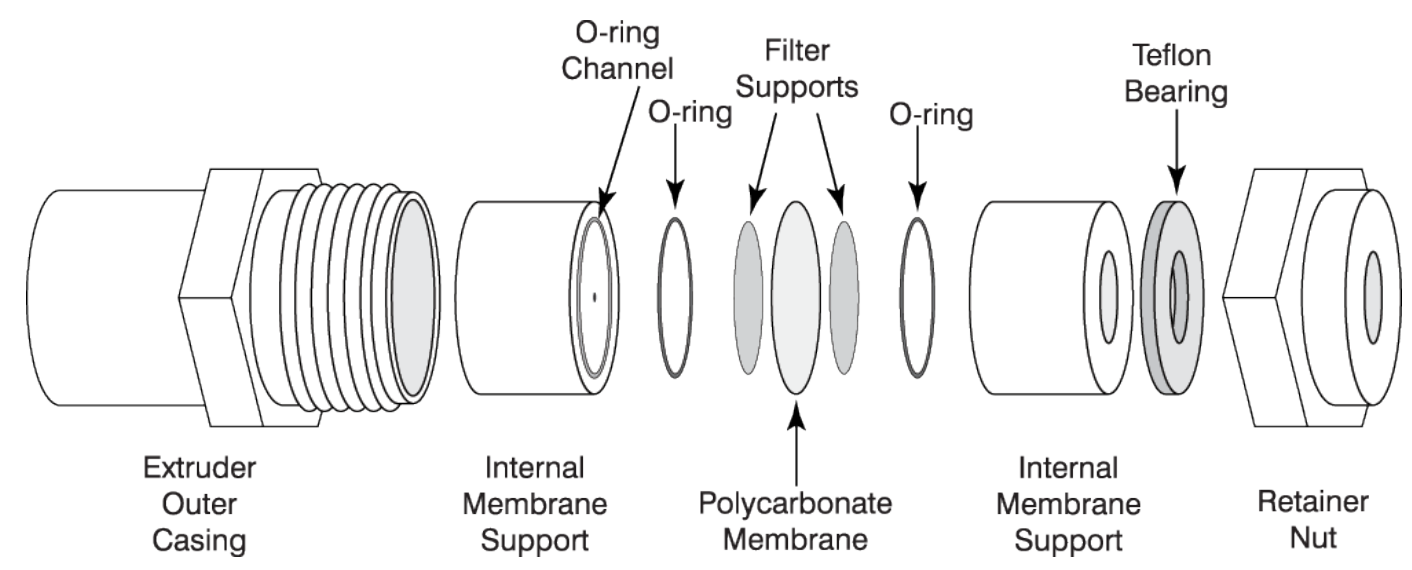

Figure 68: Avanti polar lipids extruder and the parts

\section{D.6 Quantification of lipid in liposome}

To quantify the concentration of lipid in liposome, we use a colorimetry method to measure the amount of phosphorous, the headgroup of liposomes, by stripping it off the head group of the phosphor lipid bilayer in the liposome. A mixture of sulfuric acid, ascorbic acid, hydrogen peroxide and ammonium molybdate was used to extract the phosphorous and form a complex that turned blue. The intensity of the blue color correlated to the amount of phosphorous in the solution.

\section{Experimental procedure}

1. Prepare $2.5 \mathrm{~mL}$ of $9 \mathrm{~N} \mathrm{H}_{2} \mathrm{SO}_{4}, 5 \mathrm{~mL}$ of $10 \%$ ascorbic acid ( $1 \mathrm{~g}$ in $10 \mathrm{~mL} \mathrm{H} \mathrm{H}_{2} \mathrm{O}$ ), $5 \mathrm{~mL}$ of $2.5 \%$ ammonium molybdate tetrahydrate $\left(0.63 \mathrm{~g}\right.$ in $\left.25 \mathrm{~mL} \mathrm{H} \mathrm{H}_{2} \mathrm{O}\right), 1 \mathrm{~mL}$ of $30 \% \mathrm{H}_{2} \mathrm{O}_{2}\left(3 \mathrm{~mL} \mathrm{H} \mathrm{H}_{2} \mathrm{O}_{2}\right.$ and $\left.7 \mathrm{~mL} \mathrm{H} \mathrm{H}_{2} \mathrm{O}\right)$, and $600 \mu \mathrm{L}$ of $0.65 \mathrm{mM}$ standard phosphorous solution.

2. In glass test tube, prepare standard phosphorous solution in 6 dilutions, lipid sample in 3 dilutions, and 1 blank solution as shown in table 4 . 
Table 4: Total phosphorous assay for liposome quantification

\begin{tabular}{|c|c|c|c|}
\hline Tube \# & Sample & Volume $(\mu \mathrm{L})$ & $\lambda(820 \mathrm{~nm})$ \\
\hline 1 & Blank & 0 & \\
\hline 2 & Standard 1 & 25 & \\
\hline 3 & Standard 2 & 50 & \\
\hline 4 & Standard 3 & 75 & \\
\hline 5 & Standard 4 & 100 & \\
\hline 6 & Standard 5 & 125 & \\
\hline 7 & Standard 6 & 150 & \\
\hline 8 & Lipid Sample 1 & 50 & \\
\hline 9 & Lipid Sample 2 & 100 & \\
\hline 10 & Lipid Sample 3 & 150 & \\
\hline
\end{tabular}

3. Add 2 boiling stones in each tube to prevent superheating.

4. Add $225 \mu \mathrm{L}$ of $10 \mathrm{~N} \mathrm{H}_{2} \mathrm{SO}_{4}$ to each tube.

5. Place the tubes in the heat block at $220^{\circ} \mathrm{C}$ for 25 minutes.

Note: This step is to release the phosphorous of the phospholipid bilayer.

6. Take the tubes off heat block and let cool at room temperature for 5 minutes.

7. Add $75 \mu \mathrm{L}$ of $\mathrm{H}_{2} \mathrm{O}_{2}$ to the bottom of each tube.

8. Place the tubes back to the heat block at $220{ }^{\circ} \mathrm{C}$ for another 30 minutes

9. During this step, preheat the water bath to $100{ }^{\circ} \mathrm{C}$.

10. Take the tubes off the heat block and let cool to room temperature.

11. Add $1.95 \mathrm{~mL} \mathrm{H}_{2} \mathrm{O}, 0.25 \mathrm{~mL}$ ammomium molybdate, and $0.25 \mathrm{~mL}$ ascorbic acid to each tube. 
12. Mix well using vortex.

13. Place all tubes in the rack and incubate 10 minutes in the water bath at 100 ${ }^{\circ} \mathrm{C}$ with marble cap on every tube for 10 minutes.

14. Take the tubes from the water bath and let cool to room temperature before taking absorption measurement.

15. Measure the absorptions at $820 \mathrm{~nm}$.

16. The slopes of the standard curve and the sample curve are used to calculate the phosphorous concentration of the liposome. The example of the standard and the sample curves are shown in Figure 69a and 69b. Knowing that there is one phosphorous in the phosphate head group of the lipid bilayer, the concentration of phosphorous is thus the concentration of the lipid in the liposome solution.
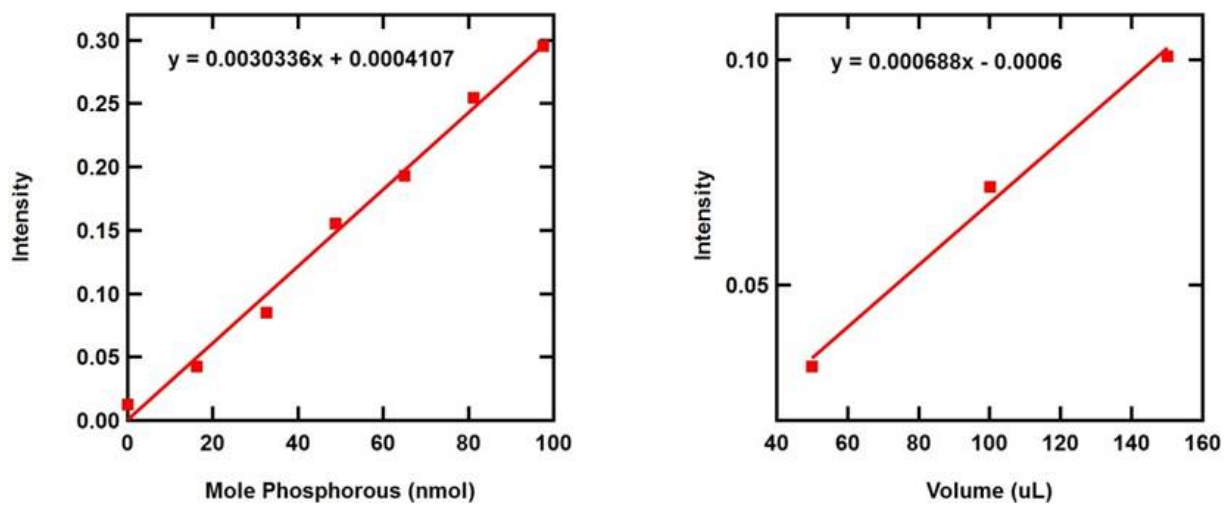

Figure 69: The standard curve is on the left and the sample is on the right. The ratio of the slope of the sample and the slope of the standard curve allows calculation of the concentration of the liposome sample.

\section{D.7 Taussky-Shorr assay}

Taussky-Shorr assay is another chemical assay used to measure the amount of inorganic phosphate product similar to lipid quantification. This assay uses sulfuric 
acid, ammonium molybdate, and ferrous sulfate to extract phosphate and form complex that gives color. This assay does not involve heat, and thus it is more suitable for measuring inorganic phosphate product produced by protein in an enzymatic reaction such as dephosphorization reaction and pyrophosphatase enzyme [106].

\section{Experimental procedure}

1. In an Eppendorf tube A, dissolve $5 \mathrm{mg}$ of ammonium molybdate in $50 \mu \mathrm{L}$ of 10 $\mathrm{N} \mathrm{H}_{2} \mathrm{SO}_{4}$, and $400 \mu \mathrm{L} \mathrm{H}_{2} \mathrm{O}$.

2. In an Eppendorf tube B, dissolve $25 \mathrm{mg}$ of Ferrous (II) sulfate in $450 \mu \mathrm{L} \mathrm{H}_{2} \mathrm{O}$.

3. In 6 separate Eppendorf tubes, add 6 different dilutions of standard phosphate solution $(0.65 \mathrm{mM})$ as shown in table 5 .

Table 5: Taussky-Shorr assay for phosphate product quantification

\begin{tabular}{|c|c|c|c|}
\hline Tube \# & Sample & Volume $(\mu \mathrm{L})$ & $\lambda(660 \mathrm{~nm})$ \\
\hline 1 & Blank & 0 & \\
\hline 2 & Standard 1 & 10 & \\
\hline 3 & Standard 2 & 15 & \\
\hline 4 & Standard 3 & 20 & \\
\hline 5 & Standard 4 & 25 & \\
\hline 6 & Standard 5 & 30 & \\
\hline 7 & Standard 6 & 35 & \\
\hline 8 & Sample 1 & 10 & \\
\hline 9 & Sample 2 & 20 & \\
\hline 10 & Sample 3 & 30 & \\
\hline
\end{tabular}

4. Mix solution in Eppendorf tube A and B then vortex to ensure mixing. This solution is called color mix. 
Note: The color mixture should be freshly made on the day of the experiment.

5. Add $35 \mu \mathrm{L}$ of the color mix to each tube. Use vortex to mix.

6. Measure absorption of the solution at $660 \mathrm{~nm}$

7. Convert volume of the solution to concentration and plot absorbance as a function of solution concentration. Plot two separate curves: one for standards and another one for samples.

8. Take the ratio of the slope of the standard curve and the sample curve to calculate the concentration of the samples. 


\section{References}

[1] Baró, A. M. and Reifenberger, R. G. Atomic force microscopy in liquid: biological applications. John Wiley \& Sons, (2012).

[2] Berg, J. M., Tymoczko, J. L., and Stryer, L. Biochemistry. W.H. Freeman, New York, 7th ed edition, (2012).

[3] Dufrêne, Y. F., Ando, T., Garcia, R., Alsteens, D., Martinez-Martin, D., Engel, A., Gerber, C., and Müller, D. J. Nature Nanotechnology 12(4), 295-307 April (2017).

[4] Shaw, G. A. Measurement Science and Technology 29(7), 072001 July (2018).

[5] El Kirat, K., Morandat, S., and Dufrêne, Y. F. Biochimica et Biophysica Acta (BBA) - Biomembranes 1798(4), 750-765 April (2010).

[6] Gari, R. R. S., Frey, N. C., Mao, C., Randall, L. L., and King, G. M. Journal of Biological Chemistry 288(23), 16848-16854 (2013).

[7] Mao, C., Cheadle, C. E., Hardy, S. J. S., Lilly, A. A., Suo, Y., Gari, R. R. S., King, G. M., and Randall, L. L. Proceedings of the National Academy of Sciences 110(29), 11815-11820 July (2013).

[8] Chada, N., Sigdel, K. P., Gari, R. R. S., Matin, T. R., Randall, L. L., and King, G. M. Scientific Reports 5, 12550 July (2015).

[9] Radmacher, M., Fritz, M., Hansma, H. G., and Hansma, P. K. Science 265(5178), 1577-1579 September (1994).

[10] Edwards, D. T., Faulk, J. K., Sanders, A. W., Bull, M. S., Walder, R., LeBlanc, M.-A., Sousa, M. C., and Perkins, T. T. Nano letters 15(10), 7091-7098 (2015). 
[11] Edwards, D. T. and Perkins, T. T. Journal of Structural Biology 197(1), 13-25 January (2017).

[12] Howard, J. and Clark, R. Applied Mechanics Reviews 55(2), B39-B39 March (2002).

[13] French, A. P. Vibrations and Waves. CRC Press, December (2017).

[14] Crowell, B. Fullerton, California (2015).

[15] Sigdel, K. P., Pittman, A. E., Matin, T. R., and King, G. M. In Nanoscale Imaging: Methods and Protocols, Lyubchenko, Y. L., editor, Methods in Molecular Biology, 49-62. Springer, New York, NY (2018).

[16] Edwards, D. T., Faulk, J. K., LeBlanc, M.-A., and Perkins, T. T. Biophysical journal 113(12), 2595-2600 (2017).

[17] Müller, D. J., Fotiadis, D., Scheuring, S., Müller, S. A., and Engel, A. Biophysical Journal 76(2), 1101-1111 February (1999).

[18] Yu, H., Siewny, M. G. W., Edwards, D. T., Sanders, A. W., and Perkins, T. T. Science 355(6328), 945-950 March (2017).

[19] Pelz, B., Žoldák, G., Zeller, F., Zacharias, M., and Rief, M. Nature Communications 7, 10848 February (2016).

[20] Butt, H.-J., Cappella, B., and Kappl, M. Surface Science Reports 59(1), 1-152 October (2005).

[21] Cooper, G. M. The Cell: A Molecular Approach. 2nd edition (2000).

[22] Radzicka, A. and Wolfenden, R. Science 267(5194), 90-93 January (1995). 
[23] Ramanathan, A., Savol, A., Burger, V., Chennubhotla, C. S., and Agarwal, P. K. Accounts of Chemical Research 47(1), 149-156 January (2014).

[24] Chada, N., Chattrakun, K., Marsh, B. P., Mao, C., Bariya, P., and King, G. M. Science Advances 4(10), eaat8797 October (2018).

[25] Chada, N. Watching biological nanomotors at work : insights from singlemolecule studies. Thesis, University of Missouri-Columbia, (2017).

[26] Tsirigotaki, A., De Geyter, J., Šoštaric, N., Economou, A., and Karamanou, S. Nature Reviews Microbiology 15(1), 21 (2017).

[27] Crane, J. M. and Randall, L. L. EcoSal Plus 7(2) (2017).

[28] Randall, L. L. and Hardy, S. J. S. Cell 46(6), 921-928 September (1986).

[29] Cunningham, K., Lill, R., Crooke, E., Rice, M., Moore, K., Wickner, W., and Oliver, D. The EMBO Journal 8(3), 955-959 March (1989).

[30] Dalal, K., Chan, C. S., Sligar, S. G., and Duong, F. Proceedings of the National Academy of Sciences 109(11), 4104-4109 March (2012).

[31] Matin, T. R., Sigdel, K. P., Utjesanovic, M., Marsh, B. P., Gallazzi, F., Smith, V. F., Kosztin, I., and King, G. M. Langmuir 33(16), 4057-4065 April (2017).

[32] Keramisanou, D., Biris, N., Gelis, I., Sianidis, G., Karamanou, S., Economou, A., and Kalodimos, C. G. Nature Structural \& Molecular Biology 13(7), 594602 July (2006).

[33] Zimmer, J., Nam, Y., and Rapoport, T. A. Nature 455(7215), 936-943 October (2008). 
[34] Gouridis, G., Karamanou, S., Sardis, M. F., Schärer, M. A., Capitani, G., and Economou, A. Molecular Cell 52(5), 655-666 December (2013).

[35] Allen, W. J., Corey, R. A., Oatley, P., Sessions, R. B., Baldwin, S. A., Radford, S. E., Tuma, R., and Collinson, I. eLife 5 May (2016).

[36] Bauer, B. W., Shemesh, T., Chen, Y., and Rapoport, T. A. Cell 157(6), 14161429 June (2014).

[37] Kedrov, A., Kusters, I., and Driessen, A. J. M. Biochemistry 52(39), 6740-6754 October (2013).

[38] Koch, S., Wit, J. G. d., Vos, I., Birkner, J. P., Gordiichuk, P., Herrmann, A., Oijen, A. M. v., and Driessen, A. J. M. Journal of Biological Chemistry 291(43), 22534-22543 October (2016).

[39] Fessl, T., Watkins, D., Oatley, P., Allen, W. J., Corey, R. A., Horne, J., Baldwin, S. A., Radford, S. E., Collinson, I., and Tuma, R. eLife 7, e35112 June (2018).

[40] Catipovic, M. A., Bauer, B. W., Loparo, J. J., and Rapoport, T. A. The EMBO Journal 38(9), e101140 May (2019).

[41] Gari, R. R. S., Chattrakun, K., Marsh, B. P., Mao, C., Chada, N., Randall, L. L., and King, G. M. Science Advances 5(6), eaav9404 June (2019).

[42] Vandenberk, N., Karamanou, S., Portaliou, A. G., Zorzini, V., Hofkens, J., Hendrix, J., and Economou, A. Structure 27(1), 90-101.e6 January (2019).

[43] Seinen, A.-B. and Driessen, A. J. Annual Review of Biophysics 48(1), 185-207 (2019). 
[44] Serdiuk, T., Steudle, A., Mari, S. A., Manioglu, S., Kaback, H. R., Kuhn, A., and Müller, D. J. Science Advances 5(1), eaau6824 January (2019).

[45] Young, J. and Duong, F. Journal of Biological Chemistry 294(10), 3577-3587 (2019).

[46] Bariya, P. and Randall, L. L. Journal of Bacteriology, JB.00493-18 October (2018).

[47] Oliver, D. Journal of bacteriology 201(1), e00618-18 (2019).

[48] Bippes, C. A. and Muller, D. J. Reports on Progress in Physics 74(8), 086601 July (2011).

[49] Tamm, L. K. and McConnell, H. M. Biophysical Journal 47(1), 105-113 January (1985).

[50] Castellana, E. T. and Cremer, P. S. Surface Science Reports 61(10), 429-444 November (2006).

[51] Karner, A., Nimmervoll, B., Plochberger, B., Klotzsch, E., Horner, A., Knyazev, D. G., Kuttner, R., Winkler, K., Winter, L., Siligan, C., Ollinger, N., Pohl, P., and Preiner, J. Nature Nanotechnology 12(3), 260-266 March (2017).

[52] Glazier, R. and Salaita, K. Biochimica et Biophysica Acta (BBA) - Biomembranes 1859(9, Part A), 1465-1482 September (2017).

[53] Perkins, T. T. Laser $\&$ Photonics Reviews 3(1-2), 203-220 (2009).

[54] Bezanilla, M., Drake, B., Nudler, E., Kashlev, M., Hansma, P. K., and Hansma, H. G. Biophysical Journal 67(6), 2454-2459 December (1994). 
[55] Viani, M. B., Pietrasanta, L. I., Thompson, J. B., Chand, A., Gebeshuber, I. C., Kindt, J. H., Richter, M., Hansma, H. G., and Hansma, P. K. Nature Structural \& Molecular Biology 7(8), 644-647 August (2000).

[56] Nakanishi, K., Sakiyama, T., and Imamura, K. Journal of Bioscience and Bioengineering 91(3), 233-244 January (2001).

[57] Kranz, C., Kueng, A., Lugstein, A., Bertagnolli, E., and Mizaikoff, B. Ultramicroscopy 100(3), 127-134 August (2004).

[58] Schaap, I. A. T., Carrasco, C., de Pablo, P. J., and Schmidt, C. F. Biophysical Journal 100(10), 2450-2456 May (2011).

[59] Kodera, N., Yamamoto, D., Ishikawa, R., and Ando, T. Nature 468(7320), 72 (2010).

[60] Ando, T., Uchihashi, T., and Scheuring, S. Chemical Reviews 114(6), 31203188 March (2014).

[61] Arredondo, M., Stoytcheva, M., Morales-Reyes, I., and Batina, N. Biotechnology 83 Biotechnological Equipment 0(0), 1-10 May (2018).

[62] Nievergelt, A. P., Banterle, N., Andany, S. H., Gönczy, P., and Fantner, G. E. Nature Nanotechnology 13(8), 696-701 August (2018).

[63] Mao, C., Hardy, S. J. S., and Randall, L. L. Journal of Bacteriology 191(3), 978-984 February (2009).

[64] Schulz, J. C., Warr, G. G., Butler, P. D., and Hamilton, W. Physical Review E 63(4), 041604 (2001). 
[65] Majkrzak, C. F., Carpenter, E., Heinrich, F., and Berk, N. F. Journal of Applied Physics 110(10), 102212 (2011).

[66] Majkrzak, C., O’Donovan, K., and Berk, N. In Neutron Scattering from Magnetic Materials, 397-471. Elsevier (2006).

[67] Hoogerheide, D. P., Heinrich, F., Maranville, B. B., and Majkrzak, C. F. Journal of Applied Crystallography 53(1) (2020).

[68] Chattrakun, K., Schaefer, K. G., Chandler, L. S., Marsh, B. P., and King, G. M. Methods in Molecular Biology (in press).

[69] Chattrakun, K., Hoogerheide, D. P., Mao, C., Randall, L. L., and King, G. M. Langmuir 35(37), 12246-12256 (2019).

[70] Lill, R., Dowhan, W., and Wickner, W. Cell 60(2), 271-280 (1990).

[71] Robson, A., Gold, V. A. M., Hodson, S., Clarke, A. R., and Collinson, I. Proceedings of the National Academy of Sciences 106(13), 5111-5116 March (2009).

[72] Economou, A. and Wickner, W. Cell 78(5), 835-843 September (1994).

[73] Brundage, L., Hendrick, J. P., Schiebel, E., Driessen, A. J. M., and Wickner, W. Cell 62(4), 649-657 August (1990).

[74] Shekhar, P., Nanda, H., Lösche, M., and Heinrich, F. Journal of Applied Physics 110(10), 102216 November (2011).

[75] Majkrzak, C. F., Metting, C., Maranville, B. B., Dura, J. A., Satija, S., Udovic, T., and Berk, N. F. Physical Review A 89(3), 033851 March (2014). 
[76] Carrion-Vazquez, M., Oberhauser, A. F., Fowler, S. B., Marszalek, P. E., Broedel, S. E., Clarke, J., and Fernandez, J. M. Proceedings of the National Academy of Sciences 96(7), 3694-3699 March (1999).

[77] Kohn, J. E., Millett, I. S., Jacob, J., Zagrovic, B., Dillon, T. M., Cingel, N., Dothager, R. S., Seifert, S., Thiyagarajan, P., Sosnick, T. R., Hasan, M. Z., Pande, V. S., Ruczinski, I., Doniach, S., and Plaxco, K. W. Proceedings of the National Academy of Sciences 101(34), 12491-12496 August (2004).

[78] Cremer, P. S. and Boxer, S. G. The Journal of Physical Chemistry B 103(13), 2554-2559 April (1999).

[79] Marsh, B. P., Chada, N., Gari, R. R. S., Sigdel, K. P., and King, G. M. Scientific Reports 8(1), 1-12 January (2018).

[80] Woodbury, R. L., Hardy, S. J. S., and Randall, L. L. Protein Science 11(4), 875-882 April (2002).

[81] Fak, J. J., Itkin, A., Ciobanu, D. D., Lin, E. C., Song, X.-J., Chou, Y.-T., Gierasch, L. M., and Hunt, J. F. Biochemistry 43(23), 7307-7327 June (2004).

[82] Schiebel, E., Driessen, A. J., Hartl, F.-U., and Wickner, W. Cell 64(5), 927-939 (1991).

[83] de Keyzer, J., van der Does, C., Kloosterman, T. G., and Driessen, A. J. Journal of Biological Chemistry 278(32), 29581-29586 (2003).

[84] Erlandson, K. J., Or, E., Osborne, A. R., and Rapoport, T. A. Journal of Biological Chemistry 283(23), 15709-15715 June (2008).

[85] Müller, D. and Engel, A. Biophysical journal 73(3), 1633-1644 (1997). 
[86] Rief, M., Gautel, M., Oesterhelt, F., Fernandez, J. M., and Gaub, H. E. Science 276(5315), 1109-1112 May (1997).

[87] Rief, M., Oesterhelt, F., Heymann, B., and Gaub, H. E. Science 275(5304), 1295-1297 February (1997).

[88] Findik, B. T., Smith, V. F., and Randall, L. L. Protein Science 27(3), 681-691 March (2018).

[89] Mori, H. and Ito, K. Journal of Biological Chemistry 281(47), 36249-36256 (2006).

[90] Utjesanovic, M., Matin, T. R., Sigdel, K. P., King, G. M., and Kosztin, I. Scientific Reports 9(1), 1-11 January (2019).

[91] Matin, T. R., Utjesanovic, M., Sigdel, K. P., Smith, V. F., Kosztin, I., and King, G. M. Langmuir 36(8), 2143-2152 (2020).

[92] Churnside, A. B. and Perkins, T. T. FEBS Letters 588(19), 3621-3630 October (2014).

[93] Ahn, S., Milner, A. J., Fütterer, K., Konopka, M., Ilias, M., Young, T. W., and White, S. A. Journal of Molecular Biology 313(4), 797-811 November (2001).

[94] Parfenyev, A. N., Salminen, A., Halonen, P., Hachimori, A., Baykov, A. A., and Lahti, R. Journal of Biological Chemistry 276(27), 24511-24518 July (2001).

[95] Riedel, C., Gabizon, R., Wilson, C. A. M., Hamadani, K., Tsekouras, K., Marqusee, S., Pressé, S., and Bustamante, C. Nature 517(7533), 227-230 January (2015). 
[96] Chen, B., Doucleff, M., Wemmer, D. E., De Carlo, S., Huang, H. H., Nogales, E., Hoover, T. R., Kondrashkina, E., Guo, L., and Nixon, B. T. Structure 15(4), 429-440 (2007).

[97] Nystrom, N. A., Levine, M. J., Roskies, R. Z., and Scott, J. R. In Proceedings of the 2015 XSEDE Conference: Scientific Advancements Enabled by Enhanced Cyberinfrastructure, 1-8, (2015).

[98] Towns, J., Cockerill, T., Dahan, M., Foster, I., Gaither, K., Grimshaw, A., Hazlewood, V., Lathrop, S., Lifka, D., Peterson, G. D., Roskies, R., Scott, J. R., and Wilkins-Diehr, N. Computing in Science Engineering 16(5), 62-74 September (2014).

[99] Vrugt, J. A., ter Braak, C., Diks, C., Robinson, B. A., Hyman, J. M., and Higdon, D. International Journal of Nonlinear Sciences and Numerical Simulation 10(3) January (2009).

[100] Kienzle, P. A., Krycka, J., Patel, N., Metting, C., Sahin, I., Fu, Z., Chen, W., Mont, A., and Tighe, D. (2016).

[101] Miroux, B. and Walker, J. E. Journal of molecular biology 260(3), 289-298 (1996).

[102] Cannon, K. S., Or, E., Clemons, W. M., Shibata, Y., and Rapoport, T. A. The Journal of Cell Biology 169(2), 219-225 April (2005).

[103] Randall, L. L., Crane, J. M., Lilly, A. A., Liu, G., Mao, C., Patel, C. N., and Hardy, S. J. S. Journal of Molecular Biology 348(2), 479-489 April (2005). 
[104] Randall, L. L., Topping, T. B., Smith, V. F., Diamond, D. L., and Hardy, S. J. S. In Methods in Enzymology, volume 290 of Molecular Chaperones, 444-459. Academic Press January (1998).

[105] Lambert, O., Levy, D., Ranck, J.-L., Leblanc, G., and Rigaud, J.-L. Biophysical Journal 74(2), 918-930 February (1998).

[106] Taussky, H. H. and Shorr, E. Journal of Biological Chemistry 202(2), 675-685 (1953). 


\section{VITA}

Kanokporn Chattrakun was born in Phichit, a small town approximately $300 \mathrm{~km}$ north of Bangkok, Thailand. She received her Bachelor of Science degree in Physics from Mahidol University, Thailand. She ventured to the United States and obtained her Master of Science degree in Physics from the University of Texas at El Paso. She, later, earned her Doctor of Philosophy in Physics in May of 2020 working with Dr. Gavin King investigating biological systems at a single molecule level utilizing traditional biochemical activity assays and atomic force microscopy. After finishing her PhD, she will join St. Jude Children's research hospital in Memphis, Tennessee as a part of its Structural Biology department. 